Walter Martins Rodrigues

Antônio Ronaldo Gomes Garcia

\title{
COLETÂNEA DE ARTIGOS DE MATEMÁTICA EM \\ PERSPECTIVA DE APLICAÇÃo
}
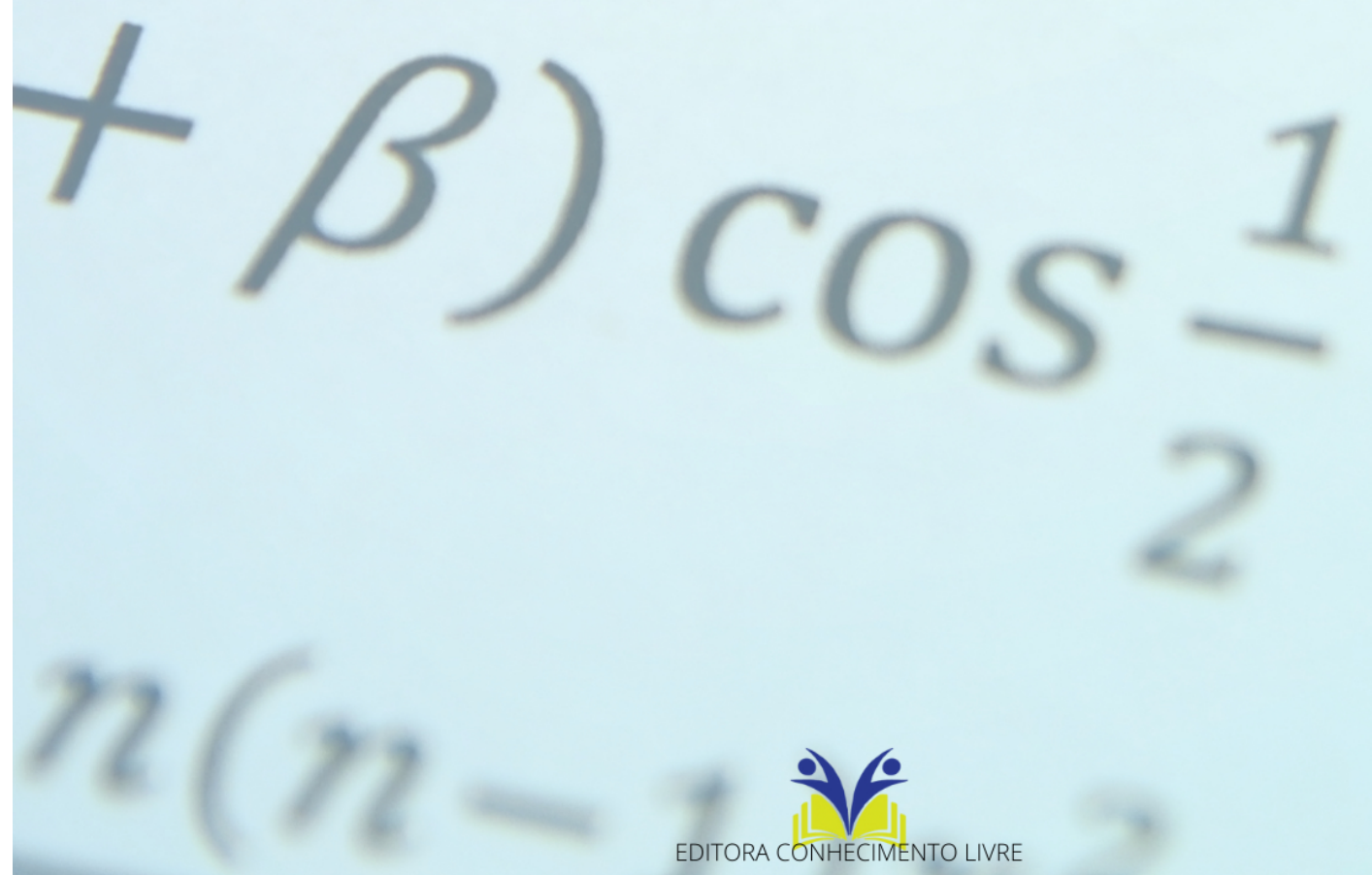
Walter Martins Rodrigues

Antônio Ronaldo Gomes Garcia

Coletânea de artigos de matemática em perspectiva de aplicação

$1^{\text {a }}$ ed.

Piracanjuba-GO

Editora Conhecimento Livre

Piracanjuba-GO 
$1^{\mathrm{a}} \mathrm{ed}$

\section{Dados Internacionais de Catalogação na Publicação (CIP)}

Rodrigues, Walter Martins

R146C Coletânea de artigos de matemática em perspectiva de aplicação

/ Walter Martins Rodrigues. Antônio Ronaldo Gomes Garcia. - Piracanjuba-GO

Editora Conhecimento Livre, 2021

114 f.: il

DOI: $10.37423 / 2021$. edcl301

ISBN: 978-65-89955-79-5

Modo de acesso: World Wide Web

Incluir Bibliografia

1. modelo-matemático 2. ciência-e-realidade 3. novos-modelos-matemáticos 4. simulaçõesmatemáticas 5. inovação-na-abordagem-matemática I. Rodrigues, Walter Martins II. Garcia, Antônio Ronaldo Gomes III. Título

https://doi.org/10.37423/2021.edc1301

O conteúdo dos artigos e sua correção ortográfica são de responsabilidade exclusiva dos seus respectivos autores. 


\title{
EDITORA CONHECIMENTO LIVRE
}

\section{Corpo Editorial}

\author{
Dr. João Luís Ribeiro Ulhôa \\ Dra. Eyde Cristianne Saraiva-Bonatto \\ Dr. Anderson Reis de Sousa \\ MSc. Frederico Celestino Barbosa \\ MSc. Carlos Eduardo de Oliveira Gontijo
}

$\underline{\text { MSc. Plínio Ferreira Pires }}$

Editora Conhecimento Livre

Piracanjuba-GO 


\section{SUMÁRIO}

CAPÍTULO 1 5 MODELO MATEMÁTICO DE ELEMENTOS DE CONTROLE EPIDEMIOLÓGICO DA COVID-19

Walter Martins Rodrigues

Antonio Ronaldo Gomes Garcia

Jennifer do Vale Silva

Roberto Pequeno de Souza

Janilson Pinheiro de Assis

Paulo César Linhares da Silva

DOI 10.37423/210704514

\section{CAPÍTULO 2}

SIMULAÇÃO DE BULBO MOLHADO ISOLADO E FAIXA MOLHADA NA IRRIGAÇÃO POR

GOTEJAMENTO SUPERFICIAL

Luiz Ailton de Araújo Souza

Sérgio Luiz Aguilar Levien

Walter Martins Rodrigues

DOI 10.37423/210704529

\section{CAPÍTULO 3}

ANÁLISE DAS PROPRIEDADES ELETRÔNICAS EM FIOS E PONTOS QUÂNTICOS

SEMICONDUTORES DE SI/SIGE

Ricardo Hugo Nunes Medeiros Filho

DOI 10.37423/210804564

CAPÍTULO 4

ÁLGEBRAS ESTANDARMENTE ESTRATIFICADAS: ÁLGEBRAS DE NAKAYAMA

Fidel Hernández Advíncula

DOI 10.37423/210804576

CAPÍTULO 5

ESTABILIZAÇÃO POR REALIMENTAÇÃO DE SAÍDA NO POSICIONAMENTO REGIONAL DE PÓLOS

Elmer Rolando Llanos Villarreal

Walter Martins Rodriuges

Samara Jessica Monte Bezerra

Jose Alfredo Ruiz Vargas

DOI 10.37423/210804626

CAPÍTULO 6

STRATEGIES AND INFECTIOUS DISEASES

ORLANDO STANLEY JURIAANS

DOI 10.37423/210904780 


\section{Capítulo 1}

d.)

\section{MODELO MATEMÁTICO DE ELEMENTOS DE CONTROLE EPIDEMIOLÓGICO DA COVID-19}

Walter Martins Rodrigues

Antonio Ronaldo Gomes Garcia

Jennifer do Vale Silva

Roberto Pequeno de Souza

Janilson Pinheiro de Assis

Paulo César Linhares da Silva
Universidade Federal Rural do Semi-Árido

Universidade Federal Rural do Semi-Árido

Universidade Federal Rural do Semi-Árido

Universidade Federal Rural do Semi-Árido

Universidade Federal Rural do Semi-Árido

Universidade Federal Rural do Semi-Árido 
Resumo: Para a modelagem de doenças infecciosas, normalmente a população é separada em classes, nossa estratégia é trabalhar apenas com as classes: Suscetíveis, Infectados e Recuperados. Na classe de suscetíveis, consideramos os indivíduos sem infecção e que ainda não tiveram contato com o vírus. Para a Covid-19, estamos supondo que o indivíduo infectado, após se curar, fica imune para aquele vírus que já foi infectado. Nestas condições propomos um modelo para a Covid-19, que tem parâmetros mais específicos associados ao comportamento epidemiológico do transmissor, podendo refletir melhor o aspecto mais local da dinâmica de contaminação, permitindo um controle mais preciso do acompanhamento da doença. 


\section{INTRODUÇÃO:}

Os modelos matemáticos em estudos epidemiológicos são ferramentas essenciais para uma compreensão mais precisa do padrão de uma epidemia na sociedade. Hoje esses problemas são modelados a partir de equações diferenciais que descrevem um equilíbrio dinâmico evidenciado pela ação da doença. Nesse sistema de equações diferenciais que representa o problema, Hamer (1906) foi o primeiro a propor um modelo que considerava que o número de casos novos de infecção pela a doença estava relacionado ao produto entre as densidades de indivíduos suscetíveis e infectados veja [6]. Em 1927 Kernack e McKendrick propuseram e resolveram um teorema fundamental para área de modelagem epidemiológica, descreveram o fenômeno limiar, este evidencia que para se ter um surto epidêmico a densidade de indivíduos suscetíveis é superior a certo valor crítico.

Os modelos matemáticos clássicos nessa área de pesquisa são os modelos determinísticos e os modelos estocásticos. Os modelos determinísticos são importantes para tratar da dinâmica de grandes populações. Em tais modelos os parâmetros de entrada (taxa de morte pela doença, taxa de recuperação etc) são fixas, assim as predições ao longo do tempo são predeterminadas pela entrada inicial de parâmetros. Estes modelos são obtidos a partir de equações diferenciais que descrevem a dinâmica do processo. Em nosso caso vamos trabalhar com um modelo deterministico de tipo de estrutura SIR, em que o compartimento $S$ representa a classe dos indivíduos suscetívieis, I representa a classe dos indivíduos infectados e $R$ é associado à classe dos indivíduos recuperados ou removidos, seja por adquirirem imunidade ou por morte em consequência da doença. McKendrick está entre os pioneiros da modelagem estocástica de fenômenos epidemiológicos. Este tipo de modelo permite que o número de indivíduos que se deslocam entre os compartimentos tenha variação aleatória. Desta forma estes modelos oferecem maior facilidade de tratar sobre a heteregeneidade da doença. Estes modelos são obtidos à partir de equações diferenciais parciais. Tais modelos são mais usados quando se trata de modelagem envolvendo pequenas populações.

A modelagem matemática de um processo epidemiológico como a Covid-19, está relacionada a alguns aspectos teóricos fundamentais. Nesse sentido os modelos matemáticos são caracterizados por permitirem uma descrição robusta, flexiva, precisa e dispõe de capacidade preditiva confiável. A robustez de um modelo matemático é descrito pela capacidade do modelo em reproduzir os dados observados e capturar a dinâmica da doença ao longo prazo. A flexibilidade do modelo matemático está relacionada a capacidade de adaptação a outros fenômenos similares e a rápida assimilação de novas observações. Já a precisão é dada no sentido de convergência numérica das soluções do 
problema. Em particular, para os modelos epidemiolóogicos espera-se que a solução convirja assintoticamente para o equilíbrio.

Por outro lado, a capacidade preditiva do modelo consiste na obtenção de estimativas futuras que são consistentes com a realidade. Outro fator que estamos relevando é a impossibilidade de reinfecção, ou seja consideramos que um indivíduo suscetível poderá ser infectado no máximo uma vez.

\section{2)Parâmetros epidemiológicos}

1) A força de uma infecção é definida como a densidade de incidência da doença e é representada por $\lambda$. Este parâmetro pode ser obtido a partir de dados epidemiológicos sob a hipótese que no estado inicial da epidemia o número de indivíduos infectados cresce aproximadamente a uma taxa constante, tal taxa é chamada de taxa de crescimento da epidemia. Matematicamente temos:

$$
\frac{d I}{d t}=\lambda \Longleftrightarrow I(t)=I(0) \cdot e^{\lambda} \rightarrow \lambda
$$

Portanto, temos que $t \lambda=\ln \left(\frac{I(t)}{I(0)}\right)=\ln (I(t))-\ln (I(0)) \Rightarrow \lambda=\frac{\ln (I(t))-\ln (I(0))}{t}$.

Uma forma simples de determinar o parâmetro da força de infecção_ é a partir do ajuste de uma reta de regressão aos valores dos números de casos acumulados da doença.

2) O número de reprodutibilidade basal é o parâmetro correspondente ao número de médio de infectados secundários causados por um indivíduo de uma população de suscetíveis durante o tempo em que ele é infectante. Este parâmetro epidemiológico é representado por $R_{0}$. Assim o parâmetro $R_{0}$ caracteriza a habilidade de um organismo infeccioso invadir uma população totalmente suscetível e estabelecer-se nela durante o período que estiver infeccioso.

Quando a doença tem as características de se transitir somente na forma direta e com dependência a certa faixa etária o parâmetro $R_{0}$ pode ser obtido através da força de infecção $\lambda$. De acordo com [6], analiticamente e no aspecto epidemiológico esses dois limiares relacionam-se. Considerando $L$ a expectativa média de vida da população, nesse caso o parâmetro $R_{0}$ é dado por $R_{0}=1+\lambda L$, detalhes a respeito dessa expressão estão em [6].

Dependendo da complexidade do modelo matemático pode se obter $R_{0}$ diretamente das equações do modelo. Considere o modelo tipo SIR proposto por Kernack e McKendrick, veja [6], com dinâmica vital e governado pelas equações 


$$
\begin{array}{cc}
\frac{d S}{d t}= & \mu N-\beta \frac{S I}{N}-\mu S \\
\frac{d I}{d t}= & \beta \frac{S I}{N}-(\gamma+\mu) I \\
\frac{d R}{d t}= & \gamma I-\mu R
\end{array}
$$

Considerando a população constante durante o processo, temos $N=S+I+R$, o parâmetro $\mu$ representa a taxa de nascimento e de morte natural, $\gamma$ representa a taxa per capita de recuperação da infecção da Covid-19 e $\beta$ corresponde ao número de contatos (decisivos, críticos) entre suscetíveis S e infectados I, aqui considera-se contato decisivo aqueles que resultam infecção pelo Corona vírus. Suponhamos que o período de infeç̧ão é descrito aproximadamente por $\frac{1}{\gamma+\mu}$ dias. De acordo com [9] temos que $R_{0}=\frac{\beta}{\gamma+\mu}$. Para o modelo acima a dedução do valor de $R_{0}$

é direta. Quando o modelo for mais complexo a expressão que define $R_{0}$ torna-se mais complicada. Uma formalização matemática mais geral de $R_{0}$ foi feita por Dublin e Lotka (1925) e Kuczinski (1928). Nesse contexto $R_{0}$ é a taxa líquida de reprodução da doença por fase, veja [4]

$$
R_{0}=\int_{0}^{\infty} b(x) F(x) d x
$$

Onde $F(x)=e^{-\int_{0}^{x} \mu(h) d h}$ corresponde a função de sobrevivência, note que tal função está relacionada com a mortalidade da idade específica $\mu(h)$. Ou seja, corresponde a probabilidade de um indivíduo recém-nascido sobreviver pelo menor até a idade $x$, e $b(x)$ é o número médio de descendentes que um indivíduo produzirá por unidade de tempo até a idade $x$.

3) Obtenção de dados para um mapeamento mais preciso

Os dados determinantes dos parâmetros e variáveis do modelo devem ser obtidos de forma amostral, assim sendo obtidos via uma Pesquisa de mensuração de dados. Esses dados sendo Levantados de forma amostral regional, junto aos principais ambientes contaminantes, às pessoas da área em estudo, levando em conta amostragem relacionadas aos aspectos econômicos, de saúde individual e sociais. Os seguintes aspectos devem ser considerados:

a) O gênero das pessoas;

b) Faixas etárias: crianças, adolescentes, aduntos jovem e idosos. Grupos de idades:0-8, 9-15, 16$17,18-24,25-29,30-39,40-49,50-69$ e 70 anos ou mais 
c) Instrução: nível fundamental, Ensino Médio, Superior;

d) Principais comorbidades: diabétes, tuberculose, obesidade, asma, pressão alta;

e) Porte do município (em número de habitantes): Até 20 mil, Mais de 20 a 100 mil, e com mais de 100 mil habitantes;

f) Condição de moradia (espaço, saneamento), densidade demográfica

Trabalhar com intervalo de confiança estimado de 95\% e a margem de erro máxima estimada, para que a significância da representação dos dados seja boa. O modelo matemático proposto, leva em conta que existe uma difusão hierárquica do vírus, aspectos geográficos, demográficos e sociais são considerados. Percebe-se que a difusão do vírus ocorre, no Brasil, indo de cidades maiores para menores. Para levarmos em conta aspectos locais, em consonância com o tamanho da população, mas também a influência econômica na macrorregião e os particulares aspectos sociais das localidades em foco de estudo.

\section{4) Aspectos operacionais}

Suposição inicial: população da região permanece constante durante o processo.

$N_{h}$ : população humana da região $\Omega$;

$N_{v}$ : população de pessoas circulantes de $\Omega$ sem imunidade ao coronavírus;

$S_{h}$ : população humana região $\Omega$ suscetível ao coronavírus;

$I_{h}$ : população de $\Omega$ formado pelas pessoas que estão infectadas pelo coronavirus;

$R_{h}$ : população de $\Omega$ formado pelas pessoas recuperadas de uma infecção pelo coronavirus;

$S_{v}=N_{v} \cup I_{h}$ : pessoas com potencial de difusão do coronavírus;

$I_{v}=N_{v}-I_{g}$ : pessoas que promovem a difusão de pontos contaminadas pelo coronavírus, onde $I_{g}$ indica população humana infectada em total isolamento.

Considere a taxa de variação de $N_{v}$ em relação ao tempo:

$$
\frac{d N_{v}}{d t}=A(t)-\mu_{v} N_{v}
$$

Sendo $A(t)$ a taxa de variação de $I_{h}$ em relação ao tempo, conhecida como taxa de renovação de potenciais difusores. O parâmetro $\mu_{v}$ é a taxa de variação de $I_{g}$ em relação ao tempo. Em alguma circunstância é importante considerar $A(t)$ constante. Observe que se $A$ é decrescente se preponderar 
as variáveis favoráveis ao melhor controle (mais informação, mais obediência às recomendações dos especialistas, ação média com está eficiente etc).

Observe que no caso em $A(t)$ é constante, temos que:

$$
\lim _{t \rightarrow \infty} N_{v}(t)=\frac{A}{\mu_{v}}
$$

Considere $\mu_{h}$ : o parâmetro que corresponde a taxa de nascimentos e mortes de humanos no período do evento; o parâmetro $\gamma_{h}$ a taxa de humanos que superaram a doença. A passagem das pessoas da categoria de suscetíveis para o conjunto de infectados, depende em essência de vários fatores como: número médio de pontos de infecção deixados por uma pessoa que é tocado por alguém por dia, a probabilidade de transmissão. Bem como o número de suscetíveis e infectados.

Seja $m$ o parâmetro correspondente ao número de pontos de acumulação em que o coronavirus mantém ativo por mais de um dia. Assim a probabilidade de que um ponto de contaminação atinja uma pessoa do ambiente $\Omega$ é dado por $\frac{N_{h}}{m+N_{h}}$.

Ademais, uma pessoa tem contato com a quantidade $\frac{b N_{v} N_{h}}{\left(m+N_{h}\right) N_{h}}$ pontos de contaminação por dia, uma pessoa contaminada, em média, cerca de $\frac{b N_{h}}{\left(m+N_{h}\right)}$ contatos com outras pessoas. Portanto temos a validade da fórmula: Probabilidade de transmissibilidade de uma pessoa ou ponto infectante durador para uma pessoa é

$$
\beta_{h} \frac{b N_{v} N_{h}}{N_{h}\left(N_{h}+m\right)} \frac{I_{v}}{N_{v}}=\frac{\beta_{h} b I_{v}}{N_{h}+m}
$$

Aqui $\beta_{h}$ é o número médio de suscetíveis infectados por um indivíduo por unidade de tempo. A probabilidade de uma pessoa ser contaminada e gerar novo contaminante, ou ponto de acumulação de contágio

$$
\frac{\beta_{h} b N_{h}}{N_{h}+m} \frac{I_{h}}{N_{h}}=\frac{\beta_{v} b I_{h}}{N_{h}+m}
$$

Nestas condições o sistema de equações diferenciais que modelam o problema de expansão da Covid19 é dada por: 


$$
\begin{array}{cc}
\frac{d \overline{S_{h}}}{d t}= & \mu_{h}\left(n_{h}-\overline{S_{h}}\right)-\frac{\beta_{h} b \overline{S_{h}}}{N_{h}+m} \overline{I_{v}} \\
\frac{d I_{v}}{d t}= & \frac{\beta_{h} b S_{h}}{N_{h}+m} I_{v}-\left(\mu_{h}+\gamma_{h}\right) I_{h} \\
\frac{d \bar{R}_{h}}{d t}= & \gamma_{h} I_{h}-\mu_{h} R_{h} \\
\frac{d \overline{S_{v}}}{d t}= & A-\frac{\beta_{h} b S_{h}}{N_{h}+m} I_{h}-\mu_{v} S_{v} \\
\frac{d I_{v}}{d t}= & \frac{\beta_{h} b S_{h}}{N_{h}+m} I_{h}-\mu_{v} I_{v}
\end{array}
$$

Sendo $\gamma$ a taxa de recuperação per capita da infecção. A população humana do problema deve permanecer constante. Temos que $N_{h}=S_{h}+I_{h}+R_{h}$ e $N_{v}=S_{v}+I_{v}$.

O sistema de equações referido é invariante diante destas expressões de $N_{h}$ e $N_{v}$, dado que qualquer solução de tal sistema de equações, no caso de $A$ ser constante, temos:

$$
\frac{N_{h}}{d t}=0 \mathrm{e} \frac{N_{v}}{d t}=\frac{d}{d t}\left(\frac{A}{\mu_{v}}\right)=0
$$

Dado que $N_{h}$ é constante e $N_{v}$ tende a $\frac{A}{\mu_{v}}$ pontualmente quando $t$ tende a $\infty$. Todas as soluções do sistema satisfazem as expressões canônicas de $N_{h}$ e $N_{v}$. Podemos trabalhar com as proporções referentes as populações:

$$
S_{h}=\frac{\overline{S_{h}}}{N_{h}} \quad I_{h}=\frac{\overline{I_{h}}}{I_{h}} \quad S_{v}=\frac{\overline{S_{v}}}{N_{v}} \quad I_{v}=\frac{\overline{I_{v}}}{I_{v}}
$$

Desta forma temos $R_{h}=1-S_{h}-I_{h}$ e $S_{v}=1-I_{v}$. Obtemos o seguinte conjunto de três equações que descrevem a modelagem do problema

$$
\begin{aligned}
& \frac{d S_{h}}{d t}=\mu_{h}\left(I-S_{h}\right)-\beta_{h} b I_{v} \frac{A}{\mu_{v}\left(N_{h}+m\right)} \\
& \frac{d U_{h}}{d t}=\beta_{h} b I_{v} \frac{A}{\mu_{v}\left(N_{h}+m\right)}-\left(\gamma_{h}+\mu_{h}\right) I_{h} \\
& \frac{d I_{h}}{d t}=\beta_{h} b I_{h} \frac{A}{\mu_{v}\left(N_{h}+m\right)}-\mu_{v} I_{v}
\end{aligned}
$$

Nestas condições a reprodutibilidade basal é determinada, de acordo com [1] pode-se estimar o parâmetro limiar $R_{0}$ do modelo, baseado em [3] é descrito a explicitamente a expressão para $R_{0}$ :

$$
R_{0}=\sqrt{\frac{b^{2} \beta_{v} \beta_{h} A}{\left(N_{h}+m\right)^{2} \mu_{v}^{2} N_{h}\left(\mu_{h}+\gamma_{h}\right)}}=\frac{b}{\mu_{v}\left(N_{h}+m\right)} \sqrt{\frac{\beta_{v} \beta_{h} A}{\mu_{h}+\gamma_{h}}}
$$

Em [1] mostra-se que usar a estimativa quadrada de $R_{0}$ não alteram o desenvolvimento do modelo, assim pode trabalhar com a estimativa de 


$$
R_{0}=\frac{A \beta_{v} \beta_{h} b^{2}}{\mu_{v}^{2}\left(\mu_{h}+\gamma_{h}\right) N_{h}\left(m+N_{h}\right)^{2}}
$$

O equilíbrio endêmico da doença é o tipo de estado de equilíbrio em que a doença se estabelece e permanece atingindo a população. No caso da covid-19, quando $R_{0}>1$,

Pode-se mostrar que o equilíbrio endêmico $E=\left(S_{h}^{*}, I_{h}^{*}, I_{v}^{*}\right)$ é dado por:

$$
S_{h}^{*}=\frac{\beta+M}{\beta+R_{0} . M} \quad S_{h}^{*}=\frac{R_{0}-1}{\beta-h_{0} . M} \quad S_{h}^{*}=\frac{\beta\left(R_{0}-1\right)}{H_{0}(\beta+M)}
$$

Onde

$$
M=\frac{\gamma_{h}}{\mu_{h}}+1, \beta=\frac{b \beta_{h} N_{h}}{\mu_{v}\left(N_{h}+m\right)} \text { e } R_{0}=\frac{b^{2} \beta_{h} \beta_{h} A}{\left(N_{h}+m\right)^{2} \mu_{2}^{2} N_{h}\left(\mu_{h}+\gamma_{h}\right)} .
$$

4.2) Estabilidade do modelo:

Vamos fazer a análise de estabilidade do sistema de equações 1 . Sejam $E_{1}$ e $E_{2}$ os pontos de equilíbrio de 1, a estabilidade assintóptica dos equilíbrios indica que as soluções iniciando suficientemente próximo dos equílibrios permanecem próximas e tendem a estes equilíbrios quando o tempo $t$ tende ao término do processo epidêmico $t_{F}$. Por outro lado, a instabilidade do equilíbrio indica que as soluções começando arbitrariamente próximos destes pontos de equilíbrio não necessariamente se aproxima dele ao longo do tempo.

Como o modelo considerado é referente a um processo biológico as soluções estáveis adquirem uma importância essencial no estudo do modelo. Por razões biológicas, apenas soluções não negativas do problema de valor inicial são aceitáveis. Mais precisamente, é necessário estudar as propriedades da solução do sistema de equações diferenciais associado ao modelo, sujeito a determinadas condições iniciais no conjunto fechado. $O$ estudo da estabilidade será realizado em 3 fases:

1. Estabilidade local do equilíbrio livre de doença e do equilíbrio endêmico de acordo com o critério de Routh-Hurwitz;

2. estabilidade global livre de doença, com uso da função de Liuponov e o o princípio da invariância de Lesalle

3. Estabilidade global do equilíbrio endêmico.

a) Inicialmente analisa-se a estabilidade local dos pontos de equilíbrio:

$$
E_{1}=(1,0,0) \text { e } D f\left(E_{1}\right)
$$


A derivada do campo vetorial associado ao sistema e aplicado em $E_{1}$, assim

$$
D f\left(E_{1}\right)=\left[\begin{array}{ccc}
\mu_{h} & 0 & \frac{-b \beta_{h} A}{\mu_{v}\left(N_{h}+m\right)} \\
0 & -\gamma_{h}-\mu_{h} & \frac{b \beta_{h} A}{\mu_{v}\left(N_{h}+m\right)} \\
0 & \frac{b \beta_{v} N_{h}}{N_{h}+m} & -\mu_{v}
\end{array}\right]
$$

O polinômio característico de $D f\left(E_{1}\right)$ é:

$$
p(\lambda)=\left(\mu_{h}+h\right) \cdot\left[-\left(\gamma_{h}+\mu_{h}+\mu_{v}\right)\left(\mu_{v}+\lambda\right)+\frac{b^{2} \beta_{v} \beta_{h} A N_{h}}{\mu_{v}\left(N_{h}+m\right)^{2}}\right]
$$

Note que $\lambda=-\mu_{h}$ é raiz de $p(\lambda)$. Pelo critério de Routh-Hurwitz, todas as raízes de $p(\lambda)$ ou são negativas, ou possuem parte real i, se $R_{0}<1$. Assim, para $R_{0}<1$ temos que $E_{1}$ é o equilíbrio livre de doença, que é assintoticamente estável.

Se $R_{0}>1$, o ponto de equilíbrio $E_{1}$ torna se instável hiperbólico, enquanto $E_{2}$, o ponto de equilíbrio endêmico, torna-se ponto de estabilidade.

Para tratarmos da estabilidade global, vamos usar a mesma estratégia de [8], essa estabilidade é importante no sentido de que esta independe dos tamanhos das subpopulações de cada, as soluções do modelo matemático converge para o equilíbrio.

Estabilidade global de $E_{1}$ : Considere a função de Lyapunov $V(t)$ :

$$
V(t)=\frac{b \beta_{h} A I_{v}(t)}{\mu_{v}^{2}\left(N_{h}+m\right)}+I_{h}(t)
$$

Claro que $V(t) \geq 0$ para todo $t$. Vamos aplicar o princípio da invariância de Lisalle para concluir que se $R_{0}<1$, então $E_{1}$ é globalmente assintoticamente estável.

Observação: Princípio da invariância de Lesalle: Se $x^{*}$ é ponto de equilíbrio do sistema $x^{\prime}=f(x)$, seja $U \subset R^{n}$ um conjunto aberto contendo $x^{*}$ e $L: U \rightarrow R$ a função de Lyapunov para $x^{*}$, seja $P$ uma vizinhança fechada de $x^{*}$. Se $P$ é positivamente invariante e que não existe solução em $P-\left\{x^{*}\right\}$ para qual $L$ é constante. Nessas condições $x^{*}$ é assintoticamente estável e $P$ está contido na região de atraçao de $x^{*}$. A demonstração deste resultado pode ser encontrada em [8]. 
Calculando $\frac{d V}{d t}$ e igualando a zero, obtém $Z(V)$ o conjunto dos pontos críticos de $V$, claro que $Z(V)$ é finito, e pode se dado pela equações:

$$
\begin{aligned}
& \left(1-S_{h}\right) I_{v}=0, \quad I_{h}=0 \text { se } R_{0}<1, \text { e } \\
& \left(1-S_{h}\right) I_{v}=0, \quad I_{v} \cdot I_{h}=0 \text { se } R_{0}=1
\end{aligned}
$$

Analisando o sistema (1), temos que o maior conjunto compacto invariante é:

$$
\left\{\left(S_{h}, I_{h}, I_{v}\right): \frac{d V}{d t}=0\right\}=\left\{E_{1}\right\}
$$

Assim pelo princípio de Lesalle temos que $E_{1}$ é globalmente assintoticamente estável se $R_{0} \leq 1$.

Vamos analisar o equilíbrio endêmico no caso em que $R_{0}>1$, nesse caso mostraremos que $E_{2}$ é localmente estável, com o uso do critério de Routh-Hurwitz.Considere a matriz $\operatorname{Df}\left(E_{2}\right)$ sendo a linearização do sistema (1) em torno do equilíbrio endêmico $E_{2}$ :

$$
D f\left(E_{2}\right)=\left[\begin{array}{ccc}
\mu_{h} \frac{\beta+M R_{0}}{\beta+M} & 0 & \left.-\mu_{h} \frac{M R_{0}}{\beta_{h}} \frac{(\beta+M)}{\left(\beta+M R_{0}\right.}\right) \\
\frac{\mu_{h} M\left(R_{0}-1\right.}{\beta+M} & -\mu_{h} M & \frac{\mu_{h} M R_{0}}{\beta} \frac{(\beta+M)}{\left(\beta+M R_{0}\right)} \\
0 & \frac{\mu_{v} \beta}{R_{0}} \frac{\left(\beta+R_{0} M\right)}{(\beta+M)} & -\mu_{v} R_{0} \frac{\beta+M}{\beta+R_{0} M}
\end{array}\right]
$$

Onde

$$
M=\frac{\gamma_{h}}{\mu_{h}}+1, \beta=\frac{b \beta_{v} N_{h}}{\mu_{v}\left(N_{h}+m\right)} \text { e } R_{0}=\frac{b^{2} \beta_{v} \beta_{h} A}{\left(N_{h}+m\right)^{2} \mu_{v}^{2} N_{h}\left(\mu_{h}+\gamma_{h}\right)}
$$

Considere $q(\lambda)$ o polinômio característico associado a $D f\left(E_{2}\right)$. Temos que $q(\lambda)=a_{3} \lambda^{3}+a_{2} \lambda^{2}+$ $a_{1} \lambda+a_{0}$, onde $a_{3}=1, a_{0}=\mu_{h}^{2} \mu_{v} M\left(R_{0}-1\right)$,

$$
\begin{gathered}
a_{2}=\mu_{h}\left[\frac{\text { beta }+M R_{0}}{\beta+M}+M\right]+\mu_{v} R_{0} \frac{\beta+M}{\beta+R_{0} M} \\
a_{1}=\mu_{h}^{2} M \frac{\text { beta }+M R_{0}}{\beta+M} \mu_{v} \mu_{h} R_{0}+\frac{\left(R_{0}-1\right) \mu_{h} \mu_{v} \beta M}{\beta+M R_{0}}
\end{gathered}
$$

Pelo critério de Routh-Hurwitz temos que $E_{2}$ é localmente assintoticamente estável. Uma vez que todos os coeficients são estritamente positivos, $a_{i}>0$ para $i \in\{0,1,2,3\}$, 
Sempre que $R_{0}>1$, além disso todos os parâmetros do modelo são negativos. Verificando que $a_{1} a_{2}>a_{0} a_{3}$, podemos concluir que $R_{0}>1$, logo o ponto $E_{2}$ é localmente assintoticamente estável. Usando uma extensão do teorema de Poincaré-Bendixson feita por Hirsch em [7], com os mesmos argumentos podemos garantir que o equilíbrio endêmico $E_{2}$ é globalmente estável.

\section{5) DISCUSSÕES FINAIS}

A análise de sensibilidade nos diz a importância de cada parâmetro é a transmissão de doenças. Tais informações não são cruciais apenas para o design experimental, mas também para a assimilação de dados e redução de modelos não lineares complexos, parâmetros relacionados à elementos geograficos e de densidade populacional local, possuem relevante peso.

O estudo da Sensibilidade é comumente usados para determinar a robustez de previsões de modelo para valores de parâmetros, uma vez que geralmente há erros na coleta de dados e valores de parâmetros presumidos, desta forma o modelo mostrou ter essas propriedades de robustez. Bem como, foi possível identificar os parâmetros dos parâmetros na simulação do modelo, mostrou que as variáveis associadas a elementos locais, demográficos e sociais têm um alto impacto no $R_{0}$ e devem ser alvo de estratégias de intervenção. A implementação prática do modelo, demanda recursos para realizar medidas estatisticamente bem modeladas, dos parâmetros do modelo proposto, demanda um trabalho conjunto de epidemiologistas, agentes de saúde e pesquisadores da modelagem matemática. Em contra partida o modelo tem estrutura teórica confiável, dinamismo e flexibilidade. Podendo ser uma poderosa ferramenta de controle e de tomada de decisão no trabalho de enfrentamento ao coronavirus. 


\section{6) REFERÊNCIAS BIBLIOGRÁFICAS}

[1] Anderson, R.M, May, R.M Infectious diseases of humans: diseases and control, Oxford Press., 1991.

[2] Butler, G., Freedman, H.I., Waltman, P. Uniformly persistent system . Proceedings American Mathematic Society, 298 (2), pp 418-431, 2004.

[3] Diekmann, O., Heesterbeek, J.A.P., Roberts, M.G. On definition and computatio of the basic reproduction rate $R_{0}$ in models for infectious diseases in heterogeneous population. Journal of Mathematic Biology, 28(4) 365-382, 1990.

[4] Heesterbeek, J.A.P., Dietz, K. The concept of $R_{0}$ in epidemic theory. Statistica Neerlandica, 50 (1): 89-110, 1996.

[5] Gomes,L.L.F ,Thomé, R.C.A, Modelagem Matemática do Aedes aegypti com a utilizaşão de mecanismos de combate à Dengue, Proceeding Series of the Brazilian Society of Applied and Computational Mathematics, Vol. 3, N. 1, 2015.

[6] Hethcote, H.W., The matehematics of infections diseases. SIAM review 42(4), 599$653,2000$.

[7] Hirsch, M. W. Systems of differential equations that are competitve or cooperative, iv: structural stability $i$ n three-dimensional systems. SIAM, journal on mathematical analysis, 21(5): 1225-1234, 1990.

[8] Hirsch, M. W., Smale, S., Devaney, R. L. Differential equation, dynamical systems, and an introduction the Chaos. Acad. Press 2012.

[9] Keeling, M.J., Rohani, J. Modeling infectious diseases in human and animals . Princeton Univ. Press., 2008.

[10] Thomé R.C.A., Yang H. M. Esteva L.: control of Aedes aegypti mosquitoes by the sterile insect technique and insecticide. Mathematical Biosciences 223, 2010.

[11] Esteva, L., Cristobal, V., Analysis of a dengue disease transmission model Mathematical Biosciences, 150(2), 131-151,1998. 


\section{Capítulo 2}

d.)

\section{SIMULAÇÃO DE BULBO MOLHADO ISOLADO E FAIXA MOLHADA NA IRRIGAÇÃO POR GOTEJAMENTO SUPERFICIAL}

Luiz Ailton de Araújo Souza

Sérgio Luiz Aguilar Levien

Walter Martins Rodrigues
Instituto Federal de Educação, Ciência e Tecnologia do Rio Grande do Norte

Universidade Federal Rural do Semi-Árido

Universidade Federal Rural do Semi-Árido 
Resumo: Neste trabalho é apresentado um modelo desenvolvido para a simulação do movimento de água no solo sob irrigação por gotejamento superficial que utiliza o método dos volumes finitos para a resolução da equação diferencial parcial de escoamento de água em meios porosos. O fluxo de água no solo é tratado tridimensionalmente para a determinação do seu movimento nas fases de infiltração e redistribuição. O modelo permite a determinação da forma e das dimensões do bulbo molhado isolado, e também, através da consideração de condições de contorno adequadas, simular a formação de bulbos molhados sobrepostos e, por consequência, de uma faixa molhada ao longo da linha lateral. Pode-se acompanhar a evolução dos bulbos ao longo do tempo, mediante a fixação do espaçamento entre emissores e da vazão do gotejador, podendo-se, ao final, obter os resultados que melhor se adequem às características do solo. O modelo demonstra estar de acordo com a teoria quando aplicado a solos de diversas texturas, podendo ser aplicado no dimensionamento de instalações de irrigação.

Palavras-chave: Modelagem computacional. Equação de Richards. Movimento de água no solo. Volume de solo molhado. Irrigação localizada. 


\section{INTRODUÇÃO}

A irrigação por gotejamento compreende a aplicação de água em apenas uma fração da área cultivada, em alta frequência e baixo volume, mantendo o solo na zona radicular das plantas sob alto regime de umidade. A área de solo molhado exposto à atmosfera fica bem reduzida e, consequentemente, é menor a perda de água por evaporação direta do solo. A água aplicada por este sistema penetra no solo através de sua superfície e se redistribui formando um bulbo molhado, onde as raízes se desenvolvem.

Por oferecer diversas vantagens em relação aos demais sistemas de irrigação, como maior produtividade, preservação de um nível de umidade adequado no interior do bulbo, economia de água, adequação a diversos tipos de terreno e culturas, entre outros, a irrigação por gotejamento, desde o início de sua aplicação comercial (na década de sessenta em Israel), expandiu-se rapidamente pelo mundo.

Na irrigação por gotejamento, a água aplicada pelo emissor forma inicialmente na superfície do solo, em torno do gotejador, um pequeno charco por onde acontece a infiltração da água no solo. Com o passar do tempo, decresce o fluxo aumentando gradativamente o tamanho do charco, até que este se estabilize, momento em que temos um equilíbrio entre o fluxo vertical de água na superfície e a vazão do gotejador. No entanto, o fluxo de água no interior do solo, para este tipo de irrigação, é tridimensional, produzindo um bulbo molhado, cuja forma e tamanho dependem principalmente da vazão do gotejador, do tempo de aplicação de água e das características do solo.

Com o avanço dos recursos computacionais, a modelagem matemática surgiu como uma solução alternativa e poderosa na predição do movimento tridimensional de água no solo, podendo-se processar a simulação de modelos complexos em um espaço de tempo aceitável. Ao longo do tempo, diversos modelos foram propostos na tentativa de realizar esta predição.

Além da profundidade molhada e do raio molhado, a modelagem matemática do movimento de água no solo pode realizar a determinação de informações importantes nas fases de elaboração de projetos de irrigação e de manejo, tais como, determinação do padrão de umidade no interior do bulbo, raio do disco saturado, alcance da frente de molhamento após determinado período de redistribuição, frequência de irrigação, espaçamento entre gotejadores, entre outros. Portanto, a obtenção destas informações através dos modelos reduz o trabalho e os custos das pesquisas de campo. 
Para a resolução da equação diferencial parcial de escoamento de água em meio poroso, tem-se utilizado diversas técnicas numéricas hoje disponíveis, já que tal equação não pode ser resolvida analiticamente, devido a sua alta não linearidade. Essas técnicas consistem em discretizar a equação de escoamento em relação ao tempo e ao espaço, podendo tratar problemas de fluxo tridimensional com relativa facilidade. Dessa forma, os modelos matemáticos simulados por computador usando técnicas numéricas, podem apresentar-se como uma ferramenta útil para o planejamento da irrigação.

Assim, neste trabalho, é apresentado um modelo, desenvolvido para a simulação dos processos de formação de bulbos molhados isolados e bulbos molhados sobrepostos, ou seja, formação de faixa molhada, quando esta é aplicada sob a forma de irrigação por gotejamento superficial, usando o método dos volumes finitos para a resolução da equação diferencial parcial de escoamento de água em meios porosos. O modelo permite a obtenção de informações como dimensões do bulbo, padrão de umidade no interior do bulbo e dimensões da faixa molhada formada, quando for o caso.

\section{REFERENCIAL TEÓRICO}

Na irrigação por gotejamento, a água aplicada pelo emissor forma inicialmente na superfície do solo, em torno do gotejador, um pequeno charco por onde acontece a infiltração da água no solo. Com o passar do tempo, decresce o fluxo aumentando gradativamente o tamanho do charco, até que este se estabilize, momento em que se tem um equilíbrio entre o fluxo vertical de água na superfície e a vazão do gotejador (DASBERG; BRESLER, 1985). No entanto, o fluxo de água no interior do solo, para este tipo de irrigação, é tridimensional, produzindo um bulbo molhado, cuja forma e tamanho dependem principalmente da vazão do gotejador, do tempo de aplicação de água e das características do solo.

No interior do bulbo, a água aplicada se move em grande parte através do solo sob condições de fluxo não saturado. Desta forma, a distribuição de água e a forma do volume molhado podem ser preditas pelas leis físicas de movimento capilar da água no solo para um ponto emissor.

Ao longo do tempo, diversos modelos foram propostos na tentativa de realizar esta predição. Wooding (1968) propôs uma solução analítica da equação de escoamento considerando que o fluxo de água através de uma cavidade circular de dimensões fixas seguia o regime permanente. $\mathrm{O}$ caráter deste estudo se aproxima da irrigação por gotejamento, já que após a estabilização do charco, pode-se considerar que o fluxo nessa zona de entrada de água é praticamente estacionário. Outras soluções 
analíticas foram propostas por Raats (1971), Warrick (1974), Loyola e Prevedello (2003) e Cook et al. (2003). Uma solução semi-analítica foi obtida por Coelho e Or (1999), para distribuição bidimensional da umidade no bulbo molhado, usando a solução analítica de Warrick (1974). Em Brandt et al. (1971), Botrel (1988), Berger (1994) e Rivera (2004), encontram-se soluções numéricas para a predição do movimento de água no solo na irrigação por gotejamento.

Tabuada e Berger (1998) desenvolveram modelos para simular situações de irrigação por gotejadores isolados, tubos porosos ou perfurados, semelhante à condição de Brandt et al. (1971), e microaspersores, resolvidos através da discretização das equações de escoamento da água no solo, usando um esquema implícito.

Na prática, vários gotejadores podem ser instalados próximos uns dos outros, junto à planta, para possibilitar o suprimento da quantidade de água necessária, bem como proporcionar o umedecimento da área mínima da superfície do solo.

Se os gotejadores em uma linha lateral estiverem espaçados uns dos outros a uma distância que não permita que seus bulbos se encontrem, o que se vê superficialmente são discos umedecidos isolados. No entanto, por questões agronômicas, pode-se ter uma disposição em que o espaçamento reduzido entre os gotejadores permita que os bulbos venham a se tocar ou mesmo se sobrepor, formando uma faixa úmida tanto superficialmente como ao longo da profundidade do bulbo. Para Pizarro Cabello (1990), a sobreposição é definida como uma porcentagem do raio molhado do bulbo, devendo estar compreendido entre $15 \%$ e $30 \%$.

Para a resolução da equação diferencial parcial de escoamento de água em meio poroso, tem-se utilizado diversas técnicas numéricas hoje disponíveis, já que tal equação não pode ser resolvida analiticamente, devido a sua alta não linearidade. Essas técnicas consistem em discretizar a equação de escoamento em relação ao tempo e ao espaço, podendo tratar problemas de fluxo tridimensional com relativa facilidade.

Entre os métodos numéricos tradicionais está o método dos volumes finitos (MVF), que tem por característica realizar um balanço de conservação da propriedade em cada volume elementar (MALISKA, 2004). Ao utilizar o MVF, o domínio que se pretende estudar é subdividido numa série de volumes de controle (VC) de dimensões finitas de forma arbitrária. Dessa forma, as leis que regem os processos que se pretende simular são aplicadas diretamente sobre os VC. Estes VC são representados por pontos nodais situados em seu centro. 


\section{METODOLOGIA}

A modelagem do movimento de água no solo utilizada neste trabalho foi desenvolvida e validada por Souza (2009). A metodologia pode ser encontrada, mais detalhadamente, em seu trabalho, de forma que, neste texto, será abordado de forma reduzida, porém não incompleta, a metodologia da modelagem do movimento de água no solo utilizada.

Sendo o domínio de estudo de dimensões relativamente pequenas, utiliza-se o MVF aplicado sobre uma malha de volumes inteiros ao longo de todo o domínio. Utilizando-se uma formulação explícita, o domínio D é discretizado em VC com a forma de pequenos cubos, formando uma malha de elementos contíguos, denominados aqui de células. Cada célula é representada por um nó, definido no centroide do VC, representado pelo ponto P. Um esquema da definição do domínio e sua discretização em células é apresentado na Figura 1.

FIGURA 1 - Esquema do domínio dividido em volumes de controle; à direita, o ponto P localizado no centroide de cada volume de controle

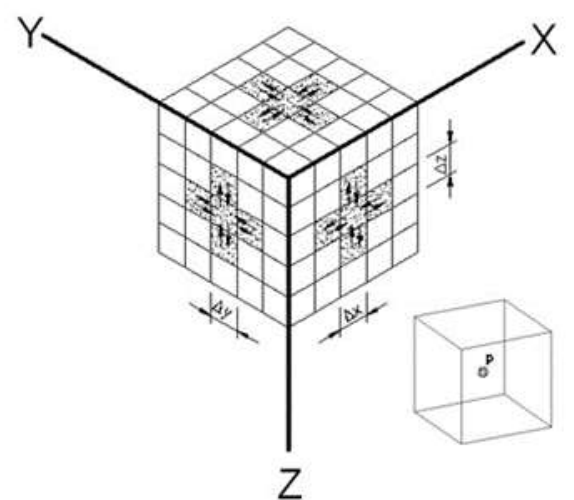

Fonte: Souza (2009)

A posição do emissor de água coincide com a origem dos eixos cartesianos $X, Y$ e $Z$. O eixo Z, perpendicular à superfície do solo, tem direção tomada como positiva no sentido do aumento da profundidade. Com vistas a reduzir o tempo computacional, o domínio é reduzido a um quarto do volume total. Cada volume de controle tem então comprimento, largura e altura definidos por $\Delta x, \Delta y$ e $\Delta z$, respectivamente, obtendo-se uma quantidade fixa de elementos em cada direção.

Considerando o solo um meio poroso estável, homogêneo e isotrópico, tem-se a equação de Richards da seguinte forma: 


$$
\frac{\partial \theta}{\partial t}=\frac{\partial}{\partial x}\left(K(\theta) \cdot \frac{\partial H}{\partial x}\right)+\frac{\partial}{\partial y}\left(K(\theta) \cdot \frac{\partial H}{\partial y}\right)+\frac{\partial}{\partial z}\left(K(\theta) \cdot \frac{\partial H}{\partial z}\right)
$$

Dada a discretização do domínio em volumes de controle e usando uma célula genérica de coordenadas (i, j, k), a equação (1) toma a seguinte forma:

$$
\frac{\Delta \theta}{\Delta \mathrm{t}}=\mathrm{K}_{\mathrm{MED}_{\mathrm{x}}}(\theta) \cdot \frac{\Delta\left(\Delta \mathrm{H}_{\mathrm{x}}\right)}{(\Delta \mathrm{x})^{2}}+\mathrm{K}_{\mathrm{MED}_{\mathrm{y}}}(\theta) \cdot \frac{\Delta\left(\Delta \mathrm{H}_{\mathrm{y}}\right)}{(\Delta \mathrm{y})^{2}}+\mathrm{K}_{\mathrm{MED}_{\mathrm{z}}}(\theta) \cdot \frac{\Delta\left(\Delta \mathrm{H}_{\mathrm{z}}\right)}{(\Delta \mathrm{z})^{2}}
$$

em que $\mathrm{K}_{\mathrm{MED}_{\mathrm{x}}}, \mathrm{K}_{\mathrm{MED}_{\mathrm{y}}}$ e $\mathrm{K}_{\mathrm{MED}_{z}}$ são as condutividades hidráulicas médias entre células adjacentes nas direções $X, Y$ e Z, respectivamente.

Como condição inicial, opta-se por considerar duas situações para o perfil hídrico inicial. Na primeira, o solo apresenta umidade volumétrica ou potencial matricial constante ao longo de todo o domínio. Na outra condição, o perfil hídrico inicial é resultante de uma irrigação anterior, após as fases de infiltração e redistribuição. Neste caso, cada célula do domínio tem seu próprio valor de umidade, aproximando o perfil de solo de uma situação mais realista.

Para a primeira situação, tem-se:

$$
\theta(i, j, k)=\theta_{0} ; \quad t=0 ; 0 \leq i \leq M-1 ; 0 \leq j \leq N-1 ; 0 \leq k \leq P-1
$$

Para a segunda situação, tem-se:

$$
\theta(i, j, k)=\theta_{0}(i, j, k) ; \quad t>0 ; 0 \leq i \leq M-1 ; 0 \leq j \leq N-1 ; 0 \leq k \leq P-1
$$

onde $\theta_{0}$ é a umidade inicial do solo em cada célula. $O$ tempo t com valor superior a zero, na equação (4), indica que a umidade inicial é proveniente de uma irrigação anterior e, portanto, não devendo ser tratado, para fins práticos, como o instante inicial.

Em relação às condições de contorno, o domínio é um prisma retangular de seis faces, os quais constituem as suas fronteiras, conforme apresentado na Figura 2. 
FIGURA 2 - Prisma retangular mostrando as fronteiras do domínio reduzido, considerando-se o gotejador posicionado no ponto $\mathrm{A}$

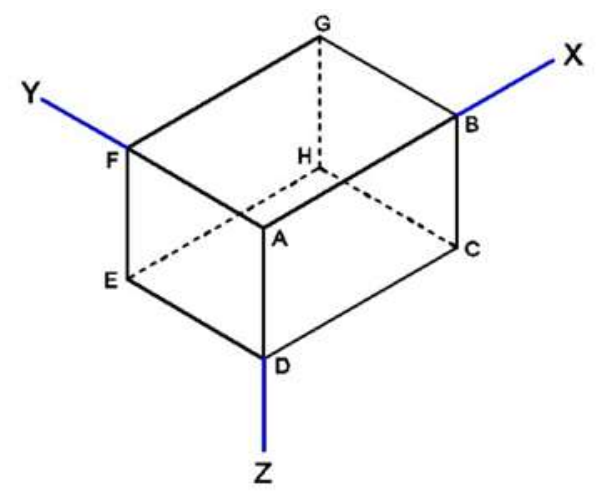

Fonte: Souza (2009)

Dessa forma, tem-se as seguintes condições de contorno:

a) Fronteira $A B C D$

Por se tratar de uma fronteira do domínio onde suas células vizinhas no sentido negativo do eixo $Y$ pertencem a um dos quadrantes do volume de solo total, dada a simetria, estabelece-se, então, uma condição de fluxo nulo.

$$
\mathrm{q}_{\mathrm{y}-}=\mathrm{K}_{\mathrm{MED}} \cdot \frac{\Delta \mathrm{H}_{\mathrm{y}}}{\Delta \mathrm{y}}=0 ; \mathrm{t} \geq 0 ; 0 \leq i \leq \mathrm{M}-1 ; j=0 ; 0 \leq \mathrm{k} \leq \mathrm{P}-1
$$

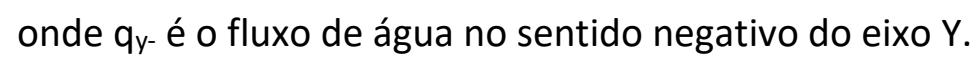

b) Fronteira ADEF

Condição semelhante à anterior, onde as células vizinhas no sentido negativo do eixo $X$ pertencem a um dos quadrantes do volume de solo total, estabelecendo-se, também, uma condição de fluxo nulo.

$$
\mathrm{q}_{\mathrm{X}-}=\mathrm{K}_{\mathrm{MED}} \cdot \frac{\Delta \mathrm{H}_{\mathrm{x}}}{\Delta \mathrm{x}}=0 ; \quad \mathrm{t} \geq 0 ; \mathrm{i}=0 ; 0 \leq \mathrm{j} \leq \mathrm{N}-1 ; 0 \leq \mathrm{k} \leq \mathrm{P}-1
$$

onde $q_{x}$ - é o fluxo de água no sentido negativo do eixo $X$.

c) Fronteira EFGH 
Considerando que as dimensões do domínio são definidas de tal forma que a frente de umedecimento não atinja esta fronteira, tem-se aqui, também, uma condição de fluxo nulo.

$$
\theta(i, j, k)=\theta_{0} ; \quad t \geq 0 ; 0 \leq i \leq M-1 ; j=N-1 ; 0 \leq k \leq P-1
$$

ou, de outra forma,

$$
\mathrm{q}_{\mathrm{y}+}=\mathrm{K}_{M E D_{\mathrm{y}}} \cdot \frac{\Delta \mathrm{H}_{\mathrm{y}}}{\Delta \mathrm{y}}=0 ; \quad \mathrm{t} \geq 0 ; 0 \leq \mathrm{i} \leq \mathrm{M}-1 ; \mathrm{j}=\mathrm{N}-1 ; 0 \leq \mathrm{k} \leq \mathrm{P}-1
$$

onde $\mathrm{q}_{\mathrm{y}^{+}}$é o fluxo de água no sentido positivo do eixo $\mathrm{Y}$.

d) Fronteira BCHG

Condição semelhante à anterior, resultando, novamente, em uma condição de fluxo nulo.

$$
\theta(i, j, k)=\theta_{0} ; \quad t \geq 0 ; i=M-1 ; 0 \leq j \leq N-1 ; 0 \leq k \leq P-1
$$

ou, de outra forma,

$$
q_{x+}=K_{M E D_{x}} \cdot \frac{\Delta H_{x}}{\Delta x}=0 ; \quad t \geq 0 ; i=M-1 ; 0 \leq j \leq N-1 ; 0 \leq k \leq P-1
$$

onde qx+ é o fluxo de água no sentido positivo do eixo $\mathrm{X}$.

e) Fronteira CDEH

Condição semelhante à anterior, resultando novamente em uma condição de fluxo nulo.

$\theta(i, j, k)=\theta_{0} ; \quad t \geq 0 ; 0 \leq i \leq M-1 ; 0 \leq j \leq N-1 ; k=P-1$

ou, de outra forma,

$$
\mathrm{q}_{\mathrm{z}+}=\mathrm{K}_{\mathrm{MED}} \cdot \frac{\Delta \mathrm{H}_{\mathrm{z}}}{\Delta \mathrm{z}}=0 ; \quad \mathrm{t} \geq 0 ; 0 \leq \mathrm{i} \leq \mathrm{M}-1 ; 0 \leq \mathrm{j} \leq \mathrm{N}-1 ; \mathrm{k}=\mathrm{P}-1
$$

onde $q_{z+}$ é o fluxo de água no sentido positivo do eixo $Z$. 


\section{f) Fronteira AFGB}

Nesta fronteira, tem-se duas situações que ocorrem em momentos distintos: a infiltração de água e a redistribuição.

Ao término da fase de infiltração, ou seja, durante a redistribuição, considerando não haver acúmulo de água na superfície do solo e desprezando o efeito evaporativo, tem-se, então, uma condição de fluxo nulo.

$$
\mathrm{q}_{\mathrm{Z}^{+}}=\mathrm{K}_{\mathrm{MED}} \cdot \frac{\Delta \mathrm{H}_{\mathrm{z}}}{\Delta \mathrm{z}}=0 ; \quad \mathrm{t}>0 ; 0 \leq \mathrm{i} \leq \mathrm{M}-1 ; 0 \leq \mathrm{j} \leq \mathrm{N}-1 ; \mathrm{k}=0
$$

Durante a infiltração, tem-se que, inicialmente, toda a água fornecida pelo gotejador adentra no solo através da célula $i=0, j=0, k=0$. Com o passar do tempo, esta célula alcança a condição de saturação, podendo-se concluir que sobre ela se forma uma zona de empoçamento, já que a taxa de infiltração se torna inferior à vazão do emissor. Dessa forma, também as células vizinhas tenderão à condição de saturação, aumentando, consequentemente e continuamente, a área do disco saturado, porção da fronteira por onde se infiltra grande parte da água do emissor. Este processo continua até que o raio do disco saturado se estabilize.

A infiltração de água nessa fronteira acontece pelo disco saturado, formando-se em sua volta uma região denominada de área superficial molhada, conforme apresentado por Souza et al. (2006).

Portanto, durante a infiltração e fora da área superficial molhada, tem-se uma condição semelhante à que se dá na fase de redistribuição, apresentada anteriormente pela equação (13).

Na zona saturada, tem-se a seguinte condição:

$$
\theta(i, j, k)=\theta_{s} ; \quad t>0 ; 0 \leq i \leq R_{\text {sat }} ; 0 \leq j \leq R_{\text {sat }} ; k=0
$$

onde $\theta_{\mathrm{s}}$ é a umidade volumétrica do solo no estado de saturação, e $R_{\text {sat }}$ é o raio do disco saturado no intervalo de tempo considerado, tendo em vista que a área saturada é crescente até o momento da sua estabilização.

Da mesma maneira, o tempo também necessita ser discretizado em intervalos convenientes de forma a se adequar à discretização da equação de escoamento. Para permitir um controle mais adequado do 
processo durante a utilização do modelo, opta-se por duas formas de escolha do incremento de tempo. Na primeira, o incremento de tempo é fixo e definido no início da simulação. Na segunda forma, o tempo é variável e se escolhe apenas o primeiro intervalo. Os incrementos de tempo seguintes são determinados usando uma equação proposta por Zaradny (1978):

$$
t^{i} \leq \frac{F A C \cdot \Delta x}{q\left(t^{i-1}\right)}
$$

onde $\Delta \mathrm{t}^{\mathrm{i}}$ é o intervalo de tempo calculado, $\Delta \mathrm{x}$ é o tamanho da célula numa determinada direção (ou a distância entre nós adjacentes) e q é o fluxo ocorrido na célula no instante de tempo anterior. 0 coeficiente FAC corresponde à máxima variação do teor de umidade permitida entre qualquer célula da malha. Pode variar de 0,015 a 0,035 $\mathrm{cm}^{3} \mathrm{~cm}^{-3}$, sendo o menor valor usado para os períodos de movimento rápido (infiltração e início da redistribuição) e o maior valor usado para períodos de movimento lento, como por exemplo, durante longos períodos de redistribuição.

Considerando, então, a discretização do domínio, as vazões entre uma célula genérica i, j, k e sua vizinha na direção positiva do eixo $\mathrm{X}$, são dadas por:

$$
Q_{i+1, j, k}^{i, j, k}=K_{i+1, j, k}^{i, j, k} \cdot \frac{H_{i, j, k}-H_{i+1, j, k}}{\Delta x} \cdot \Delta y \cdot \Delta z
$$

onde os sobrescritos e subscritos colocados após a vazão do gotejador Q e da condutividade hidráulica $\mathrm{K}$, representam, respectivamente, a posição da célula considerada e da sua vizinha.

Opta-se por usar duas formas de cálculo para a condutividade hidráulica média entre células vizinhas: média aritmética e média ponderada, adotando-se como peso para a ponderação, as umidades volumétricas das células. Vale salientar a importância deste valor médio, principalmente na vizinhança da frente de molhamento, onde a variação da condutividade hidráulica entre células vizinhas resulta em um valor muito elevado.

Considerando a condutividade hidráulica entre uma célula genérica i, j, k e suas vizinhas, tem-se para a média aritmética:

$$
K_{i+1, j, k}^{i, j, k}=\frac{K_{i, j, k}+K_{i+1, j, k}}{2}
$$


Para a média ponderada, tem-se:

$$
K_{i+1, j, k}^{i, j, k}=\frac{K_{i, j, k} \cdot \theta_{i, j, k}+K_{i+1, j, k} \cdot \theta_{i+1, j, k}}{\theta_{i, j, k}+\theta_{i+1, j, k}}
$$

A vazão resultante $(\Delta Q)$ em cada célula é obtida pelo somatório das vazões que entram e que saem, representando o resultado das interações entre uma célula genérica i, j, k e suas células vizinhas.

$$
\Delta Q_{i, j, k}=Q_{i+1, j, k}^{i, j, k}+Q_{i-1, j, k}^{i, j, k}+Q_{i, j+1, k}^{i, j, k}+Q_{i, j-1, k}^{i, j, k}+Q_{i, j, k+1}^{i, j, k}+Q_{i, j, k-1}^{i, j, k}
$$

O valor da vazão resultante pode ser positivo ou negativo, conforme a célula em questão esteja ganhando ou perdendo água, respectivamente. Pode ainda ser nulo, caso em que a célula está em equilíbrio dinâmico com suas vizinhas, ou quando não há fluxo.

A variação de umidade é obtida por:

$$
\Delta \theta_{\mathrm{i}, \mathrm{j}, \mathrm{k}}=\frac{\Delta \mathrm{Q}_{\mathrm{i}, \mathrm{j}, \mathrm{k}}}{\mathrm{V}_{\mathrm{i}, \mathrm{j}, \mathrm{k}}} \cdot \Delta \mathrm{t}
$$

A umidade final da célula é calculada por:

$$
\theta_{i, j, k}^{t+\Delta t}=\theta_{i, j, k}^{t}+\Delta \theta_{i, j, k}
$$

onde os índices t e $\mathrm{t}+\Delta \mathrm{t}$ representam, respectivamente, o tempo atual e o tempo seguinte e $\mathrm{V}_{\mathrm{i}, \mathrm{j}, \mathrm{k}}$ é o volume de cada célula.

Para a situação em que os bulbos molhados podem vir a se encontrar, é usada uma forma de cálculo semelhante à do bulbo isolado, fazendo-se as devidas adaptações nas condições de contorno da fronteira onde acontece a sobreposição.

Neste caso, considera-se que a linha lateral se encontra na direção do eixo $\mathrm{X}$ e que os gotejadores estão igualmente espaçados, conforme mostrado na Figura 3. 
FIGURA 3 - Vista em planta da linha lateral de gotejadores distribuídos ao longo do eixo X

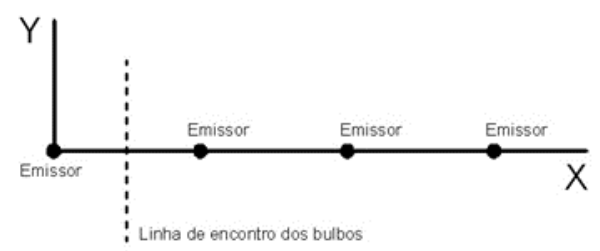

Fonte: Souza (2009)

A linha de encontro dos bulbos coincide com a fronteira BCHG, que tem sua nova condição de contorno dada simplesmente por:

$$
\mathrm{q}_{\mathrm{x}+}=\mathrm{K}_{\mathrm{MED}} \cdot \frac{\Delta \mathrm{H}_{\mathrm{x}}}{\Delta \mathrm{x}}=0 ; \quad \mathrm{t}>0 ; \mathrm{i}=\mathrm{M}-1 ; 0 \leq \mathrm{j} \leq \mathrm{N}-1 ; 0 \leq \mathrm{k} \leq \mathrm{P}-1
$$

Todas as demais condições de fronteira se mantem inalteradas.

Conhecido o espaçamento entre os gotejadores, a linha de encontro dos bulbos está a meio caminho entre eles. Dessa forma, quando a frente de molhamento atinge esta linha de encontro, tem-se um movimento de água preponderantemente nas direções $Y$ e $Z$, dada às condições de simetria do perfil de solo em torno dos emissores, formando então uma faixa úmida ao longo da linha lateral.

Na Figura 4 é apresentada a sobreposição superficial de dois bulbos, onde S representa a distância de sobreposição, $S_{e}$ é o espaçamento entre emissores, e $r_{s}$ é o raio superficial do bulbo.

FIGURA 4 - Sobreposição superficial entre bulbos molhados

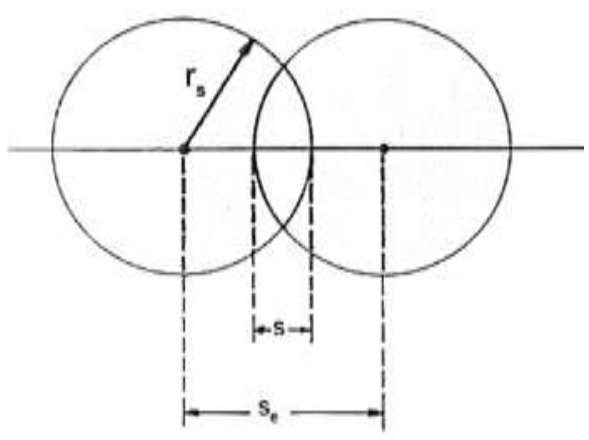

Fonte: Pizarro Cabello (1990)

A sobreposição, aqui chamada de $S_{p}$, é dada, então, por: 


$$
S_{p}=\frac{S}{r_{s}} \cdot 100
$$

e, sendo a distância de sobreposição (S) dada por:

$$
\mathrm{S}=2 \cdot \mathrm{r}_{\mathrm{s}}-\mathrm{S}_{\mathrm{e}}
$$

Baseado na formulação matemática, proposta e usada no modelo desenvolvido, foi elaborado um programa computacional denominado PSIGS (Programa de Simulação da Irrigação por Gotejamento Superficial), para simular o processo de movimento de água no solo após sua aplicação pelo método de irrigação por gotejamento superficial. A simulação se inicia com a aplicação de água por determinado período de tempo, podendo se prolongar pelo tempo de redistribuição desejado. Os resultados obtidos nas simulações do programa computacional desenvolvido foram validados a partir de comparações com dados de campo e resultados de simulações de outros modelos. Estas comparações demonstraram que o modelo apresenta resultados confiáveis e pode ser utilizado como ferramenta para o dimensionamento de instalações de irrigação por gotejamento (SOUZA, 2009).

Realizou-se um balanço de volume com o propósito de validar o modelo em termos do princípio de conservação de massa, comparando-se o volume de água armazenado no solo antes e após a aplicação de água pelo gotejador. Vale salientar que o efeito evaporativo não foi aqui considerado. Para avaliar o modelo em termos da conservação de massa, determinou-se os volumes iniciais e finais obtidos em cada simulação, bem como os volumes de água aplicados pelos gotejadores e os respectivos indicadores de conservação de massa, $\mathrm{O}$ erro de conservação de massa determinado pelo programa computacional PSIGS, ao simular o modelo desenvolvido, foi praticamente desprezível, seja para bulbo molhado isolado ou com sobreposição. Este comportamento é característico do método dos volumes finitos aplicado ao modelo, por garantir a determinação da diferença dos fluxos de água entre um volume de controle e os seus volumes adjacentes. A resolução da equação de escoamento de forma não acoplada, ou seja, independente da resolução da equação em outra célula, tornou os resultados finais bastante confiáveis, não apresentando perdas ou ganhos excessivos de massa durante o processamento computacional (SOUZA, 2009).

E, como forma de avaliar o comportamento do modelo em relação à variação dos parâmetros de entrada, foi feita uma análise de sensibilidade do mesmo. Os parâmetros de entrada utilizados, para esta análise, foram umidade inicial, condutividade hidráulica do solo saturado, vazão do gotejador, 
incremento do tempo usado na simulação e dimensões das células (volumes de controle integrantes do domínio). Estes parâmetros foram, então, incrementados e decrementados em relação ao seu valor padrão, mantendo-se todos os outros dados de entrada fixos, observando-se a sensibilidade do modelo a estas alterações. A análise de sensibilidade realizada a partir da variação de alguns parâmetros do solo e do emissor, demonstrou que, com relação à umidade final dentro do bulbo, o modelo é relativamente sensível tanto a variações positivas quanto negativas desses parâmetros (SOUZA, 2009).

\section{RESULTADOS E DISCUSSÃO}

Avaliou-se a formação dos bulbos molhados isolados na fase de infiltração, obtidos pelo modelo para dois intervalos de tempo, usando-se três solos com diferentes texturas, comparando-se a forma e as dimensões dos bulbos. Para a simulação, considerou-se as seguintes condições para os três solos:

umidade inicial: $0,25 \mathrm{~cm}^{3} \mathrm{~cm}^{-3}$;

vazão do gotejador: 3,0 L h-1;

tempo de aplicação de água: 120 min; e

tempo de simulação: 120 min.

As características físico-hídricas dos solos utilizados nas simulações e os seus respectivos parâmetros de ajuste, cujas informações foram obtidas de Miranda e Duarte (2002), são apresentadas nas Tabelas 1 e 2. De acordo com a classificação textural, os solos 1, 2 e 3, são respectivamente classificados em franco arenoso (sandy loam), argilo-arenoso (sandy clay) e argiloso (clay).

TABELA 1 - Características físico-hídricas dos solos usados na simulação

\begin{tabular}{|c|c|c|c|c|c|c|c|}
\hline \multirow[b]{2}{*}{$\begin{array}{c}\text { Tipo de } \\
\text { solo }\end{array}$} & \multicolumn{3}{|c|}{ Textura } & \multicolumn{2}{|c|}{ Densidade } & \multirow[b]{2}{*}{$\begin{array}{c}\text { Porosidade } \\
\%\end{array}$} & \multirow[b]{2}{*}{$\begin{array}{c}\mathrm{K}_{\mathrm{s}} \\
\mathrm{cm} \mathrm{h}^{-1}\end{array}$} \\
\hline & Areia & $\begin{array}{c}\text { Silte } \\
\%\end{array}$ & Argila & ${ }_{\mathrm{K}}$ Solo & $\begin{array}{l}\text { Partículas } \\
\mathrm{Im}^{-3}\end{array}$ & & \\
\hline Solo 1 & 69,5 & 12,0 & 18,5 & 1,560 & 2,575 & 39,41 & 18,19 \\
\hline Solo 2 & 55,0 & 7,0 & 38 & 1,2 & & & 16,18 \\
\hline Solo 3 & 12,0 & 25,0 & 63,0 & 1,330 & 2,580 & 48,44 & 8,73 \\
\hline
\end{tabular}

TABELA 2 - Parâmetros de ajuste usando o modelo de van Genuchten (1980)

\begin{tabular}{|c|c|c|c|c|}
\hline \multirow[b]{2}{*}{$\begin{array}{l}\text { Tipo de } \\
\text { solo }\end{array}$} & \multicolumn{4}{|c|}{ Parâmetros hidrodinâmicos do solo } \\
\hline & $\overline{\theta_{r}}$ & $\theta_{\mathrm{s}}$ & $\begin{array}{c}\alpha \\
\mathbf{c m}^{-1}\end{array}$ & $\mathbf{n}$ \\
\hline Solo 1 & 0,162 & 0,443 & 0,0449 & 3,6732 \\
\hline Solo 2 & 0,164 & 0,562 & 0,1265 & 3,9898 \\
\hline Solo 3 & 0,207 & 0,645 & 0,0429 & 1,4250 \\
\hline
\end{tabular}


Nas Figuras 5, 6 e 7 são apresentadas as isolinhas de umidade do bulbo molhado para os tempos de 60 e 120 min de simulação, usando os solos 1, 2 e 3, respectivamente.

A forma e as dimensões dos bulbos molhados formados se apresentaram de acordo com o esperado. No solo 1, o mais arenoso de todos, o bulbo molhado teve o maior alcance vertical, $\mathrm{z}=41,3 \mathrm{~cm} \mathrm{e} \mathrm{z}=$ $59,9 \mathrm{~cm}$ para os tempos de $60 \mathrm{~min}$ e $120 \mathrm{~min}$, respectivamente. Estes valores foram maiores que duas vezes as profundidades alcançadas pelo solo 3 , o mais argiloso, que teve $z=20,4 \mathrm{~cm}$ e $z=25,7 \mathrm{~cm}$, para os mesmos tempos considerados.

FIGURA 5 - Isolinhas de umidade obtidas pelo modelo para os tempos de simulação de 60 e 120 min, usando um solo franco arenoso (sandy loam)

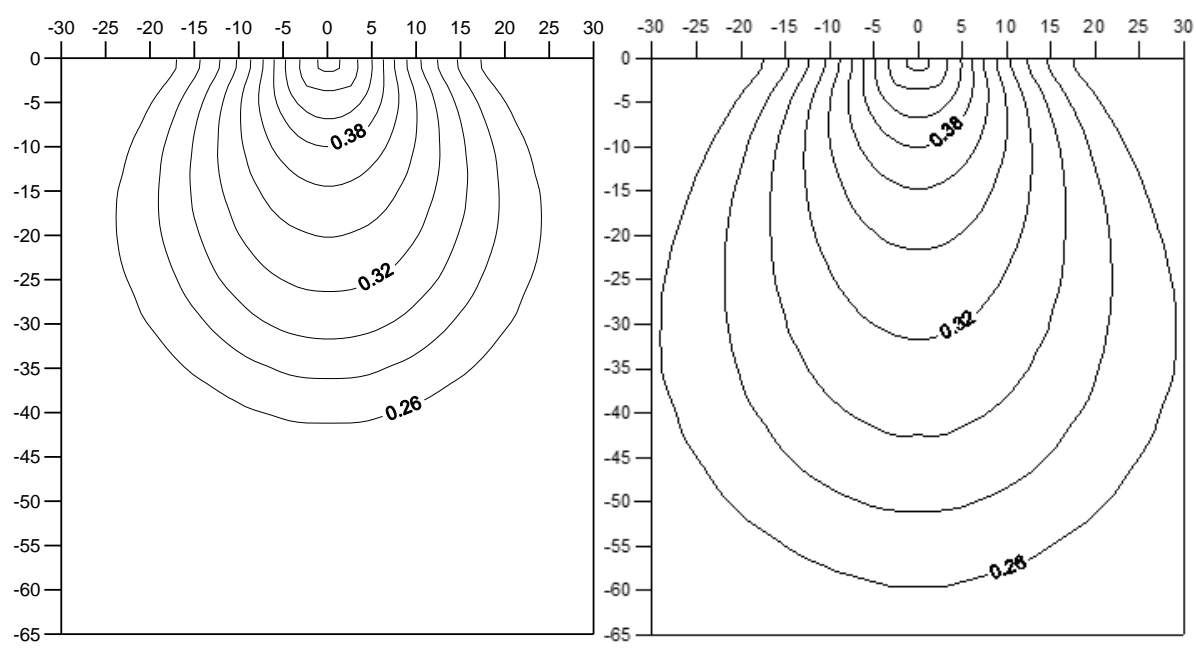

FIGURA 6 - Isolinhas de umidade obtidas pelo modelo para os tempos de simulação de 60 e 120 min, usando um solo argilo-arenoso (sandy clay)

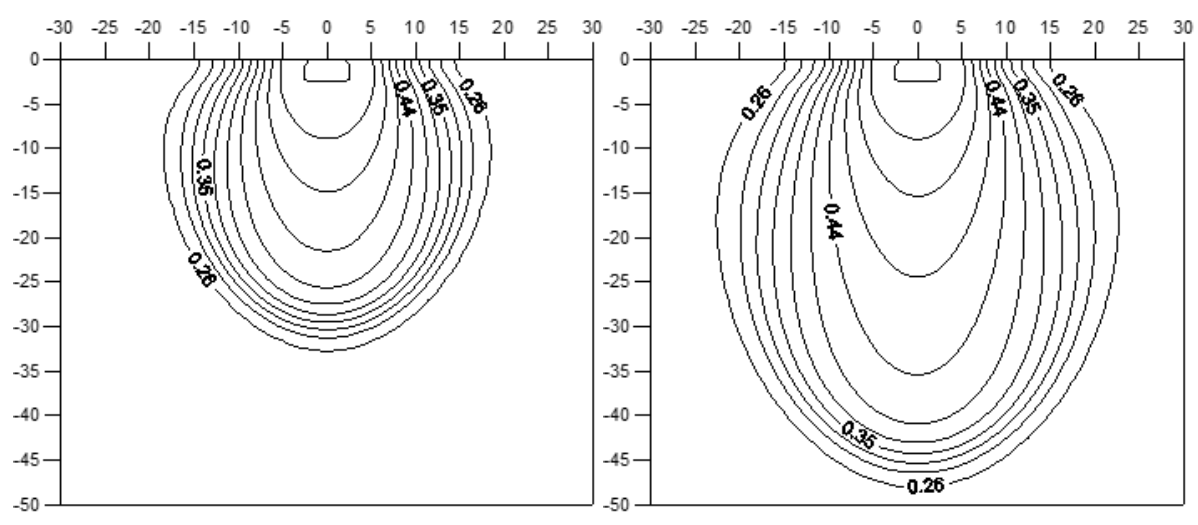


FIGURA 7 - Isolinhas de umidade obtidas pelo modelo para os tempos de simulação de 60 e 120 min, usando um solo argiloso (clay)

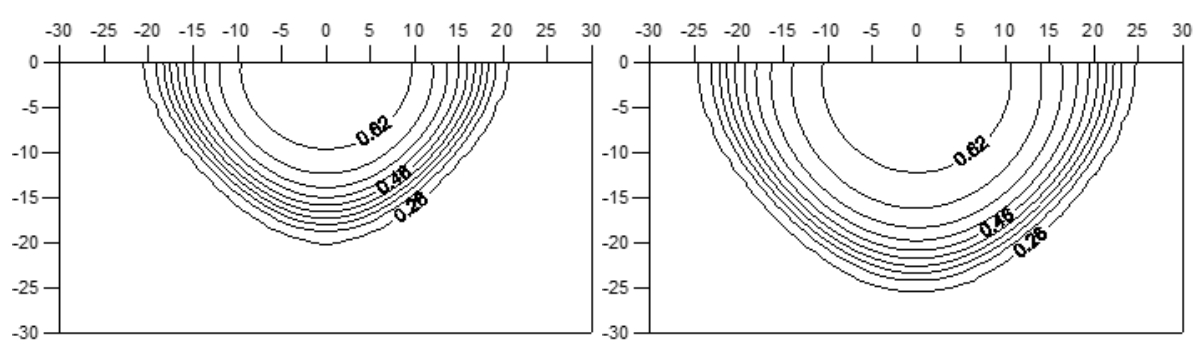

Com relação aos raios dos bulbos na superfície do solo, pode-se perceber que para os solos 1 e 2 , seus valores praticamente não diferiram para os tempos de $60 \mathrm{~min}$ e $120 \mathrm{~min}$, indicando que seus raios superficiais se estabilizaram antes de $60 \mathrm{~min}$. No caso do solo 3, o raio do bulbo na superfície continua a crescer após o tempo de $60 \mathrm{~min}$. Este comportamento se deve certamente ao elevado teor de argila do solo, corroborando também a condutividade hidráulica do solo saturado, cujo valor é o menor entre os três solos. Os valores dos raios e das profundidades do bulbo molhado para todos os solos e todos os tempos são mostrados na Tabela 3.

TABELA 3 - Valores do raio na superfície $\left(r_{s}\right)$, raio máximo $\left(r_{\text {máx }}\right)$ e profundidade $(z)$ alcançados pelo bulbo molhado para os tempos de simulação de 60 e $120 \mathrm{~min}$, obtidos pelo modelo para solos de diferentes texturas

\begin{tabular}{|c|c|c|c|c|c|c|c|c|c|}
\hline \multirow{2}{*}{$\begin{array}{c}\text { Tempo } \\
\text { min }\end{array}$} & \multicolumn{3}{|c|}{ Franco arenoso } & \multicolumn{3}{|c|}{ Argilo-arenoso } & \multicolumn{3}{|c|}{ Argiloso } \\
\hline & $\mathbf{r}_{\mathbf{s}}$ & $\begin{array}{c}\mathbf{r}_{\text {máx }} \\
\mathbf{c m}\end{array}$ & $\mathbf{z}$ & $\mathbf{r}_{\mathbf{s}}$ & $\begin{array}{c}\mathbf{r}_{\text {máx }} \\
\mathbf{c m}\end{array}$ & $\mathbf{z}$ & $\mathbf{r}_{\mathbf{s}}$ & $\begin{array}{c}\mathbf{r}_{\text {máx }} \\
\mathbf{c m}\end{array}$ & $\mathbf{z}$ \\
\hline 60 & 17,0 & 24,2 & 41,3 & 14,4 & 18,6 & 32,9 & 20,8 & 21,0 & 20,4 \\
\hline 120 & 17,5 & 29,4 & 59,9 & 15,0 & 22,8 & 48,5 & 24,9 & 25,0 & 25,7 \\
\hline
\end{tabular}

De uma forma geral, os bulbos molhados avançaram mais na vertical para solos com maior capacidade de drenagem, e mais na horizontal para solos com maior capacidade de retenção.

Avaliou-se ainda a formação de faixa molhada pela sobreposição de bulbos molhados, obtidos pelo modelo para quatro intervalos de tempo, observando-se desde a formação do bulbo isolado até a sua sobreposição $\left(S_{p}\right)$ com o bulbo vizinho, a partir da variação do espaçamento entre os gotejadores $\left(S_{e}\right)$.

Os dados de entrada considerados para as simulações foram os seguintes:

umidade inicial do solo: $0,20 \mathrm{~cm}^{3} \mathrm{~cm}^{-3}$;

vazão do gotejador: $2,3 L^{-1}$; 
tempo de aplicação de água: 120 min;

tempo de simulação: 120 min; e

espaçamento entre gotejadores: 20,25 e $30 \mathrm{~cm}$.

O solo utilizado na simulação corresponde a um solo franco siltoso, cujas características texturais estão descritas em Fuentes, Haverkamp e Parlange (1992). A condutividade hidráulica do solo saturado (Ks) é de $12,625 \mathrm{~cm} \mathrm{~h}^{-1}$. Os parâmetros de ajuste da equação de van Genuchten (1980), para o solo são:

$\theta_{\mathrm{s}}=0,469 \mathrm{~cm}^{3} \mathrm{~cm}^{-3}$

$\theta_{\mathrm{r}}=0,1903 \mathrm{~cm}^{3} \mathrm{~cm}^{-3}$

$\alpha=0,00505 \mathrm{~cm}^{-1}$; e

$n=7,6336$.

Nas Figuras 8, 9, 10, 11, 12 e 13 são apresentados os bulbos molhados formados para os espaçamentos entre os gotejadores de 20, 25 e 30 cm, após decorridos os tempos de 30, 60, 90 e 120 min de aplicação de água.

FIGURA 8 - Vista superior das isolinhas de umidade da faixa molhada considerando espaçamento entre emissores de $20 \mathrm{~cm}$, para os tempos de 30,60, 90 e $120 \mathrm{~min}$, respectivamente

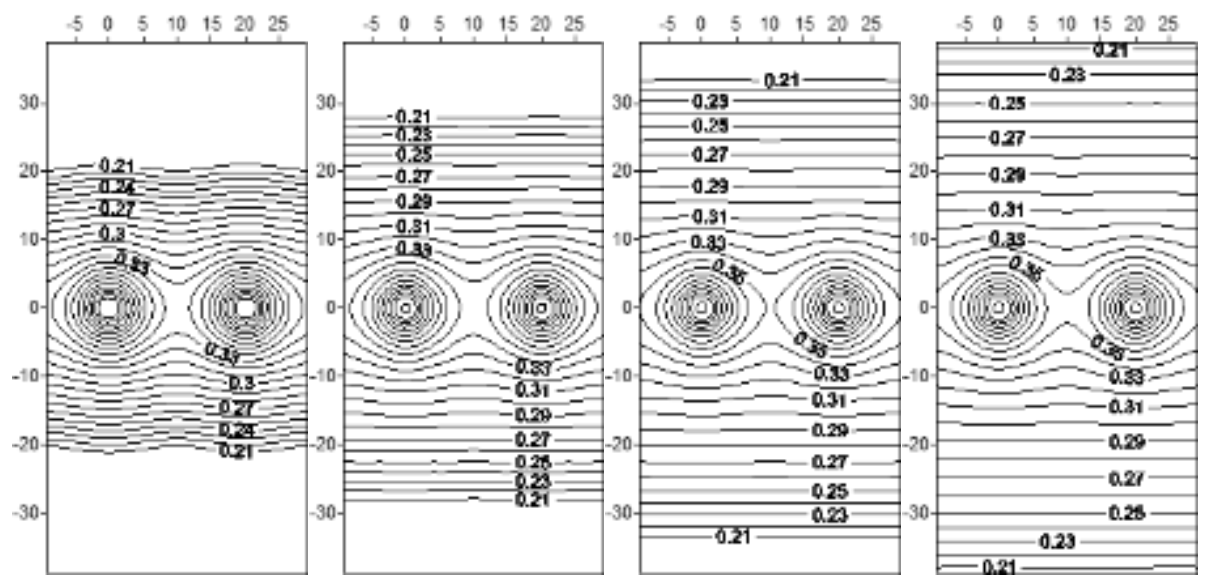


FIGURA 9 - Vista em corte vertical das isolinhas de umidade da faixa molhada considerando espaçamento entre emissores de $20 \mathrm{~cm}$, para os tempos de 30, 60, 90 e $120 \mathrm{~min}$, respectivamente

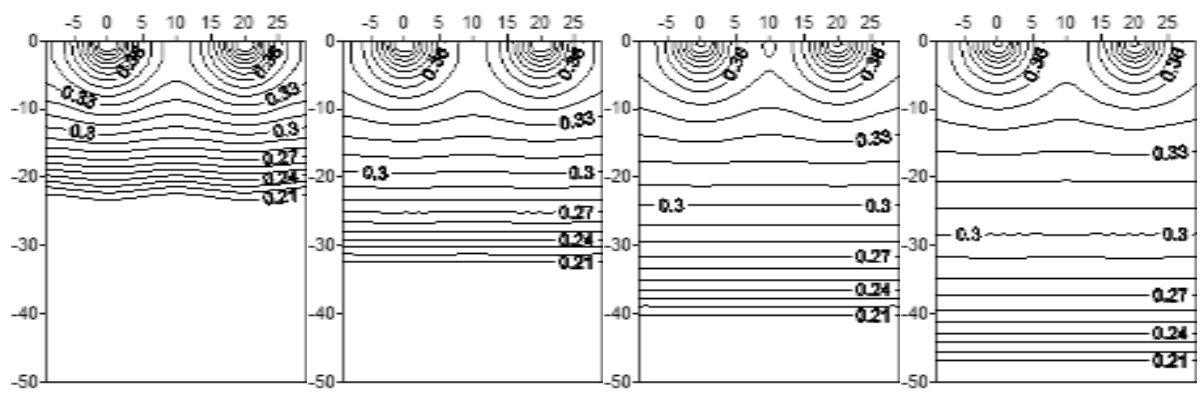

FIGURA 10 - Vista superior das isolinhas de umidade da faixa molhada considerando espaçamento entre emissores de $25 \mathrm{~cm}$, para os tempos de 30, 60, 90 e $120 \mathrm{~min}$, respectivamente

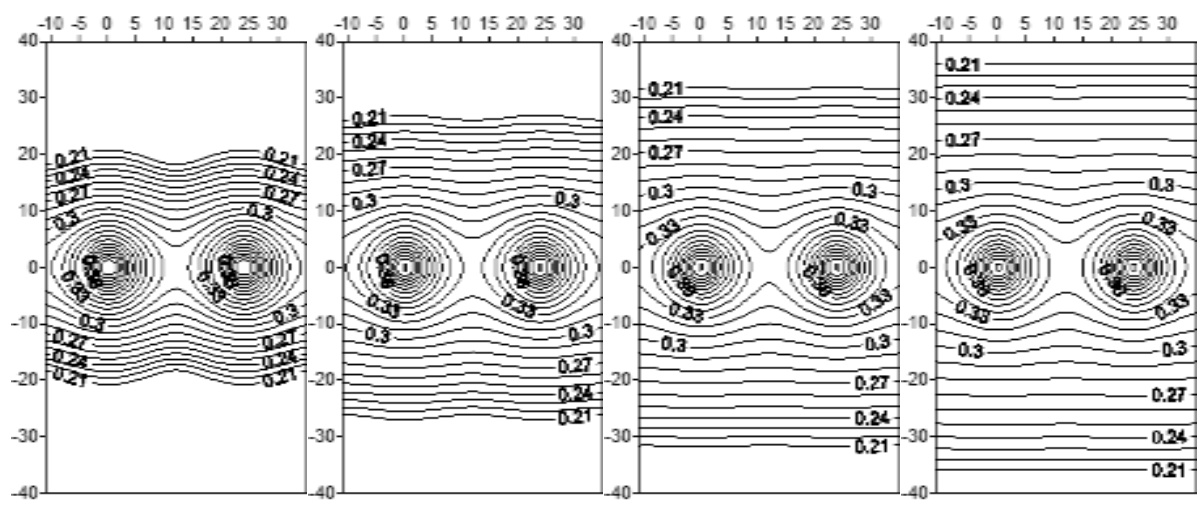

FIGURA 11 - Vista em corte vertical das isolinhas de umidade da faixa molhada considerando espaçamento entre emissores de $25 \mathrm{~cm}$, para os tempos de 30, 60, 90 e $120 \mathrm{~min}$, respectivamente

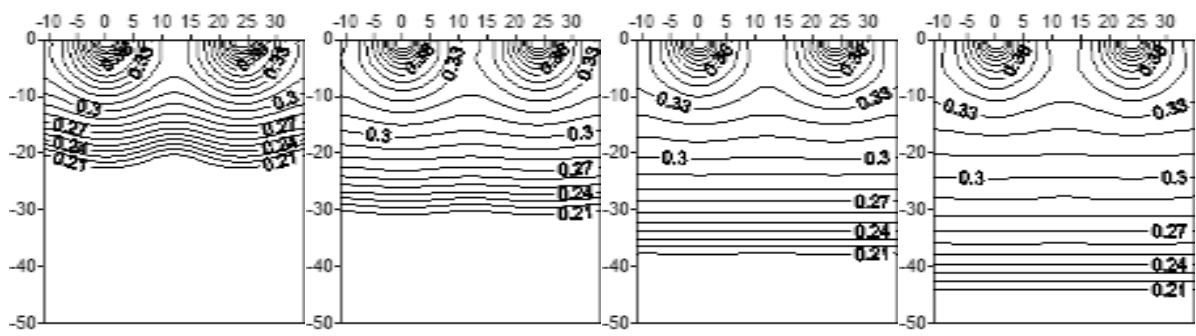


FIGURA 12 - Vista superior das isolinhas de umidade da faixa molhada considerando espaçamento entre emissores de $30 \mathrm{~cm}$, para os tempos de 30, 60, 90 e $120 \mathrm{~min}$, respectivamente

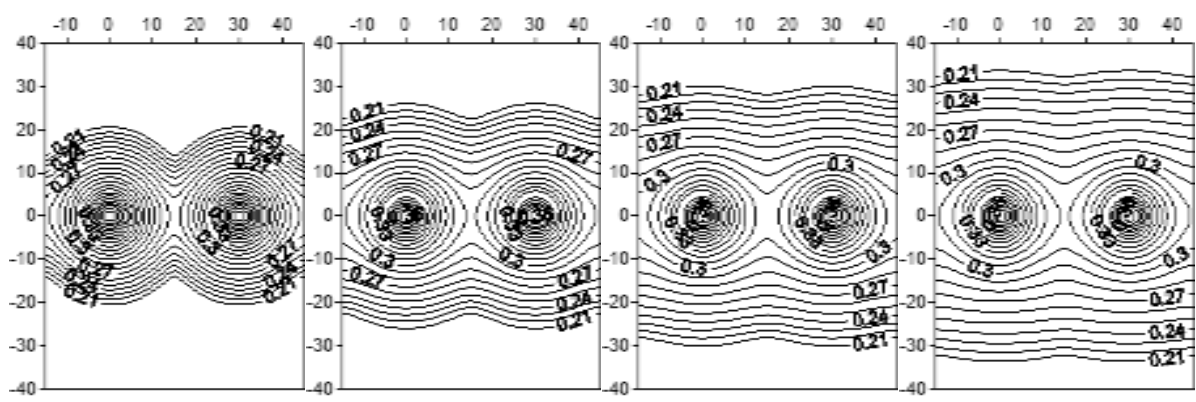

FIGURA 13 - Vista em corte vertical das isolinhas de umidade da faixa molhada considerando espaçamento entre emissores de $30 \mathrm{~cm}$, para os tempos de 30, 60, 90 e $120 \mathrm{~min}$, respectivamente

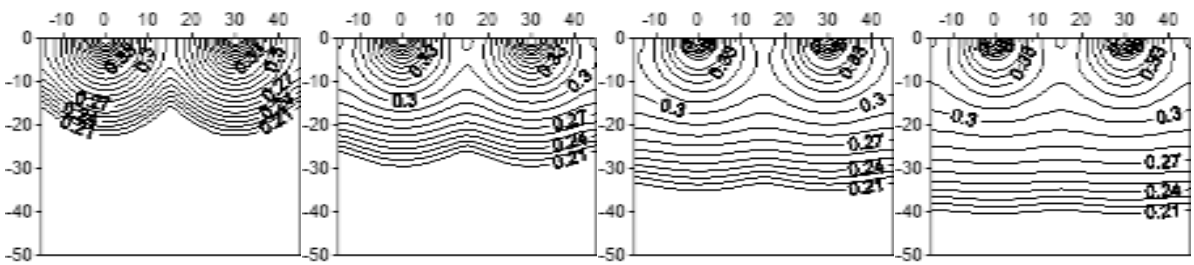

Para os tempos e espaçamentos entre emissores utilizados na simulação, a sobreposição entre bulbos molhados $\left(S_{p}\right)$ variou de $55,1 \%$ a $138,8 \%$. Para $S_{e}=30 \mathrm{~cm}$, obteve-se raio molhado na superfície do solo $\left(r_{s}\right)$ igual a $26 \mathrm{~cm}$ para o tempo de aplicação de água de $60 \mathrm{~min}$. De acordo com as equações (23) e (24), tem-se que a distância de sobreposição (S) foi de $22 \mathrm{~cm}$, conduzindo a um valor de $S_{p}=84,6 \%$. A profundidade alcançada (z), para este tempo e espaçamento, foi de $29,8 \mathrm{~cm}$. Os valores de sobreposição e profundidade alcançada para todos os tempos de aplicação de água e espaçamentos entre emissores são mostrados na Tabela 4.

TABELA 4 - Valores da sobreposição entre bulbos molhados $\left(S_{p}\right)$ e profundidade $(z)$ alcançada pelo bulbo, para os tempos de 30,60, 90 e $120 \mathrm{~min}$, com espaçamento entre emissores de 20, 25 e $30 \mathrm{~cm}$

\begin{tabular}{cccccccc}
\hline Tempo & $\mathbf{r}_{\mathbf{s}}$ & \multicolumn{2}{c}{ Esp. 20 cm } & \multicolumn{2}{c}{ Esp. 25 cm } & \multicolumn{2}{c}{ Esp. 30 cm } \\
min & $\mathbf{c m}$ & $\mathbf{S}_{\mathbf{p}}(\%)$ & $\mathbf{z}(\mathbf{c m})$ & $\mathbf{S}_{\mathbf{p}}(\%)$ & $\mathbf{z} \mathbf{( c m})$ & $\mathbf{S}_{\mathbf{p}}(\%)$ & $\mathbf{z}(\mathbf{c m})$ \\
\hline 30 & 20,7 & 103,4 & 23,5 & 79,2 & 23,0 & 55,1 & 23,0 \\
60 & 26,0 & 123,1 & 32,5 & 103,8 & 30,8 & 84,6 & 29,8 \\
90 & 29,7 & 132,7 & 40,2 & 115,8 & 38,0 & 99,0 & 35,5 \\
120 & 32,7 & 138,8 & 47,0 & 123,5 & 43,9 & 108,3 & 40,5 \\
\hline
\end{tabular}

Caso se deseje limitar o valor da sobreposição, pode-se alterar o espaçamento entre gotejadores ou modificar o tempo de aplicação de água, ou ainda, a vazão do gotejador. Nas situações simuladas, considerando-se o tempo de aplicação de água de $60 \mathrm{~min}$ e se fixando a sobreposição em 30\%, 
teríamos como resultado um espaçamento entre emissores em torno de $45 \mathrm{~cm}$. Para o tempo de aplicação de água de $120 \mathrm{~min}$, considerando condições idênticas à anterior, teríamos espaçamento em torno de $55 \mathrm{~cm}$.

Desta forma, demonstra-se a aplicabilidade do modelo como ferramenta a ser utilizada no dimensionamento do espaçamento entre gotejadores na linha lateral, durante a fase de desenvolvimento de projetos de irrigação. Fixando-se um valor para a vazão do gotejador, pode-se simular a formação do bulbo molhado para diversos espaçamentos, adotando-se aquele que melhor atenda o sistema radicular das plantas, evitando-se, por exemplo, uma faixa molhada muita larga ou perda de água por percolação profunda.

\section{CONSIDERAÇÕES FINAIS}

A concepção do modelo aplicando o método dos volumes finitos se mostra consistente no que diz respeito à simulação do movimento de água no solo, após sua aplicação na irrigação por gotejamento.

A forma e as dimensões do bulbo molhado, na fase de infiltração, obtidos pelo modelo são correlatos com o esperado.

A simulação de solos com diferentes texturas se mostra adequada à realidade e condizente com a teoria.

A simulação da formação de faixa molhada permite definir o espaçamento entre gotejadores que melhor atenda aos parâmetros de projeto utilizados. 


\section{REFERÊNCIAS}

BERGER, I.A.G. Modelação da rega gota-a-gota: transferências de água e de nitratos no solo. 1994. 156p. Dissertação (Mestrado em Engenharia da Rega e dos Recursos Agrícolas), Instituto Superior de Agronomia, Universidade Técnica de Lisboa, Lisboa, 1994.

BOTREL, T.A. Simulação da distribuição espacial da água em solo irrigado com gotejador. 1988. 61p. Tese (Doutorado em Agronomia), Escola Superior de Agricultura Luiz de Queiroz, Universidade de São Paulo, Piracicaba, 1988.

BRANDT, A.; BRESLER, E.; DINER, N.; BEN-ASHER, J.; HELLER, J.; GOLDBERG, D. Infiltration from a trickle source: I. Mathematical models. Soil Science Society of America Journal, Madison, v.35, p.675-682, 1971.

COELHO, E.F.; OR, D. Modelo de distribuição de água e de potencial matricial no solo sob gotejamento com extração de água por raízes. Pesquisa Agropecuária Brasileira, Brasília, v.34, n.2, p.225-234, 1999.

COOK, F.J.; THORBURN, P.J.; FITCH, P.; BRISTOW, K.L. WetUp: a software tool to display approximate wetting patterns from drippers. Irrigation Science, Heidelberg, v.22, n.3-4, p.129-134, 2003.

DASBERG, S.; BRESLER, E. Drip irrigation manual. Bet Dagan: International Irrigation Information Center, 1985. 95p.

FUENTES, C.; HAVERKAMP, R.; PARLANGE, J. Parameter constraints on closed-form soil water relationships. Journal of Hydrology, Amsterdam, v.134, p.117-142, 1992.

LOYOLA, J.M.T.; PREVEDELLO, C.L. Modelos analíticos para predição do processo da redistribuição da água no solo. Revista Brasileira de Ciência do Solo, Viçosa, v.27, n.5, p.783-787, 2003.

MALISKA, C.R. Transferência de calor e mecânica dos fluidos computacional. 2.ed. Rio de Janeiro: LTC, 2004. 453p.

MIRANDA, J.H.; DUARTE, S.N. Modelo para simulação da dinâmica de nitrato em colunas verticais de solo não saturado. Revista Brasileira de Engenharia Agrícola e Ambiental, Campina Grande, v.6, n.2, p.235-241, 2002.

PIZARRO CABELLO, F. Riegos localizados de alta frecuencia (RLAF): Goteo, microaspersión, exudación. 2ed. Madrid: Ediciones Mundi-Prensa, 1990. 471p.

RAATS, P.A.C. Steady infiltration from point sources, cavities and basins. Soil Science Society of America Journal, Madison, v.35, p.689-694, 1971.

RIVERA, R.N.C. Modelagem da dinâmica da água e do potássio na irrigação por gotejamento superficial. 2004. 89p. Tese (Doutorado em Agronomia), Escola Superior de Agricultura Luiz de Queiroz, Universidade de São Paulo, Piracicaba, 2004.

SOUZA, C.F.; SILVA, E.F.F.; FOLEGATTI, M.V.; OR, D. Irrigação por gotejamento: disco saturado e área superficial molhada versus distribuição e armazenamento da água no solo. Revista Brasileira de Ciências Agrárias, Recife, v.1, n.1, p.65-71, 2006. 
SOUZA, L.A.A. Fluxo tridimensional de água no solo: aplicação de volumes finitos na simulação da irrigação por gotejamento superficial. 2009. 128p. Dissertação (Mestrado em Irrigação e Drenagem), Universidade Federal Rural do Semi-Árido, Mossoró, 2009.

TABUADA, M.A.; BERGER, I.A.G. Modelação numérica da rega localizada. Recursos Hídricos, Lisboa, v.19, n.2-3, p.7-20, 1998.

VAN GENUCHTEN, M.T. A closed-form equation for predicting the hydraulic conductivity of unsaturated soils. Soil Science Society of America Journal, Madison, v.44, p.892-898, 1980.

WARRICK, A.W. Time-dependent linearized infiltration: I. Point source. Soil Science Society of America Journal, Madison, v.38, n.3, p.383-386, 1974.

WOODING, R.A. Steady infiltration from a shallow circular pond. Water Resources Research, Washington, v.4, p.1259-1273, 1968.

ZARADNY, H. Boundary conditions in modeling water flow in unsaturated soils. Soil Science, Heidelberg, v.125, n.2, p.75-82, 1978. 


\section{Capítulo 3}

do:

\section{ANÁLISE DAS PROPRIEDADES ELETRÔNICAS EM FIOS E PONTOS QUÂNTICOS SEMICONDUTORES DE SI/SIGE}


Resumo: Sistemas semicondutores têm atraído grande atenção nas últimas décadas, devido seu potencial de aplicações em dispositivos. Neste trabalho foi estudarado estruturas com potencial de pontos e fios quântico, sendo utilizado o modelo de evolução temporal chamado Split-operator, que tem o objetivo de solucionar numericamente a equação de Schrödinger dependente do tempo e a partir dessa solução obter os autoestados do elétron. Para essas heteroestruturas quânticas foram analisados dois tipos de configuração de alinhamento de banda: tipo-I, no qual os portadores de cargas estão confinados no mesmo material e tipo-ll no qual os portadores de cargas estão em materiais diferentes. O material estudado foi Si/SiGe, que dependendo da concentração de Ge usado na liga SiGe essa heteroestrutura passa por essa mudança de alinhamento de banda. Os níveis de energia de ligação e total do exciton foi calculado para o ponto e fio quântico variando alguns parâmetros tais como: raio, altura, interface gradual e campo magnético. Para ambas as heteroestruturas, foi observado que as energias são suscetíveis a variações quando é alterado algum desses parâmetros.

Palavras-chave: Semicondutores. Pontos e fios quânticos. Si/SiGe. Tipo-I. Tipo-II. 


\section{INTRODUÇÃO}

Uma das maiores vertentes das pesquisas científicas é o estudo dos semicondutores, e isso acontece devido sua importância na fabricação de diversos dispositivos eletrônicos, tais como: celulares, computadores, GPS, televisões, etc. Além disso, os semicondutores são os maiores responsáveis pela diminuição do tamanho da maioria dos aparelhos eletrônicos, como aconteceu nas televisões e nos computadores.

Entre os materiais semicondutores se destacam o silício, o germânio e o gálio. O silício se destaca por ser um dos materiais semicondutores mais abundantes no planeta, tendo aplicação em transistores para chips, células solares e em diversas variedades dos circuitos eletrônicos. Assim como o silício, o germânio é muito utilizado na fabricação de transistores e diodos, no entanto o germânio se destaca pelas suas propriedades ópticas tendo grande aplicação em sensores de luz. $O$ gálio por sua vez, vem recebendo bastante atenção devida sua grande aplicação, em especial, na área de telecomunicações.

Atualmente os estudos relacionados aos semicondutores estão focados nas estruturas de baixa dimensão como: poços, anéis, fios e pontos quânticos, onde diversas aplicações tecnológicas para tais estruturas foram encontradas, devido às suas propriedades físicas e aplicações em dispositivos optoeletrônicos. O estudo dessas estruturas é justificado por possuírem uma alta eficiência, baixo consumo de energia, e podem apresentar uma química facilmente manipulável. Atualmente grande parte dos dispositivos eletrônicos utilizam semicondutores heteroestruturados.

Neste trabalho estudaremos dois tipos de sistemas de dimensão reduzida: fios e pontos quânticos. 0 primeiro é caracterizado por confinar portadores de cargas, elétrons e buracos, em duas dimensões, deixando apenas uma dimensão livre. Esses sistemas têm sido usados com sucesso na aplicação de sistema opto-eletrônico e sensores biológicos, onde requer um forte confinamento. Já nos pontos quânticos os portadores estão confinados nas três direções, se assemelhando a um átomo, onde o potencial do núcleo é substituído pelo potencial de confinamento, resultando em uma forte quantização dos níveis de energia, daí o nome de átomo artificial, como os pontos quânticos também são conhecidos. 


\section{REFERENCIAL TEÓRICO}

\section{SEMICONDUTORES}

Os materiais podem ser classificados de acordo com o movimento das cargas elétricas em seu interior, sendo classificados em condutores, isolantes ou semicondutores. Os condutores permitem a fácil movimentação dessas cargas, em geral, os metais se apresentam como bons condutores. Os materiais isolantes, também chamados não-condutores, tendem a dificultar ou até mesmo não permitir a movimentação das cargas elétricas, sendo necessária uma certa quantidade de energia para que o movimento dessas cargas seja possível, são exemplos de isolantes os plásticos e as borrachas. Semicondutores são materiais sólidos que não são bons condutores e nem isolantes e podem se diferenciar, destes, devido suas propriedades físicas, como, sua resistividade elétrica ou a dificuldade com que uma corrente elétrica passa através do material quando este está sob a ação de um campo (BALKANSKI \& WALLIS, 2000).

\section{BANDAS DE ENERGIA}

As bandas de energia são decorrentes da aproximação dos orbitais moleculares, essa aproximação se dá quando são agregadas grandes quantidades de átomos, formando um sólido. Quanto dois ou mais orbitais atômicos são combinados resulta na formação de orbitais moleculares, cuja diferença de energia entre eles diminuem à medida que aumenta o número de átomos que compõem essa ligação. Como consequência temos uma considerável diminuição do espaçamento entre os níveis de energia, de tal maneira que no lugar de níveis discretos são formados um conjunto de níveis ou estados de energia virtualmente contínuo. Esses intervalos são chamados de bandas energia (OLIVEIRA, 2006).

A diferença entre os materiais condutores, isolantes e semicondutores, pode ser explicado através das estruturas de bandas. No isolante (Fig. 1a), a camada de valência está separada da banda de condução por um gap, na qual para um elétron saltar da banda de valência para a banda de condução é necessário realizar uma perturbação no meio que forneça uma energia superior a energia do gap $\left(E_{g}\right)$. Assim como o isolante, o semicondutor (Fig. 1b) possui gap, no entanto, este é inferior ao de um isolante da ordem de $3 \mathrm{eV}$. No condutor (Fig. 1c), por sua vez, as bandas valência e condução estão superpostas indicando que não possuem energia de gap e, portanto, não dificulta a passagem de corrente elétrica. 
FIGURA 1- Estrutura de banda para (a) um isolante, (b) um semicondutor e (c) um condutor.

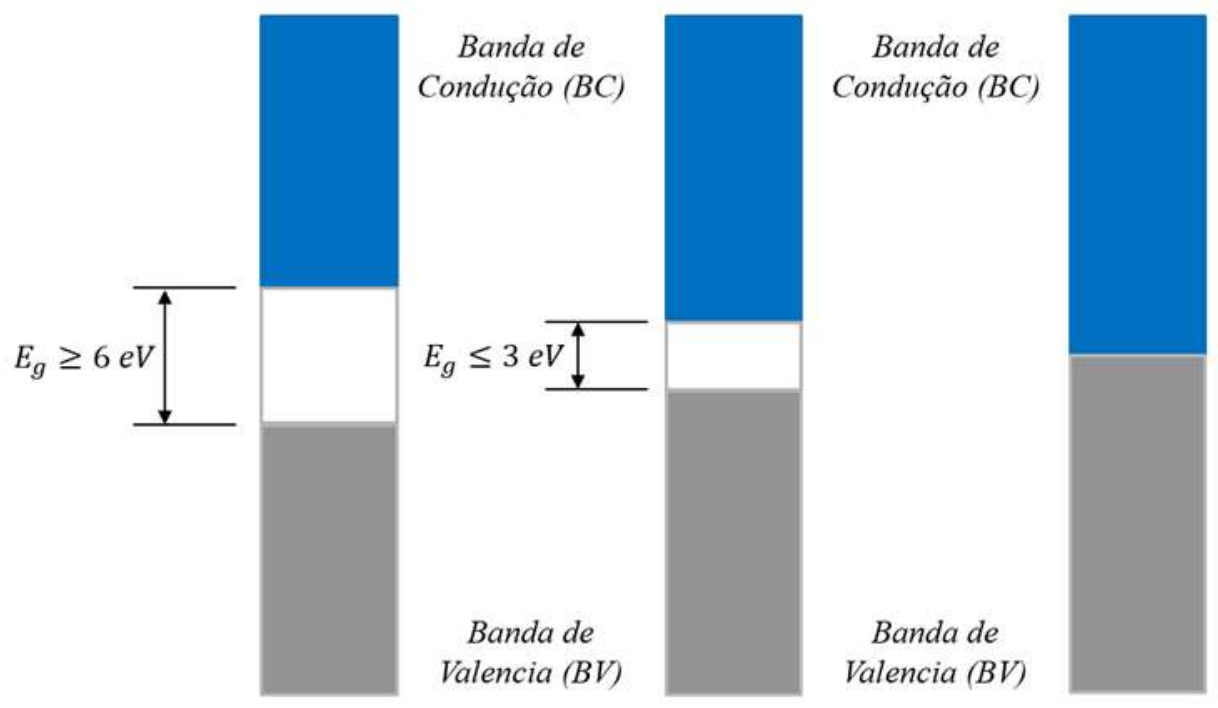

Fonte: Autoria própria.

Quando um elétron salta da banda de valência para a banda de condução, é deixado um estado desocupado que se comporta como uma partícula com carga positiva chamada de buraco (hole), a interação entre o elétron e o buraco é o que chamamos de exciton (CHAVES, 2007).

\section{HETEROESTRUTURAS}

Heteroestruturas são junções formadas por camadas justapostas de diferentes materiais semicondutores, que permite o confinamento dos portadores de carga (elétrons e buracos). Quando um semicondutor é formado por diferentes materiais provoca uma alteração na estrutura de banda dando origem ao que chamamos de poços e barreiras de potencial. Uma vez que os gaps de energia desses materiais são diferentes é gerada uma mudança abrupta semelhante a um degrau. As bandas de energias podem ser alinhados de duas maneiras: quando o alinhamento das bandas permite que $o$ exciton esteja confinado em apenas um material, esta estrutura é chamada tipo-I (Fig. 2a), quando o alinhamento é de tal forma que o elétron e o buraco estarão confinados em materiais diferentes chamamos de tipo-II (Fig. 2b) (CHAVES, 2007) (SILVA, 2008). 
FIGURA 2 - Representação esquemática do alinhamento de bandas tipo-I e tipo-II.

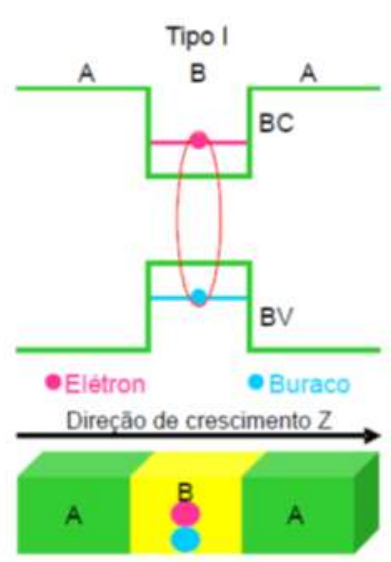

(a)

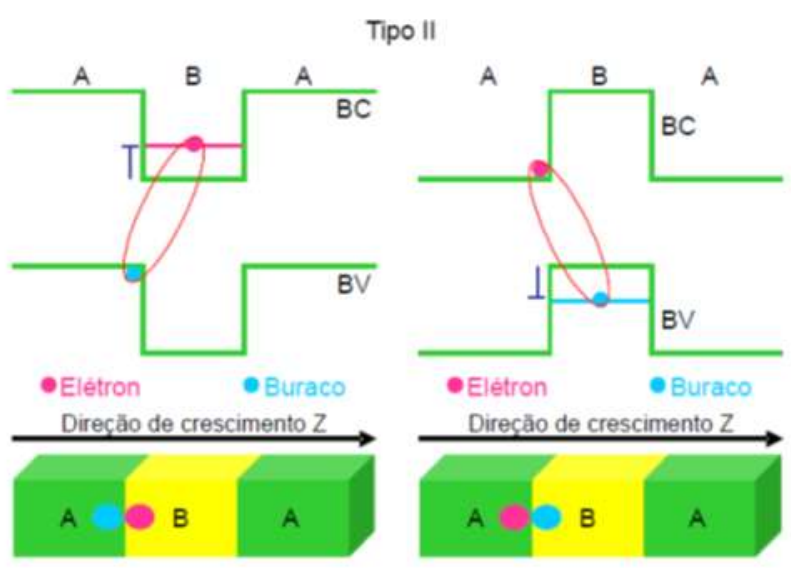

(b)

Fonte: Silva, 2008.

\section{EXCITON}

O exciton é formado quando um elétron e um buraco interagem entre si, a partir de uma ligação Coulombiana. Essa interação elétron-buraco movimenta-se por todo o cristal, no entanto esse par é eletricamente neutro. Em 1931, J. Frenkel definiu exciton como sendo a forte atração Coulombiana entre elétron e buraco localizados em cristais iônicos. Nos cristais iônicos os elétrons e buracos estão fortemente ligados devido às baixas constantes dielétricas, esse tipo de exciton é chamado de exciton de Frenkel. Outra definição de exciton foi dada por G. Wannier e N. F. Mott, o qual recebeu o nome de exciton de Wannier-Mott, esse tipo de exciton é caracterizado por ter uma ligação Coulombiana fraca, uma vez que a maioria destas ligações é enfraquecida devido os elétrons na camada de valência possuírem altas constantes dielétricas. As propriedades deste tipo de exciton podem ser determinadas usando o modelo de massa efetiva (SILVA, 2008).

A banda de condução dos semicondutores é muito instável, logo qualquer perturbação resultará com o elétron retornando para a banda de valência. Quando isso ocorre um fóton é emitido, essa energia liberada recebe o nome de energia do exciton. Em materiais na qual não existe o potencial de confinamento para os portadores a energia do exciton será:

$$
E_{\text {exc }}=E_{g}-\left|E_{b}\right|,
$$

na qual $E_{g}$ é a energia de gap e $E_{b}$ a energia de ligação devido à interação Coulombiana entre o elétron e o buraco. Para as heteroestruturas (poços, fios e pontos quânticos), deve-se levar em consideração à energia de confinamento. 


$$
E_{\text {exc }}=E_{g}+E_{e}+E_{h}-\left|E_{b}\right|,
$$

na qual $E_{e}$ e $E_{h}$ referem-se a energia dos portadores de carga, elétron e buraco respectivamente (SILVA, 2008).

\section{INTERFACES GRADUAIS}

Apesar das mais altas sofisticações nas técnicas de crescimentos ainda não é possível mudar abruptamente de material em uma heterojunção (SILVA, 2008). No entanto, na grande maioria dos estudos em semicondutores heteroestruturados é considerado a junção entre os materiais como sendo abrupto, o que torna os cálculos teóricos mais simples. Estudos experimentais comprovam a existência de uma região de interface entre os materiais, na qual, nessa região, existem átomos do material da barreira e do material do poço. Essa região na qual há transição dos dois materiais é o que chamamos de interfaces graduais. Essa região possui uma grande influência nas propriedades óticas das heteroestruturas semicondutoras.

Neste trabalho será utilizado o modelo proposto por Freire et al (1992), afim de tornar o trabalho mais realista. Pois neste modelo descreve a região interfacial, considerando que a massa e o potencial efetivo dos portadores são dependentes da fração molar dessa região:

$$
\begin{gathered}
m_{i}\left(z_{i}\right)=m^{*}\left[\mu_{i, 1} \chi\left(z_{i}\right)+\mu_{i, 2}\left(1-\chi\left(z_{i}\right)\right)\right] \\
V_{i}\left(z_{i}\right)=Q_{i}\left[\varepsilon_{1} \chi\left(z_{i}\right)+\varepsilon_{2} \chi^{2}\left(z_{i}\right)\right]
\end{gathered}
$$

com $i$ sendo os portadores de carga, elétron e buraco, $m^{*}$ a massa efetiva, $\mu_{i}$ os parâmetros experimentais da massa efetiva, $Q_{i}$ a band-offset, ou seja, as frações da diferença entre os gaps de energias dos diferente matérias que compõem o poço, $\chi(z)$ a função linear de $z$ na região intefacial que representa a fração molar, $\varepsilon_{1}$ e $\varepsilon_{2}$ constantes relacionadas a dependência composicional do gap (SILVA, 2008).

O modelo de massa efetiva tem se mostrado uma importante ferramenta para o estudo do movimento de elétrons e buracos em um sólido enquanto estão sujeitos a campos externos, impurezas, defeitos pontuais entre outros fatores. Este modelo traz uma aproximação para esse movimento baseando-se na ideia de transformar um problema inicialmente complexo em um problema mais simples, relacionando o movimento de um portador em um cristal sob a ação de um campo externo adicional ao movimento de um portador livre imerso em um mesmo potencial externo. Na qual todos os efeitos da estrutura cristalina estão considerados em um tensor denominado de massa efetiva, que possuem elementos determinados por cada tipo material que compõe sua estrutura (BITTENCOURT, 2002). 
De modo geral, a massa não é escalar, sendo considerada um tensor, apresentado como:

$$
m_{\alpha \beta}^{*}=\frac{\hbar^{2}}{\partial^{2} E / \partial k_{\alpha} \partial k_{\beta}}
$$

na qual $\alpha$ e $\beta$ são as direções da rede cristalina (REZENDE, 1996).

\section{FIO QUÂNTICO}

Fios quânticos são estruturas onde os portadores de cargas estão confinados em duas direções e livre em uma. Neste capítulo estudaremos fios quânticos de Si/SiGe. Calcularemos os níveis de energia dos portadores e energia do exciton. Para esses fios serão considerados dois tipos de alinhamentos de banda: tipo-I e tipo-II.

\section{MÉTODO SPLIT-OPERATOR}

O problema em questão trata de solucionar numericamente a equação de Schrödinger dependendo do tempo e a partir dessa solução obter os autoestados do elétron em um fio quântico. Para solucionar foi utilizado o método Split-operator que reduz os cálculos transformando um problema com $n$ variáveis em uma sequência de problemas unidimensionais (TAHA; ABLOWITZ, 1984).

Dada a equação de Schrödinger dependente do tempo:

$$
i \hbar \frac{\psi(r, t)}{d t}=H \psi(r, t)
$$

Uma solução formal para a equação 3.1 é:

$$
\psi(r, t+\Delta t)=e^{\frac{-i}{\hbar} H \Delta t} \psi(r, t)
$$

Na função exponencial da equação 6 pode ser considerado como um gerador de translação temporal, ou seja, um operado de evolução temporal. Portando, para resolvê-la é necessário conhecer o Hamiltoniano que descreve esse problema. Reescrever a equação 7 na forma de Cayley (HERCULANO, 2005):

$$
\begin{aligned}
& e^{\frac{-i}{\hbar} H \Delta t} \psi(r, t)=\frac{1+\frac{i}{2 \hbar} H \Delta t}{1-\frac{i}{2 \hbar} H \Delta t} \psi(r, t)=\psi(r, t+\Delta t), \\
& \left(1-\frac{i}{2 \hbar} H \Delta t\right) \psi(r, t+\Delta t)=\left(1+\frac{i}{2 \hbar} H \Delta t\right) \psi(r, t),
\end{aligned}
$$


resultando em um Hamiltoniano da seguinte forma:

$$
H=\frac{-\hbar^{2}}{2 m} \frac{d^{2}}{d x} V(r)
$$

na qual essas derivadas podem ser calculados usando o método de Crank-Nicolson, no qual consiste de reescrever estas derivadas na forma de diferencias finitas, discretizando o espaço e o potencial. Uma vez que a função de onda estará discretizada em pontos $i=1,2,3 \ldots$, podemos então formar matrizes coluna em ambos os lados da equação 9. Dessa forma, é necessário solucionar esse sistema de matrizes para determinar a função de onda em cada instante de tempo (HERCULANO, 2005).

No entanto, ao considerar o sistema como unidimensional obteremos matrizes tridiagonais, para o caso de um sistema bidimensional teremos matrizes pentagonais, para o sistema tridimensional, matrizes heptadiagonais, assim por diante. Quanto mais dimensões o problema tiver mais complexos são os cálculos para solução da equação de Schrödinger. Neste trabalho será analisado um sistema tridimensional, portanto será necessária a utilização do método Split-operator, que transforma esse problema em outros de matrizes tridiagonais (TAHA; ABLOWITZ, 1984).

Para isso o exponencial do Hamiltoniano será dividido em uma parte cinética e outro potencial:

$$
e^{\frac{-i}{\hbar} H \Delta t}=e^{\frac{-i}{2 \hbar} V \Delta t} e^{\frac{-i}{2 \hbar} T \Delta t} e^{\frac{-i}{2 \hbar} V \Delta t}
$$

Os operadores $T$ e $V$ não comutam, ou seja, não se pode igualar a exponencial da soma com o produto das exponenciais. Portanto, a equação 11 é a melhor aproximação para separar o exponencial do Hamiltoniano. O erro envolvido é da ordem de $\Delta t^{3}$, sendo desprezível, uma vez que os valores de $\Delta t$ já são muito pequenos.

Os termos que envolvem o potencial não envolvem derivadas podendo serem multiplicados ponto a ponto pela função de onda. Já o termo de energia cinética pode ser dividido em mais três partes, uma para cada direção, na forma de $\exp [T]=\exp [T x] \exp [T y] \exp [T z]$, uma vez que os três termos comutam entre si de forma exata. Assim, a exponencial não precisa ser aplicada nos três termos ao mesmo tempo e sim aplicado a cada direção um a um, tornando o problema computacionalmente mais fácil, pois troca a matriz heptadiagonal em três matrizes tridiagonais.

Aplicando a aproximação dada pela equação 11 a equação 7 obtemos:

$$
\psi(r, t+\Delta t)=e^{\frac{-i}{2 \hbar} V \Delta t} e^{\frac{-i}{2 \hbar} T \Delta t} e^{\frac{-i}{2 \hbar} V \Delta t} \psi(r, t) .
$$


Como função de onda $\psi(r, t+\Delta t)=\left|\psi_{i}\right\rangle_{t}$, o potencial e o espaço estão discretizados, basta aplicar a primeira exponencial ponto a ponto. Inicialmente chamaremos

$$
\xi_{i}=e^{\frac{-i}{2 \hbar} V_{i} \Delta t}\left|\psi_{i}\right\rangle_{t}
$$

e aplicando a exponencial com termo cinético função descrita na equação 13:

$$
\eta_{i}=e^{\frac{-i}{2 \hbar} T \Delta t} \xi_{i}
$$

Utilizando a forma de Cayley dada na equação 9:

$$
\begin{gathered}
\eta_{i}=\frac{1+\frac{i}{2 \hbar} T \Delta t}{1-\frac{i}{2 \hbar} T \Delta t} \xi_{i} \\
\left(1-\frac{i}{2 \hbar} T \Delta t\right) \eta_{i}=\left(1+\frac{i}{2 \hbar} T \Delta t\right) \xi_{i}
\end{gathered}
$$

Aplicando, nas derivadas do termo cinético, a discretização formal de Crank-Nicolson:

$$
\left(\begin{array}{ccccc}
B & A & 0 & 0 & 0 \\
A & B & A & 0 & 0 \\
0 & A & B & A & 0 \\
0 & 0 & A & B & A \\
0 & 0 & 0 & A & B
\end{array}\right)\left(\begin{array}{c}
\vdots \\
\eta_{i-1} \\
\eta_{i} \\
\eta_{i+1} \\
\vdots
\end{array}\right)=\left(\begin{array}{ccccc}
B^{\prime} & A^{\prime} & 0 & 0 & 0 \\
A^{\prime} & B^{\prime} & A^{\prime} & 0 & 0 \\
0 & A^{\prime} & B^{\prime} & A^{\prime} & 0 \\
0 & 0 & A^{\prime} & B^{\prime} & A^{\prime} \\
0 & 0 & 0 & A^{\prime} & B^{\prime}
\end{array}\right)\left(\begin{array}{c}
\vdots \\
\xi_{i-1} \\
\xi_{i} \\
\xi_{i+1} \\
\vdots
\end{array}\right),
$$

na qual,

$$
A=\frac{-i \hbar \Delta t}{4 m \Delta x_{n}^{2}} \quad B=1+\frac{i \hbar \Delta t}{4 m \Delta x_{n}^{2}}
$$

e

$$
A^{\prime}=\frac{i \hbar \Delta t}{4 m \Delta x_{n}^{2}} \quad B^{\prime}=1-\frac{i \hbar \Delta t}{4 m \Delta x_{n}^{2}}
$$

Como os valores de $\xi_{i}$ está definido pela equação 13 , o produto indicado na parte direita da equação 17 pode ser feito diretamente. No entanto, o produto realizado no lado esquerdo deve ser solucionado através de uma rotina computacional conhecida como TRIDAG (PRESS et al., 2007).

Por fim, podemos definir a função de onda final aplicando a última exponencial envolvendo o potencial:

$$
\left|\psi_{i}\right\rangle_{t+\Delta t}=e^{-\frac{i}{2 \hbar} V_{i} \Delta t}\left|\eta_{i}\right\rangle_{t}
$$


Quando se é necessário estudar um sistema com mais de uma dimensão, baste repetir esse processo para cada uma das direções da energia cinética.

Para determinar os autoestados devemos utilizar a propagação no tempo imaginário. Pela ortogonalidade dos autoestados temos que uma base completa formada por eles, desta forma, podemos descrever uma função de onda como a combinação linear deste:

$$
\left|\psi>_{t}=\sum_{n=0}^{\infty} a_{n} e^{-\frac{i E_{n} t}{\hbar} \mathrm{V}_{i} \Delta t}\right| \phi_{n}>
$$

na qual $E_{n}$ e $\phi_{n}$ são as autoenergias e as autofunções respectivamente. Uma vez que a propagação é no tempo imaginário, consideraremos $\tau=i t$. Desta forma a equação 20 se tornará:

$$
\mid \psi>_{t}=e^{-\frac{E_{0} \tau}{\hbar}}\left[a_{0}\left|\phi_{0}>_{0}+\sum_{n=0}^{\infty} a_{n} e^{-\frac{\left(E_{n}-E_{0}\right) \tau}{\hbar} V_{i} \Delta t}\right| \phi_{n}>\right]
$$

Analisando $\tau$ vemos que quando essa função vai ao infinito o segundo termo tende a zero, obtendo, assim, o estado fundamental da função de onda e sua energia. Considerando que o estado fundamental é ortonormal ao primeiro estado excitado e consequentemente não fazendo parte de sua combinação linear, podemos obter o primeiro estado excitado ao propagar o tempo em direção ao infinito. Da mesma forma, fazemos para os próximos estados.

\section{RESULTADOS E DISCUSSÕES}

Foi calculado a energia do exciton em fios quânticos de Si/SiGe considerando dois tipos de alinhamento de banda, tipo-I e tipo-II, já que vários estudos experimentais mostram que dependendo da concentração de Ge na liga SiGe, esse material pode apresentar uma transição de alinhamento tipoI para tipo-II. Estes estudos mostram que para a fração molar de Ge até 0.25 na liga, o sistema tem alinhamento tipo-I, já para fração molar superior a 0.3 o alinhamento é do tipo-II.

A energia do exciton foi calculada variando alguns parâmetros tais como: raio do fio, interfaces graduais e campos magnéticos.

Na figura 3 é apresentado a energia do exciton do estado fundamental, formado pela ligação de um elétron e um buraco pesado (e- $h h$ ) em função do raio para um fio $\mathrm{Si} / \mathrm{Si}_{0.85} \mathrm{Ge}_{0.15}$ que apresenta alinhamento de banda do tipo I, considerando vários valores de espessura da interface: 0, 5, 10 e $15 \AA$. 
FIGURA 3 - Energia do estado fundamental do exciton e-hh em um fio quântico Si/Si ${ }_{0.85} \mathrm{Ge}_{0.15}$ tipo-I em função do raio do fio $(\rho)$, para espessuras da interface $w$ de $0 \AA$ (preta), $5 \AA$ (magenta), $10 \AA$ (azul) e $15 \AA$ (vermelha).

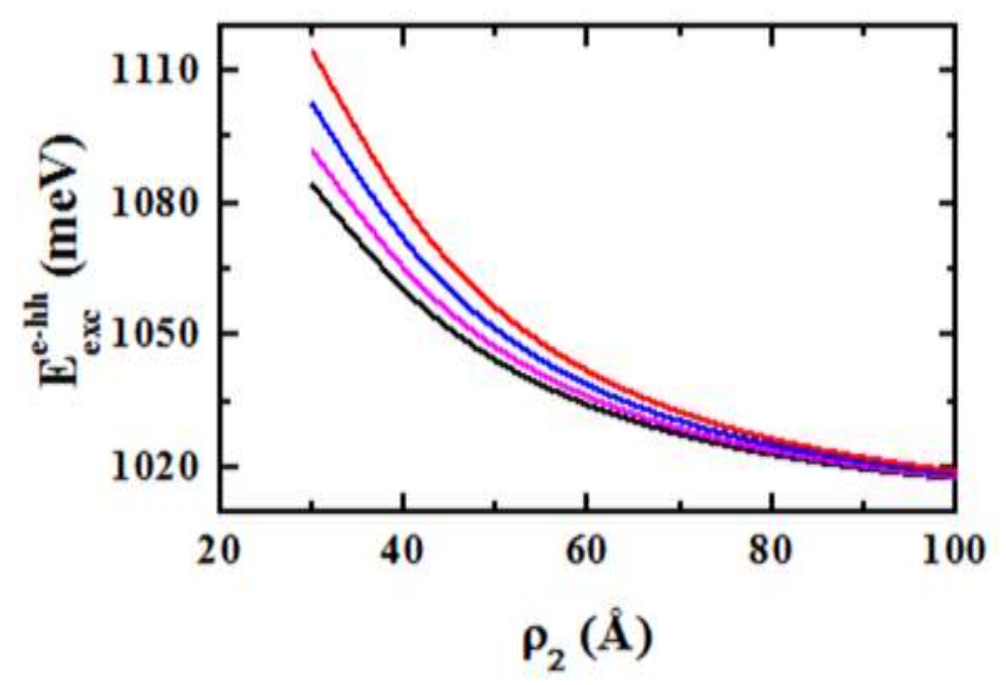

Fonte: Chaves, 2007 adaptado.

Pela figura 3 observa-se a energia diminui à medida que aumentamos o raio do fio e que as interfaces graduais realmente influenciam na energia, e essa influência é mais significativa para pequenos raios. Na figura 4 a energia de ligação do exciton é apresentado como uma função do raio para um fio $\mathrm{Si} / \mathrm{Si}_{0.85} \mathrm{Ge}_{0.15}$ para as mesmas condições anterior. É possível observar que a energia de ligação aumenta com o aumento do raio, até atingi o seu ponto máximo ( $\rho \approx 50 \AA$ ), após atingir esse máximo a energia passa a diminuir com o aumento do raio. Este comportamento se deve, pois, ao reduzir o raio do fio faz com que este sistema se assemelhe com um Si volumétrico, ou seja, um material bulk, no qual as energias de ligação são naturalmente mais baixas (CHAVES, 2007).

Ao observar a influência da interface gradual para valores de raios inferiores a $50 \AA$, temos que a energia diminui com o aumento da interface, já para raios superiores a $50 \AA$ observamos que o aumento da interface resulta no aumento da energia. E isso acontece novamente pelo fato de para pequenos raios teremos um material bulk. 
FIGURA 4 - Energia de ligação do exciton e-hh em um fio quântico Si/Si ${ }_{0.85} \mathrm{Ge}_{0.15}$ tipo-I em função do raio do fio ( $\rho)$, para espessuras da interface $w$ de $0 \AA$ (preta), $5 \AA$ (magenta), $10 \AA$ (azul) e $15 \AA$ (vermelha).

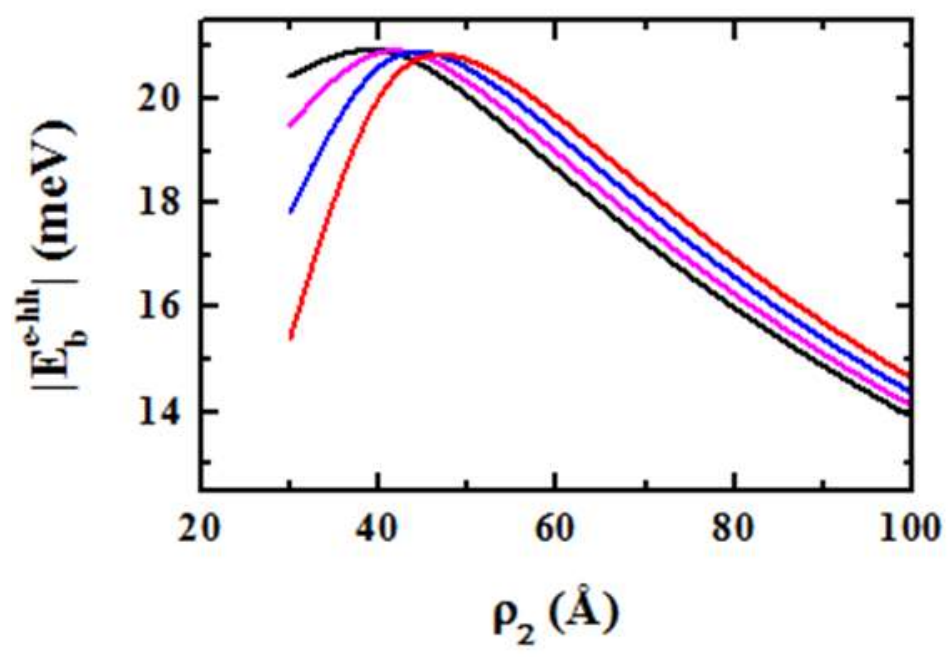

Fonte: Chaves, 2007 adaptado.

Na figura 5 a energia do estado fundamental do exciton é apresentada como uma função do raio para um fio Si/Si . $_{.70} \mathrm{Ge}_{0.30}$, para vários valores de espessura da interface $(0,5,10$ e $15 \AA$ ) considerando o alinhamento de banda do tipo II.

Pela figura 5 é possível perceber que quando o raio é aumentado há uma diminuição da energia, e que a presença da interface resulta no aumento da energia. No entanto quanto maior o raio do fio menor será a influência das interfaces graduais. 
FIGURA 5 - Energia do estado fundamental do exciton e-hh em um fio quântico $\mathrm{Si} / \mathrm{Si}_{0.70} \mathrm{Ge}_{0.30}$ tipo-Il em função do raio do fio $(\rho)$, para espessuras da interface $w$ de $0 \AA$ (preta), $5 \AA$ (magenta), $10 \AA$ (azul) e $15 \AA ̊$ (vermelha).

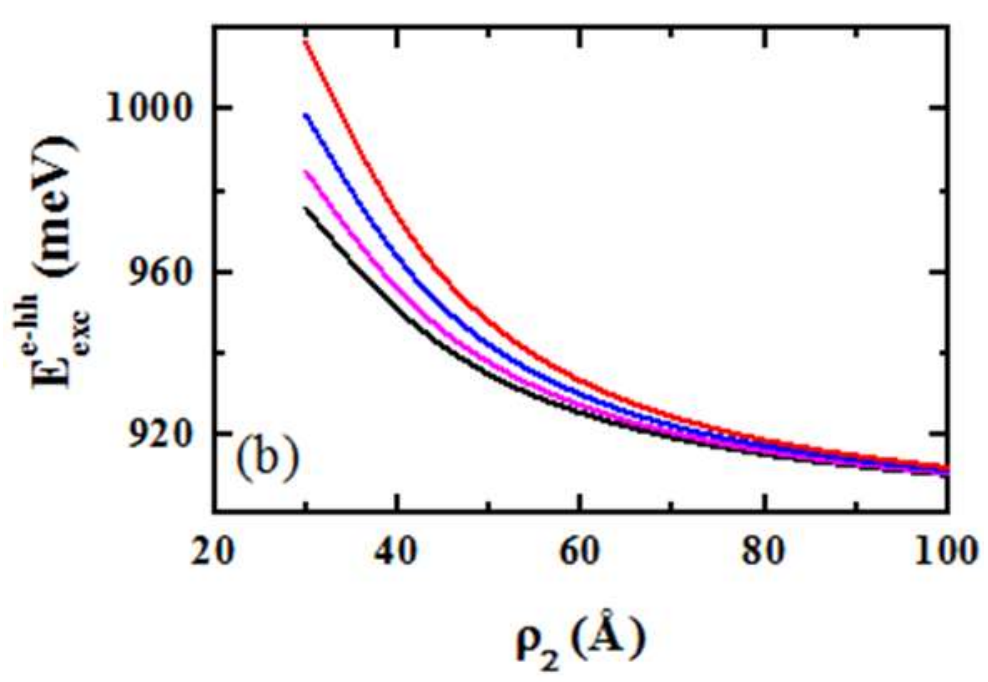

Fonte: Chaves, 2007 adaptado.

Na figura 6 é apresentada a energia de ligação do exciton (e-hh) como uma função do raio para um fio $\mathrm{Si} / \mathrm{Si}_{0,70} \mathrm{Ge}_{0,30}$ considerando as espessuras da interface de $0,5,10$ e $15 \AA$.

FIGURA 6 - Energia de ligação do exciton e-hh em um fio quântico Si/Si ${ }_{0.70} \mathrm{Ge}_{0.30}$ tipo-II em função do raio do fio ( $\rho)$, para espessuras da interface $w$ de $0 \AA$ (preta), $5 \AA$ (magenta), $10 \AA$ (azul) e $15 \AA$ (vermelha).

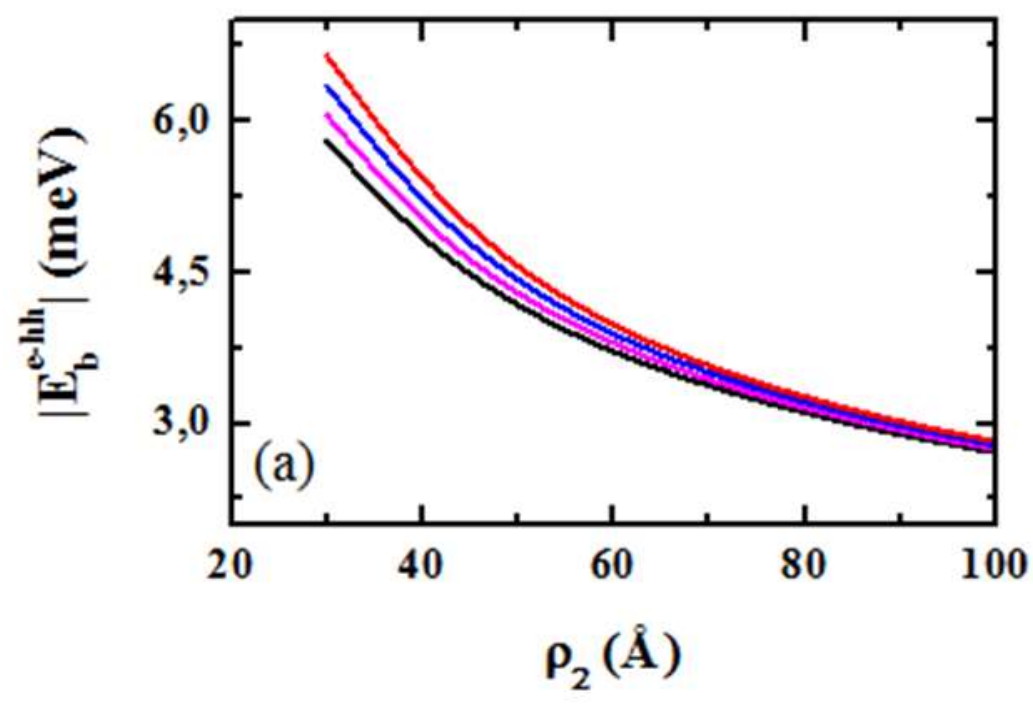

Fonte: Chaves, 2007 adaptado. 
Pela figura 6 é possível perceber que quando o raio é aumentado há uma diminuição da energia, e que a presença da interface resulta no aumento da energia. No entanto quanto maior o raio do fio menor será a influência das interfaces graduais.

Na energia de ligação para fios quânticos tipo-II, observa-se que da mesma forma que para tipo-I a energia é influenciada pela variação do raio e pela presença das interfaces graduais, no entanto agora a energia não aumenta para pequenos raios como aconteceu para tipo-I, uma vez que o buraco permanece confinado dentro de um fio fino e o elétron é ligado a ele na camada de Si o sistema não se parece com um Si volumétrico, e sim com um sistema volumétrico com uma impureza localizada (CHAVES, 2007).

Ao comprar as figuras 3 e 5 perceber-se que a energia do exciton é maior para estruturas de banda do tipo-I, pois nesta configuração tanto o elétron quanto o buraco estão confinados no mesmo material. Nas figuras 7 e 8 é apresentado a influência de um campo magnético aplicado paralelamente ao eixo de crescimento do fio, na energia de ligação e energia exciton, para uma interface abrupta (curvas) e interface de espessura $w=15 \AA$ (símbolos), para diferentes raios do fio, respectivamente.

FIGURA 7 - Energias de ligação do exciton e-hh em fios quânticos $\mathrm{Si} / \mathrm{Si}_{0,85} \mathrm{Ge}_{0,15}$ tipo-I como função do campo magnético, com $w=0 \AA$ (curvas) e $w=15 \AA$ (símbolos), para raios do fio $\left(\rho_{2}\right)$ de: $50 \AA$ (vermelha, $\Delta$ ), $100 \AA$ (verde, •), $150 \AA$ (azul, V) e $200 \AA$ (magenta, ت).

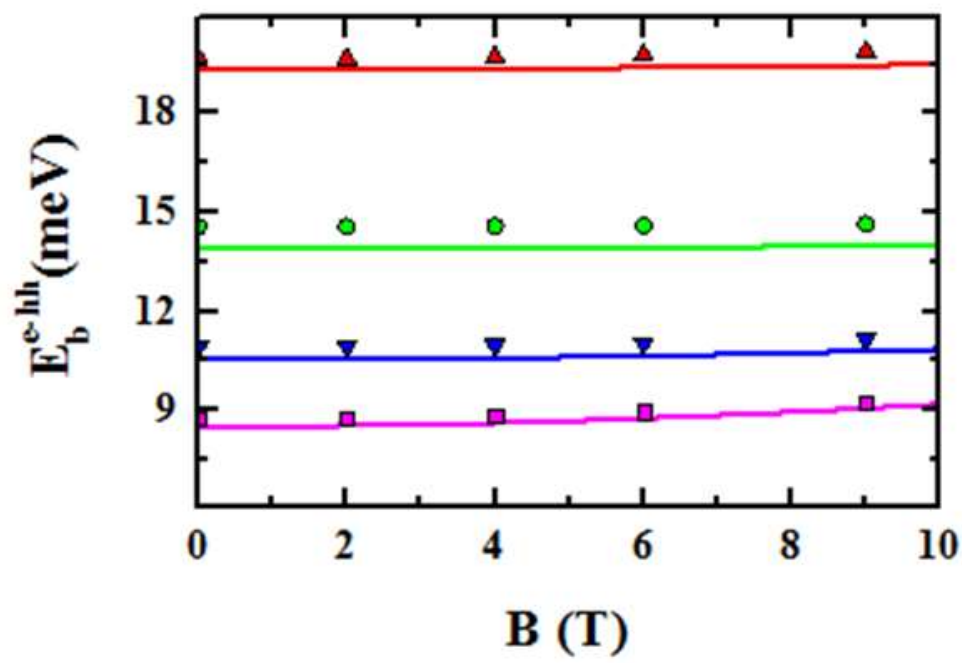

Fonte: Chaves, 2007 adaptado.

Os resultados apresentados nas figuras 7 e 8 evidenciam que o aumento do campo magnético não afeta consideravelmente estas energias, dando somente um pequeno acréscimo para todos os valores 
de raio quando consideramos a presença da interface de $15 \AA$ A. Além disso, percebe-se que as energias são maiores para raios menores.

FIGURA 8 - Energias de ligação do exciton e-hh em fios quânticos $\mathrm{Si} / \mathrm{Si}_{0,70} \mathrm{Ge}_{0,30}$ tipo-II como função do campo magnético, com $w=0 \AA$ (curvas) e $w=15 \AA$ (símbolos), para raios do fio $\left(\rho_{2}\right)$ de: $50 \AA$ (vermelha, $\triangle$ ), $100 \AA$ (verde, •), $150 \AA$ (azul, V) e $200 \AA$ (magenta, в).

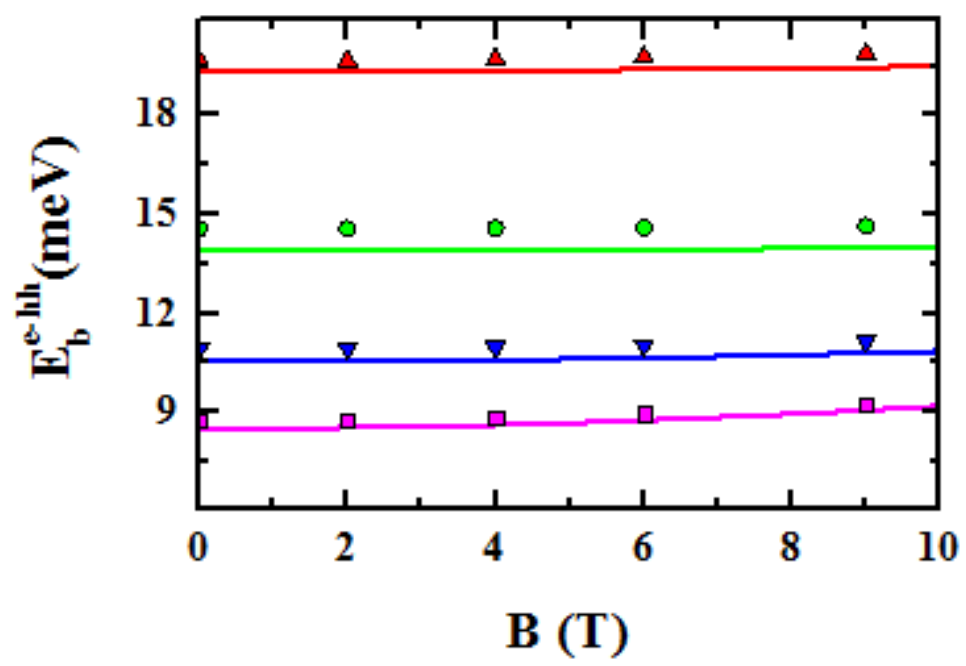

Fonte: Chaves, 2007 adaptado.

\section{PONTO QUÂNTICO}

Pontos quânticos são estruturas que confinam os portadores de carga nas três dimensões, resultando em uma forte quantização dos níveis de energia. Os pontos quânticos podem ser utilizados para simular átomos ou moléculas artificiais, permitindo manipular os portadores e sua energia de transição (SILVA, 2008).

\section{MÉTODO NUMÉRICO}

O ponto quântico estudado é um ponto com geometria elipsoidal, e será considerado o potencial Coulombiano para os sistemas tipo-I e tipo-II. Utilizando o modelo de evolução temporal Split-operator, que se baseia na solução da equação de Schrodinger dependente do tempo, para obter os níveis de energias. O Hamiltoniano pode ser escrito como:

$$
H=\frac{1}{2 m}(\vec{p}+q \vec{A})^{2}+V(\vec{r})+q \vec{F}(x),
$$

na qual $m$ representa a massa do elétron, $\vec{F}$ o campo elétrico aplicado ao longo do eixo $x$, o potencial vetor é um gauge simétrico do tipo: 


$$
\vec{A}=\frac{1}{2} \vec{B} \times \vec{\rho},
$$

e $V(\vec{r})$ o potencial total de confinamento, como, para o nosso caso, o potencial é suficiente para confinar o buraco, o confinamento do elétron é dado por:

$$
V(\vec{r})=V_{\text {het }}\left(\vec{r}_{e}, \vec{r}_{h}\right)+V_{\text {Coul }}\left(\vec{r}_{e}-\vec{r}_{h}\right),
$$

na qual:

$$
\begin{gathered}
V_{h e t}\left(\vec{r}_{e}, \vec{r}_{h}\right)=Q_{i}\left[\varepsilon_{1} \chi(z)+\varepsilon_{2} \chi^{2}(z)\right], \\
V_{\text {Coul }}\left(\vec{r}_{e}-\vec{r}_{h}\right)=\frac{-e^{2}}{4 \pi \sqrt{\left|\vec{r}_{e}-\vec{r}_{h}\right|^{2}}} .
\end{gathered}
$$

Utilizando a solução geral da equação Schrodinger

$$
\Psi(\vec{r}, t+\Delta t)=e^{\frac{-i H \Delta t}{\hbar}} \Psi(\vec{r}, t),
$$

substituindo o hamiltoniano da Equação 23 na equação 28, resultará numa exponencial com a soma dos termos cinéticos e potenciais, na qual esses termos não comutam. Para solucionar esse problema utilizaremos a expansão de Suzuki (SUZUKI, 1990), que consiste na decomposição das exponenciais dos operadores. Em seguida é utilizado uma função de onda tentativa, na forma de uma gaussiana, na qual é propagada no tempo imaginário $(t=-i r)$ até que a função de onda convirja para o estado fundamental do sistema, encontrando, por fim, os autoestados do sistema (SILVA, 2008).

\section{RESULTADOS E DISCUSSÕES}

O ponto estudado é composto dos materiais Si e SiGe formando a heteroestrutura Si/SiGe, e possui uma configuração elipsoidal com base circular. O estudo para o ponto foi dividido em duas partes: A primeira referente aos resultados para um ponto quântico com alinhamento do tipo-l e a segunda para o ponto com alinhamento do tipo-II;

Para o ponto com alinhamento tipo-I é considerado a composição de $15 \%$ de germânio, ou seja, $\mathrm{Si} / \mathrm{Si}_{0,85} \mathrm{Ge}_{0,15}$, na qual é calculado as energias dos portadores e a energia do estado fundamental do exciton (e-hh) variando o raio, para diferentes alturas.

A figura 9 mostra a energia do exciton no estado fundamental em função do raio, para as alturas de 15, 30, 45, 60, 75 e $90 \AA ̊$. Nessa figura é possível perceber a energia dos portadores não apresentam mudanças significativas com a variação do raio, no entanto quando variamos a altura do ponto, a variação nas energias é mais considerável. Para um ponto com raio de $150 \AA$ e altura de $15 \AA$ há um aumento de cerca de $80 \mathrm{meV}$ na sua energia em relação a um ponto de $90 \AA$. 
FIGURA 9 - Energia do exciton no estado fundamental em um ponto quântico elipsoidal de $\mathrm{Si} / \mathrm{Si}_{0.85} \mathrm{Ge}_{0.15}$ tipo-I em função do raio, para as alturas 15, 30, 45, 60, 75 e $90 \AA$.

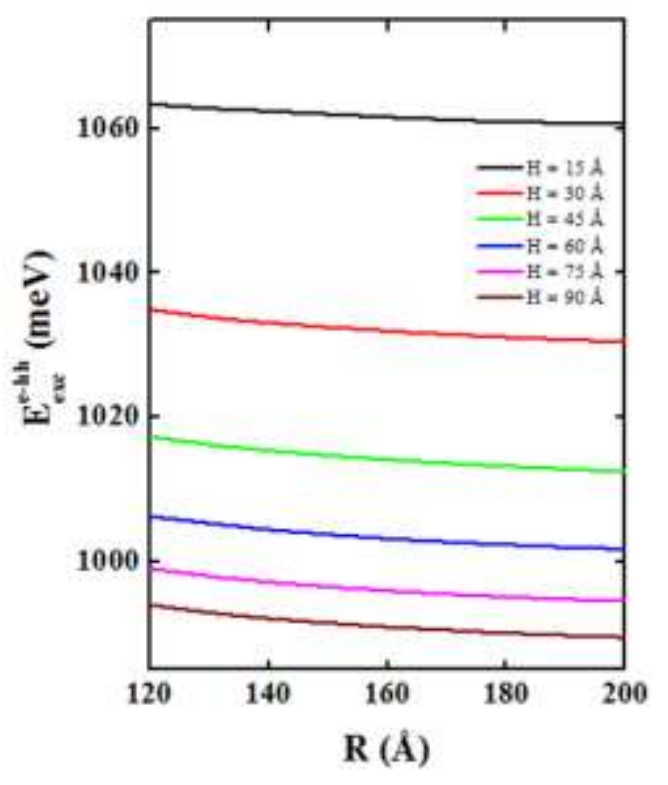

Fonte: Chaves, 2007 adaptado.

A figura 10 mostra a energia do buraco pesado em função do raio, para as alturas de 15, 30, 45, 60, 75 e $90 \AA$ A. Observa-se que para uma determinada altura a energia não tem mudanças significativas em relação ao raio, no entanto quando mudamos a altura do ponto vemos mudanças mais intensas nas energias. Mas isso é esperado, já que enquanto o raio varia de $120 \AA 200 \AA$, ou seja, um aumento de $40 \%$, as alturas variam bem mais, de $15 \AA$ a $90 \AA$, ou seja, um aumento de mais de $80 \%$. Para uma determinada altura $H=90 \AA$ com um raio de $150 \AA$ a diferença chega a aproximadamente $60 \mathrm{meV}$ em relação a uma altura de $H=15 \AA$. Como o referencial $V=0 \mathrm{meV}$ foi adotado sendo na barreira do $\mathrm{Si}$, as energias dentro do poço são negativas. 
FIGURA 10 - Energia do buraco pesado em um ponto quântico elipsoidal de $\mathrm{Si} / \mathrm{Si}_{0.85} \mathrm{Ge}_{0.15}$ tipo-I em função do raio, para as alturas 15, 30, 45, 60, 75 e $90 \AA$.

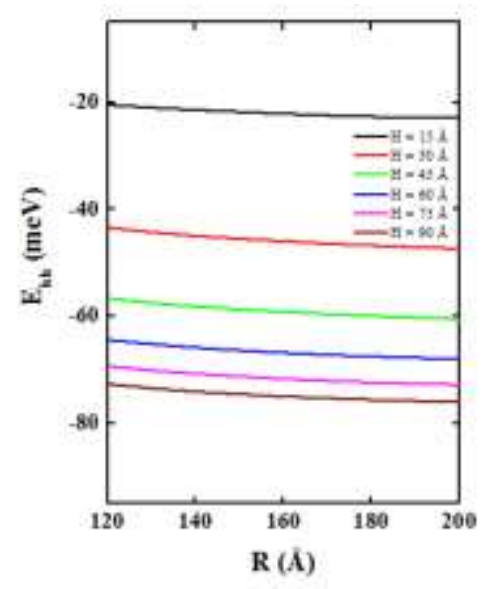

Fonte: Chaves, 2007 adaptado.

Para os resultados do tipo-Il foram calculadas a energia do estado fundamental do exciton (e-hh) e a energia do buraco pesado para uma variação de raio, considerando diferentes alturas.

Na figura 11 é apresentado a energia do exciton no estado fundamental em função do raio, para as alturas de $15,30,45,60,75$ e $90 \AA$. Nessa figura é possível perceber que assim como no caso do alinhamento tipo-I, a variação do raio não apresenta mudança significativa na energia, já a altura tem influência maior nas energias.

FIGURA 11 - Energia do exciton no estado fundamental em um ponto quântico elipsoidal de $\mathrm{Si} / \mathrm{Si}_{0.70} \mathrm{Ge}_{0.30}$ tipo-Il em função do raio, para as alturas $15,30,45,60,75$ e $90 \AA$.

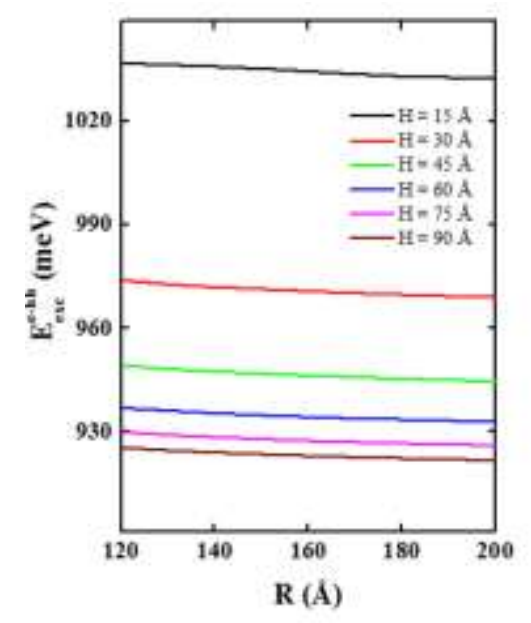

Fonte: Chaves, 2007 adaptado. 
Na figura 12 é apresentado a energia do buraco pesado em função do raio, para as alturas de 15, 30, 45, 60, 75 e $90 \AA ̊$. Essa estrutura apresenta mesmo comportamento que a anterior, praticamente não sofre influência do raio e sente mais a variação da altura.

FIGURA 12 - Energia do buraco pesado em um ponto quântico elipsoidal de $\mathrm{Si} / \mathrm{Si}_{0.85} \mathrm{Ge}_{0.15}$ tipo-I em função do raio, para as alturas 15, 30, 45, 60, 75 e $90 \AA$.

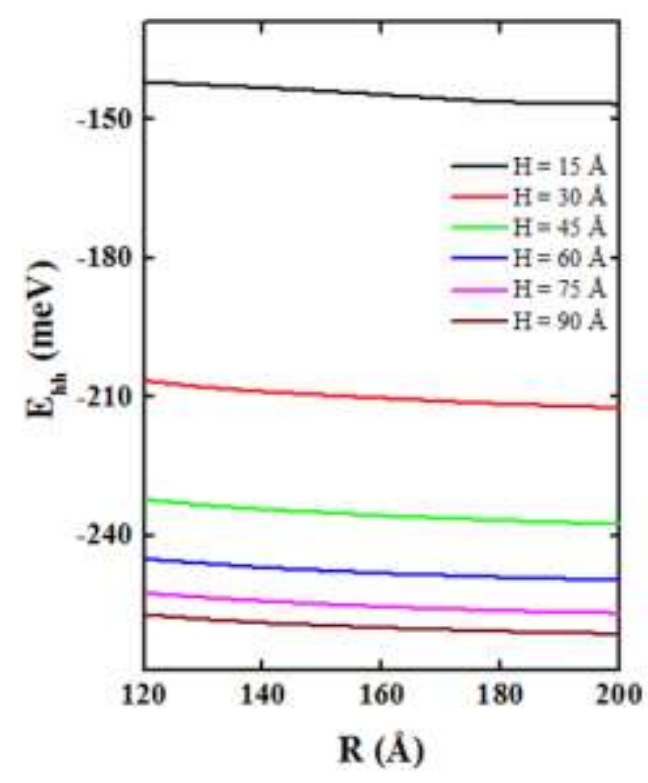

Fonte: Chaves, 2007 adaptado.

\section{CONCLUSÃO}

Neste trabalho foi realizado um estudo teórico acerca das propriedades eletrônicas em fios e pontos quânticos. $\mathrm{O}$ trabalho foi dividido em três partes. A primeira parte foi dedicada ao estudo bibliográfico, cuja finalidade foi de apresentar os conceitos básicos necessários para o entendimento das estruturas de baixa dimensão. A Segunda consistiu no estudo das propriedades em fios quânticos, para isso foram realizadas simulações numéricas baseadas no modelo de evolução temporal Split-operator. E a última, foi destinada ao estudo dos pontos quânticos, onde também foram realizadas simulações numéricas baseadas no Split-operator.

Para o estudo dos fios quânticos foram calculadas a energia de ligação e total do exciton, em função do raio para diferentes interfaces graduais e em função do campo magnético para diferentes raios e interfaces graduais. Para os alinhamentos de bandas do tipo-I ( $\mathrm{Si} / \mathrm{Si}_{0.85} \mathrm{Ge}_{0.15}$ ) e do alinhamento tipo-II (Si/Si $\mathrm{Si}_{0.70} \mathrm{Ge}_{0.30)}$.

As principais observações no estudo dos fios foram: 
- A influência das interfaces graduais na energia de ligação e total do exciton, tanto para estruturas do tipo-I quanto para o tipo-II;

- A influência do raio na energia de ligação e total do exciton, também para os dois tipos de alinhamento de banda;

- Comparando as energias total do exciton para o alinhamento do tipo-I e do tipo-II, observamos que a energia para tipo-I é maior que para o tipo-Il, e isso é justificado pois para tipo-I, ambos os portadores estão confinados;

- A pouco ou nenhuma influência do campo magnético na energia do exciton e na energia de ligação do exciton, independentemente do raio do fio ou da interface gradual.

No estudo dos pontos quânticos foram calculadas a energia do estado fundamental do exciton e a energia do buraco pesado em função do raio para diferentes interfaces graduais. Para os alinhamentos de bandas do tipo-I ( $\mathrm{Si} / \mathrm{Si}_{0.85} \mathrm{Ge}_{0.15}$ ) e do alinhamento tipo-II (Si/Si $\mathrm{Si}_{0.70} \mathrm{Ge}_{0.30}$ ).

As principais observações no estudo dos pontos foram:

- A influência da variação do raio nos níveis de energia e na energia total do exciton, que apesar de pequena, resulta na diminuição destas energias, independentemente do tipo de alinhamento de banda;

- A influência da altura do ponto quântico nos níveis de energia do buraco pesado e na energia total do éxciton, onde observa-se que essas energias estão susceptíveis a mudança da altura, independentemente do tipo de alinhamento de banda;

- Comparando os gráficos da energia do exciton para os diferentes alinhamentos de bandas, assim como no fio quântico, observamos que a energia será maior para tipo-I, uma vez que nele os dois portadores de carga estarão confinados. 


\section{Capítulo 4}

\section{d.)}

\section{ÁLGEBRAS ESTANDARMENTE ESTRATIFICADAS: ÁLGEBRAS DE NAKAYAMA}

Fidel Hernández Advíncula
Facultad de Matemática y Computación

Universidad de La Habana, Cuba 
Resumo: Neste trabalho estudamos as álgebras estandarmente estratificadas.

Mostramos alguns exemplos. Por último façemos um analises da estratificação de certas álgebras de Nakayama.

Palavras chaves: Módulos filtrados, álgebras estandarmente estratificadas, álgebras de Nakayama. 


\section{INTRODUÇÃO}

Começamos com os módulos estandar e suas propiedades, posteriormente de_nimos as _algebras estandarmente estratificadas, olhamos algums exemplos.

A continuação introducimos as álgebras de Nakayama e suas propiedades e fazemos um analisis da estratificação de certas álgebras de Nakayama.

\section{MÓDULOS ESTANDAR. ÁLGEBRAS ESTANDARMENTE ESTRATIFICADAS}

Neste trabalho todas as álgebras são K- álgebras de dimensão finita, básica e indecomponível um corpo algebricamente fechado, e por um teorema fundamental de Gabriel toda álgebra $\Lambda$ com estas propriedades é da forma ${ }^{\Lambda=\frac{K Q}{l}}$ onde $Q$ é um quiver finito e $I_{\text {um ideal admissível. }}$

Sejam $v_{1} \ldots v_{n}$ os vértices de $Q$ numa ordem fixada e $S 1 ; \ldots S_{n}$ a ordenação correspondente dos módulos simples, $\mathrm{P}_{\mathrm{i}}$ a cobertura projetiva de Si. Define-se o módulo estandar $\Delta_{i}$ como o quociente máximo de Picom fatores de composição $S_{j}, j \leq i$.

Seja $\Delta=\left\{\Delta_{1}, \ldots, \Delta_{n}\right\}$, consideremos $F(\Delta)$, a subcategoria plena de $\bmod \wedge$; formada pelos $M \in \bmod \wedge$ tais que $\mathrm{M}$ tem uma filtração com quocientes em $\Delta_{\text {seja, }}$ $0=M_{0} \subset M_{1} \subset \ldots \subset M_{t}=M \operatorname{com} \frac{M_{i}}{M_{i-1}} \simeq \Delta_{k}$.

Definição 1 A álgebra $\Lambda$ chama-se estandarmente estratificada se $\Lambda \in F(\Delta)$, Se além disso, o anel de endomorfismos de cada módulo estandar é simples $\Lambda$ diz-se quase hereditária.

Dualmente define-se o módulo coestandar $\nabla_{i}$ como o submódulo máximo de ${ }^{Q_{i}}$ com fatores de composição $S_{j}, j \leq i$. onde $Q_{i}$ é a envolvente injetiva de $S_{\text {i. }}$

Dualmente define-se álgebra $\Lambda$ coestandarmente estratificada se $\Lambda \in F(\nabla)$.

\section{EXEMPLOS E PROPIEDADES}

Nos exemplos seguentes vemos que o conceito de álgebra $\Lambda$ estandarmente estratificada depende da ordem dos simples. 
Exemplo 2 Seja A álgebra de carcaz dada pelo carcaz seguinte

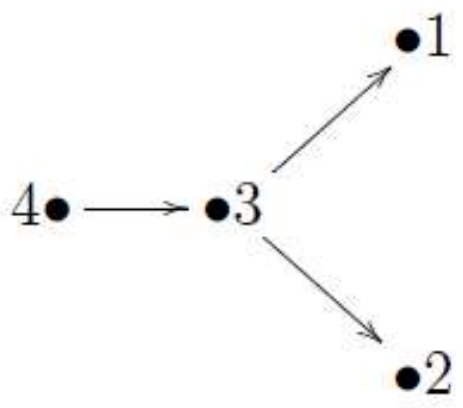

3

com esta ordem $P_{1}=S_{1}, P_{2}=S_{2}, P_{3}$

1

2

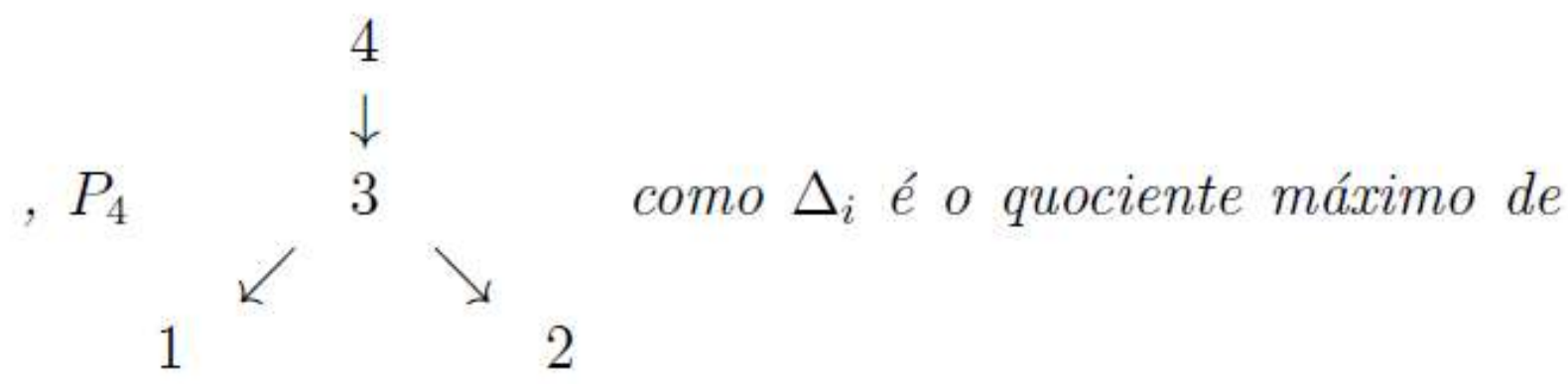

$P_{i}$ com fatores de composição $S_{j}, j \leq i_{\text {temos que }} \Delta_{1}=P_{1}, \Delta_{2}=P_{2}$, de uma qualquer das dois series de composição de $\mathrm{P}_{3}$ :

$0 \subset S_{1} \subset \operatorname{radP}_{3} \subset P_{3}$ ou $0 \subset S_{2} \subset \operatorname{radP}_{3} \subset P_{3}$ temos que $\Delta_{3}=P_{3}$ e analogamente $\Delta_{4}=P_{4}$.

Neste caso todos os $\Delta_{i}=P_{i}$, quando isso suceder $F(\Delta)=\operatorname{Proj} A$.

Proposição $3 F(\Delta)=\operatorname{Proj} A \Leftrightarrow \operatorname{Todo} \Delta_{i}$ é projetivo.

Prova. Se $F(\Delta)=\operatorname{Proj} A$, então é claro que todo $\Delta_{i}$ é projetivo pois todo $\Delta_{i} \in F(\Delta)$.

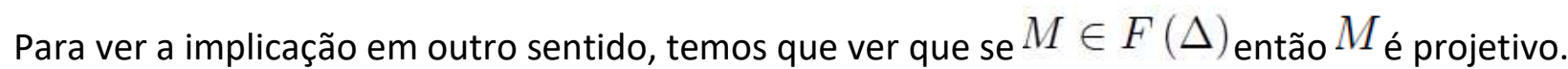


Façamos indução em $l(M)$ o comprimento de ${ }^{M}$.

Para $M=\Delta_{i}$ claro.

Seja agora $M \in F(\Delta)_{\text {temos }} 0=M_{0} \subset M_{1} \subset \ldots \subset M_{t}=M \operatorname{com} \frac{M_{i}}{M_{i-1}} \simeq \Delta_{k}$ que é projetivo assim temos $0 \rightarrow N \rightarrow M \rightarrow \Delta_{i} \rightarrow 0$, esta sequência cinde, ou seja $M \simeq$ $\Delta_{i} \oplus N$, mas $N_{\text {tem comprimento menor que }} M_{\text {,logo é projetivo e }} \Delta_{i}$ é projetivo, então $M$ é projetivo.

\section{Exemplo 4}

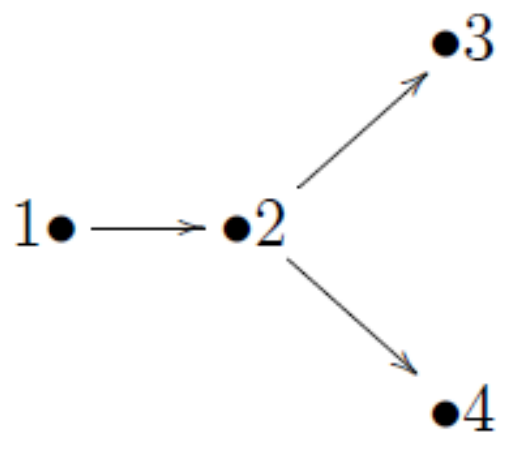

este é o carcaz do exemplo anterior com outra ordenação dos simples, aqui facilmente vemos que $\Delta_{i}=S_{i}$ para todo ${ }^{i}$, neste caso claro que $F(\Delta)=\bmod A$.

Proposição $5(\Delta)=\bmod A \Leftrightarrow \Delta_{i}=S_{i}$.

Prova.

Se $F(\Delta)=\bmod A$,então claro que $S_{i}, \forall i, \operatorname{logo}$ todo $S_{i}$ tem uma filtração em $\Delta_{\text {,como é simples }}$ $S_{i}=\Delta_{i}$

Se $S_{i}=\Delta_{i}$ como os módulos que tem filtração em todos os simples são todos os $A$ módulos, então $F(\Delta)=\bmod A$.

Em geral $\mathcal{F}(\Delta) \subset P^{<\infty}[7]$. 


\section{Exemplo 6}

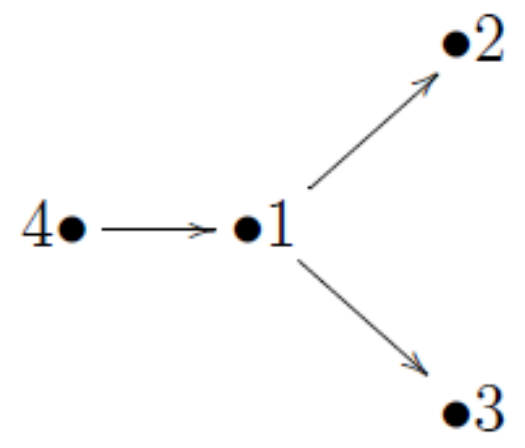

este é o carcaz dos exemplos anteriores com outra ordenação dos simples, neste caso claro que $\Delta_{1}=S_{1}, \Delta_{2}=\begin{aligned} & S_{2}, \Delta_{3}=S_{3} \text { e } \Delta_{4}=P_{4}, \operatorname{logo} F(\Delta) \neq \operatorname{Proj} A \text { e } F(\Delta) \neq \\ & \bmod A .\end{aligned}$ A seguinte proposição sera útil na seguinte seção.

Proposição 7 Se para uma álgebra $\wedge$ todo quociente de projectivos indescomponibles tem dimensão projectiva infinita, então $\wedge$ não é estandarmente estratificada en nenhuma ordem dos simples.

Prova. Claramente satisface-se que $\Delta_{i} \in \mathcal{F}(\Delta)$, além disso cada $\Delta_{i}$ é quociente do projectivo $P_{i}$. Como assumimos que todo quociente de projectivos indescomponibles tem dimensión proyectiva infinita, verifica-se então que $p \boldsymbol{d} \Delta_{i}=\infty \operatorname{logo} \mathcal{F}(\Delta)$ não pode estar conteúdo em $P^{<\infty}$ e em consequência $\wedge$ não é estandarmente estratificada em nenhuma ordem dos simples.

Exemplo 8 Seja $\wedge$ a álgebra dada pelo sieguiente carcaz, com o ideal $I=\langle\beta \alpha, \alpha \gamma \beta, \alpha \gamma\rangle$;

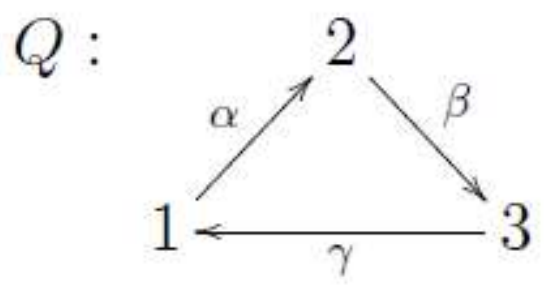

Temos que: 


$$
P_{1}: \begin{array}{rr}
1 \\
2
\end{array} \quad P_{2}: \begin{array}{r}
2 \\
3 \\
1
\end{array} \quad P_{3}: \begin{aligned}
& 3 \\
& 1
\end{aligned} \quad \text { e } \quad I_{1}: \begin{aligned}
& 2 \\
& 3 \\
& 1
\end{aligned} \quad I_{2}: \begin{aligned}
& 1 \\
& 2
\end{aligned}
$$

$I_{3}: \begin{aligned} & 2 \\ & 3\end{aligned}$

Podemos comprovar que esta álgebra é estandarmente estratificada y coestandarmente estratificada únicamente para as respeitivas ordens dos simples dados a continuação,

1. Para a ordem $2,1,3 \rightsquigarrow \Delta=\left\{\mathcal{S}_{2}, \mathcal{P}_{1}, \mathcal{P}_{3}\right\} \quad$ todos os $P_{i}$ estão filtrados.

2. Para a ordem $1,2,3 \rightsquigarrow \nabla=\left\{\mathcal{S}_{1}, \mathcal{I}_{2}, \mathcal{I}_{3}\right\}$ todos os $I_{i}$ estấo filtrados.

\section{4 ÁLGEBRAS DE NAKAYAMA}

Nesta seção presentaremos as Álgebras de Nakayama as quais aplicaremos a teoría exposta nas seções anteriores.

Definição 9 Seja $M$ un $\wedge$ - módulo. Define-se a serie radical de $M$ do modo seguinte:

$$
0 \subset \ldots \subset \operatorname{rad}^{2} M \subset \operatorname{rad} M \subset M
$$

Denotaremos por $r l(M)$ o comprimento da série radical de $M$.

Dualmente podemos definir a serie soclo de $M$. 


$$
\begin{gathered}
\operatorname{soc}^{0} M:=0 \\
\operatorname{soc}^{i+1} M:=\pi^{-1} \operatorname{soc}\left(\frac{M}{\operatorname{soc}^{i} M}\right)
\end{gathered}
$$

onde $\pi: M \rightarrow \frac{M}{\operatorname{soc}^{i} M}$ é a aplicação quociente, isto é,

$$
\frac{\operatorname{soc}^{i+1} M}{\operatorname{soc}^{i} M} \cong \operatorname{soc}\left(\frac{M}{\operatorname{soc}^{i} M}\right) \text {. }
$$

Denotaremos por $\operatorname{sl}(M)_{\text {o comprimento da série soclo de }} M$.

Notemos que para $M \neq 0, \operatorname{rad}_{\text {é submódulo de } M \text { e além disso }} \operatorname{dim}_{k} M<\infty$, isto implica que a serie radical de $M$ é finita. Vejamos também que $\operatorname{rad}^{i} M=(\operatorname{rad} \Lambda)^{i} M$, de aqui temos que $\operatorname{rad}^{i} \Lambda=(\operatorname{rad} \Lambda)^{i}$ e $r l(M) \leq r l(\Lambda)$.

Podemos notar também que $\operatorname{dim}_{k} M<\infty, \log \operatorname{soc} M \neq 0$ si $M \neq 0$ e a série do soclo.

$$
0 \subset \operatorname{soc} M \subset \operatorname{soc}^{2} M \subset \ldots \subset M
$$

Es finita.

Proposição 10 Seja $M \in \bmod \Lambda, \operatorname{sl}(M)=\operatorname{rl}(M)$.

Dentição 11 Definimos o comprimento de Loewy $\|(M):=r l(M)=s l(M)$.

Temos que introducir novas noções para definir as Álgebras de Nakayama.

Definição 12 Seja $M$ um $\Lambda$ - módulo. Dizemos que $M$ es uniserial se posee uma única serie de composição.

Lema 13 Seja M un ^- módulo. As seguientes condições são equivalentes.

1. M é uniserial 
2. A serie radical de $M$ é uma serie de composição.

3. La serie soclo de M é uma serie de composição.

4. $l(M)=l l(M)$

Dentição 14 Dizemos que uma álgebra $\wedge$ é serial direita (respeitivamente esquerda) se todo projectivo indescomponible direito (respeitivamente esquerdo) é um $\Lambda$ - módulo uniserial.

O seguiente Teorema permite caracterizar as álgebras básicas seriais a partir de seu carcaz subjacente.

Teorema 15 Uma K-_álgebra básica é serial esquerda se e so se para cada vértice $\alpha$ no carcaz

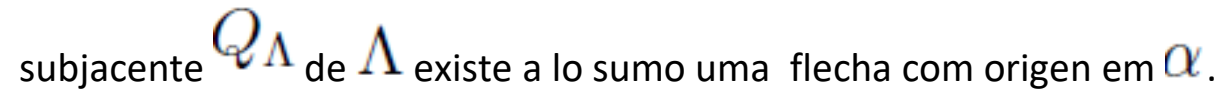

Corolário 16 Uma K- álgebra básica é serial direita se e so se para cada vértice _ no carcaz subjacente $Q_{\Lambda}$ de $\wedge$ existe $a$ lo sumo uma echa que termina em $\alpha$.

Temos agora os recursos necessários para definir as Álgebras de Nakayama.

Definição 17 Dizemos que uma álgebra $\Lambda$ é de Nakayama se é simultaneamente serial direita e serial esquerda, isto é, os projectivos e os injectivos indescomponibles são uniseriales.

Podemos caracterizar as Álgebras de Nakayama mediante seu carcaz subjacente.

Teorema 18 Uma álgebra básica e conexa é uma Álgebra de Nakayama se e so se o carcaz subjacente $Q_{\Lambda}$ é de umaa das duas formas seguientes:

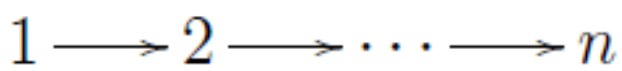

$O$

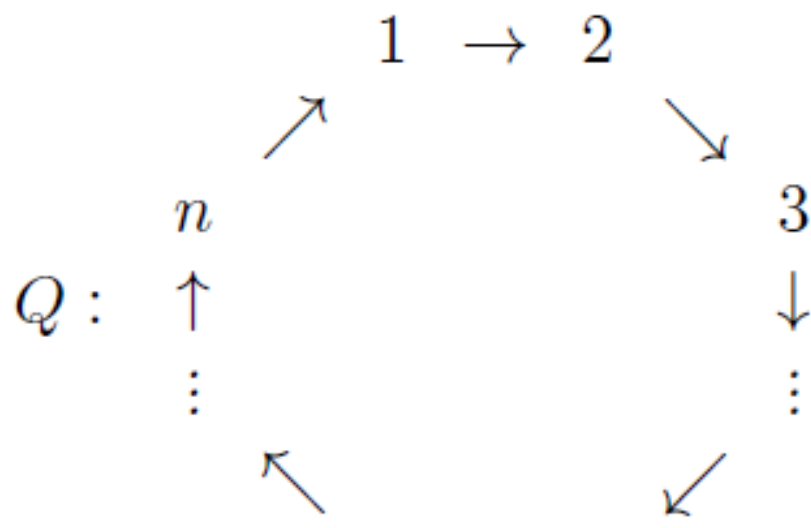


Prova.

Consequência do Teorema 15 y do Corolário 16.

\section{RESULTADO PRINCIPAL}

Dentro das_álgebras de Nakayama prestaremos atenção a aquelas cujo carcaz subjacente $Q_{\Lambda}$ é do tipo cíclico e com a relação $\operatorname{rad}^{r} K Q=0$. Denotaremos estas álgebras por $\Lambda_{n}^{r}$.

Teorema 19 Não existe nenhuma ordem dos simples taql ue alguma das álgebras do tipo $\Lambda_{n}^{r}$ seja estandarmente estratificada o coestandarmente estratificada.

Prova. E fácil ver que todos os $P_{i}$ tem o mesmo comprimento e sabemos ainda que poseen uma única serie de composición. Seja $\mathrm{M}$ um quociente de $P_{i}$ e consideremos a sequencia exacta curta seguiente,

$$
0 \longrightarrow M_{1} \longrightarrow P_{i} \longrightarrow M \longrightarrow 0
$$

Observemos que o comprimento do $\Lambda$ - módulo $\mathrm{M}_{1}$ é menor estricto que o de $P_{i}$, de onde podemos afirmar que $M_{1}$ não é projectivo. Consideremos agora a seguiente sequência exacta curta,

$$
0 \longrightarrow M_{2} \longrightarrow P_{i}^{1} \longrightarrow M_{1} \longrightarrow 0
$$

Aqui, novamente, observamos que o comprimento do $\wedge$ - módulo $\mathrm{M}_{2}$ é menor estricto que o de $P_{i}^{1}$, de onde podemos afirmar que $M_{2}$ não é projectivo. Sucesivamente é posible aplicar este razonamiento, de onde obtemos uma resolução projectiva minimal de $\mathrm{M}$ infinita. Así podemos afirrmar que $p d M=\infty$. Agora como $\mathcal{F}(\Delta) \subset P^{<\infty}$ tem-se resultado.

Em general as álgebras de Nakayama que não são $\Lambda_{n}^{r}$ podem ser o não estandarmente estratificadas, como podemos apreciar no exemplo 8. 


\section{CONCLUSÕES}

Neste trabalho temos estudado as álgebras estandarmente estratificadas, alumas de suas propiedades e exemplos.

Também temos visto as álgebras de Nakayama e temos feito un analisis da estratificação das mismas, mostrando que as álgebras de Nakayama do tipo $\Lambda_{n}^{r}$, não podem ser estandarmente estratificadas em nenhuma ordem dos simples.

Um problema aberto sería intentar realizar o mismo analisis de estratificação para outras álgebras de Nakayama não cíclicas. E 


\section{REFERÊNCIAS}

[1] F.W. Anderson and K. R. Fuller, Rings and Categories of Modules, Graduate texts in Mathematics, 13, Springer-Verlag, Heildeberg-Berlin, 1973.

[2] V. Dlab, C.M. Ringel. Auslander Algebras as Quasi- Hereditary Agebras. Department of Mathematics and Statistics, Carleton University and Fakultat fur Mathematik, Universitat Bielefeld, pp: 1 ? 10, 1991.

[3] J.F. Hernandez. Sobre as álgebras estandarmente estraticadas Tese de doutorado,Instituto de Matemática e Estatística da Universidade de São Paulo, Brasil, 2004.

[4] J.F. Hernandez,R.F. Ochoa. Stratication Analysis of Certain Nakayama Algebras Advances in Pure Mathematics, Vol. 5, No. 14, 2015, pp:850?855:; 2004.

[5] T. Nakayama Note on Uni-serial and Generalized Uniserial Rings. Mathematical Institute, Osaka Imperial University, Japon, pp: 285 e 2891940.

[6] R.F. Ochoa. Analisis de Estraticacion para ciertas Algebras de Nakayama Tesis de maestra, Facultad de Matemática y Computación, Universidad de la Habana, Cuba, pp: 1 -54, 2014.

[7] M. I. Platzeck, I.Reiten, Modules of nite projective dimension for standardly stratied algebras,

Communications in Algebra, Vol. 29, No.3, pp. 973-986, 2001.

[8] G. Sisodia. Nakayama Algebras, 2011. Artculo, Universidad de Sao Paulo, Brasil, pp: 1 - 8, 2007.

[9] R. Tan Auslander Algebras of Self-Injective Nakayama Algebras Department of Mathematics, Hubei University, P.R. China, pp: 89 - 99, 2013. 


\section{Capítulo 5}

d.)

\section{ESTABILIZAÇÃO POR REALIMENTAÇÃO DE SAÍDA NO POSICIONAMENTO REGIONAL DE PÓLOS}

Elmer Rolando Llanos Villarreal

Walter Martins Rodriuges

Samara Jessica Monte Bezerra

Jose Alfredo Ruiz Vargas
Universidade Federal Rural do Semi-Árido

Universidade Federal Rural do Semi-Árido

Universidade Federal Rural do Semi-Árido

Universidade de Brasília 
Resumo: O artigo base foi apresentado no 10 Congresso Brasileiro de Controle de Dinâmica e suas Aplicações (DINCON 2011), foi realizada uma revisão do artigo que agora será um capítulo de livro. 0 presente artigo considera o problema de estabilização para realimentação de saída estático por posicionamento regional de polos em desigualdades de matrizes lineares, regiões (LMIs). Uma nova abordagem para o projeto do controlador de realimentação de saída é proposta e os respectivos ganhos de realimentação de saída são obtidos através da solução da equação de Sylvester acoplada que está relacionada á solução das equações de Lyapunov que permitem obter a colocação de polos em LMI regiões do plano complexo. Ele apresentou condições necessárias e suficientes para a existência da matriz estática de realimentação de saída. Este artigo também propõe um novo algoritmo de solução numericamente e ciente para as equações de Sylvester acoplado para determinar a matriz de realimentação de saída onde a solução pode ser obtida usando a condição $(m+p>n)$.

Palavras Chave: Estabilização, Realimentação de saída, sistemas descritores. 


\section{INTRODUÇÃO}

O problema de realimentação de saída estático por posicionamento regional de polos em um sistema linear, invariante no tempo e multivariável usando realimentação de saída é considerado neste artigo. A estabilização de sistemas lineares por realimentação de saída é reconhecida como um problema importante e ainda em aberto na teoria de controle. Para sistemas normais, abordagens e técnicas para tratar diferentes versões deste problema, ele pode ser encontrado em [5], [13]. Abordagens e técnicas para tratar sistemas descritores por feedback de saída podem ser encontradas em [17], [18]. Muitos problemas em sistemas de teoria de controle são resolvidos quando calculada a solução das equações de Sylvester. Essas equações têm aplicações importantes na análise de estabilidade e na atribuição de autovalores (ver [15]; [1]; [5]). Existem condições necessárias e suficientes para a solução dessas equações em [5]. A Solução da equação de Sylvester associada a um sistema descritor linear em [4], [10]. Neste artigo, uma abordagem de realimentação de saída e um algoritmo são apresentados para calcular soluções por meio de técnicas LMI.

A solução das equações de Sylvester está relacionada com a solução das equações de Lyapunov que permitem obter a colocação dos polos nas regiões LMI. Assim, são apresentadas as condições necessárias e suficientes para a existência da matriz de realimentação de saída estática na colocação dos polos. Este artigo também propõe um algoritmo de solução numericamente eficiente para as equações de Lyapunov acopladas para determinar a matriz de realimentação de saída. Finalmente, muitas condições teóricas foram oferecidas para a existência de matriz de realimentação de saída.

Este artigo está organizado da seguinte forma. A segunda seção apresenta o problema com base no básico conceitos e conceitos básicos de regiões de LMI. Na seção 3, apresentamos alguns resultados na forma de equações de Sylvester, o Teorema para a existência da matriz de feedback de saída. Exemplos numéricos ilustram o aplicação dos algoritmos que descrevem as etapas básicas usado para resolver o problema. Eles são apresentados na quarta seção. Por fim, são apresentadas as considerações finais.

\section{PRELIMINARES}

As soluções para a estabilização do problema estão associadas _a existência de um subespaço $\mathcal{V}=\operatorname{Ker}(T)(C, A, B)$ invariante fortemente estabilizante, devido á possibilidade de resolver o equações de Sylvester acopladas, reescritas abaixo: 


$$
\begin{aligned}
A V+V H_{V} & =-B W \text { com } \sigma\left(H_{V}\right) \in \mathcal{C}^{-} \\
T A+H_{T} T & =-U C \text { com } \sigma\left(H_{T}\right) \in \mathcal{C}^{-} \\
T V & =0 \\
\operatorname{Ker}(C V) & \subseteq \operatorname{Ker}(W) \\
\operatorname{Ker}\left(B^{\prime} T^{\prime}\right) & \subseteq \operatorname{Ker}\left(U^{\prime}\right)
\end{aligned}
$$

Em [5], as equações acopladas de Lyapunov também podem ser usadas para descrever propriedades geométricas necessárias para resolver o problema de estabilização.

Teorema 2.1. : [5] Existe uma matriz de realimentação de saída $G \in \Re^{m \times p}$ tal que $\sigma(A+B G C) \in \mathcal{C}^{-}$, se e somente se as condições veri_cadas para algumas matrizes de posto completo $V \in \Re^{n \times v}$ e $T \in \Re^{n-v \times n}$ com inteiro $0<v \leq n$, tal que $T V=0$ : (i) $\forall Q_{V}=Q_{V}^{\prime}>0, Q_{V} \in \Re^{v \times v}$, existem matrizes $P=P^{\prime} \geq 0, P \in \Re^{n \times n} e$ $Y \in \Re^{m \times n}$ tal que :

$$
\begin{array}{r}
A P+P A^{\prime}+B Y+Y^{\prime} B^{\prime}=-V Q_{V} V^{\prime} \\
V^{\prime} P V>0 ; T P T^{\prime}=0 \\
Y=W_{\Pi} V^{\prime} \text { para alguns } W_{\Pi} \in \Re^{m \times v}
\end{array}
$$

(ii) $\forall Q_{T}=Q_{T}^{\prime}>0, Q_{T} \in \Re^{n-v \times n-v}$ existem as matrizes $S=S^{\prime} \geq 0, S \in \Re^{n \times n}$ and $Z \in \Re^{n \times v}$ tal que:

$$
\begin{array}{r}
A^{\prime} S+S A+C^{\prime} Z^{\prime}+Z C=-T^{\prime} Q_{T} T \\
T S T^{\prime}>0 ; \quad V^{\prime} S V=0 \\
Z=T^{\prime} U_{\Gamma} \text { para alguns } U_{\Gamma} \in \Re^{n-v \times v}
\end{array}
$$

(iii)

$$
\begin{array}{r}
\operatorname{Ker} C P \subseteq \operatorname{Ker} Y \\
\operatorname{Ker} B^{\prime} S \subseteq \operatorname{Ker} Z^{\prime}
\end{array}
$$




\section{POSICIONAMENTO REGIONAL DE PÓLO}

Uma solução para o problema de estabilização para obter a matriz de feedback de saída foi baseada na colocação arbitraria de pólos. Existem soluções para o problema de estabilização considerando o deslocamento de $\alpha$ usando $(A+\alpha I)$ ) em vez de A.

A possibilidade de estabilizar e situar os pólos em uma determinada region LMI D como em [9].

Seja $\mathcal{D} \subseteq \mathcal{C}^{-}$alguma região do plano complexo. O problema de estabilização é encontrar uma matriz de realimentação de saída $u=G y$ que tal $\sigma(A+B G C) \in \mathcal{D}$, ou equivalentemente, o sistema de malha fechada é em D - estável.

Um $\operatorname{par}(A, B)$ é $\mathcal{D}$ - estabilizável se e somente se

$$
\text { posto }\left(\left[\begin{array}{ll}
\lambda I-A & B
\end{array}\right]\right)=n, \forall \lambda \notin \mathcal{D}
$$

e um par $(C, A)$ é $\mathcal{D}$-detectável se e somente se

$$
\text { posto }\left(\left[\begin{array}{c}
\lambda I-A \\
C
\end{array}\right]\right)=n, \quad \forall \lambda \notin \mathcal{D}
$$

\subsection{CONCEITOS BÁSICOS DAS REGIÕES LMI}

As regiões LMI descrevem regiões convexas no plano complexo que são simétricas em relação ao eixo real.

Dentição 3.1. Um subconjunto $D$

$$
\mathcal{D}=\left\{\lambda \in \mathcal{C}: f_{\mathcal{D}}(\lambda)<0\right\}
$$

Plano complexo é chamado de região LMI se houver uma matriz simétrica $\Delta=$ $(\delta)_{k l} \in \Re^{n \times n}$ e a matriz $\Theta=(\theta)_{k l} \in \Re^{n \times n}$ tal que

$$
f_{\mathcal{D}}(\lambda)=\Delta+\lambda \Theta+\bar{\lambda} \Theta^{\prime}
$$

Com $\lambda, \bar{\lambda} \in \mathcal{C}_{\text {onde }} \mathrm{C}$ denota o semiplano complexo ${ }^{1}$ 
A função característica $f_{\mathcal{D}}(\lambda)$ assume valores no espaço de matrizes Hermitianas de dimensões $n \times n$ As desigualdades de matrizes lineares, $(L M I s)_{\text {podem ser definidas a partir de }} f_{\mathcal{D}}(\lambda)$ para caracterizar as propriedades dos autovalores de uma matriz real pertencente a $\mathcal{D}[9]$. As regiões LMI com suas funções características são fornecidas em [9].:

- Um disco-centrico $(-q, 0)$ com raio r, cuja função característica é:

$$
f_{\mathcal{D}}(\lambda)=\left(\begin{array}{rr}
-r & q \\
q & -r
\end{array}\right)+\left(\begin{array}{ll}
0 & 0 \\
1 & 0
\end{array}\right) \lambda+\left(\begin{array}{ll}
0 & 0 \\
1 & 0
\end{array}\right)^{\prime} \bar{\lambda}
$$

Considere o sistema linear representado por

$$
\dot{z}(t)=H z(t), \quad \text { com } H \in \Re^{n \times n}
$$

O sistema (19) é $\mathcal{D}$-estável, onde D é uma região LMI, se e somente se existirem matrizes $\Delta$ e $\Theta \in \Re^{n \times n}$

$$
f_{\mathcal{D}}(\Theta)=\Delta+\Theta \otimes H+\Theta^{\prime} \otimes H^{\prime}<0
$$

onde $\otimes$ representa o produto Kronecker em cite horn91. É interessante notar que:

- a equação (20) pode ser reescrita como:

$$
f_{\mathcal{D}}(\theta)=(\delta)_{k l}+(\theta)_{k l} H+(\theta)_{l k}^{\prime} H^{\prime}, \quad \forall 1 \leq k, l \leq n
$$

- para duas regiões $L M I, \mathcal{D}_{1}$ e $\mathcal{D}_{2}$ e cujas funções características estão associadas a $f_{\mathcal{D}_{1}}$ e $\overline{f_{\mathcal{D}_{2}}}$, respectivamente, a interseção $\mathcal{D}=\mathcal{D}_{1} \cap \mathcal{D}_{2}$ é uma região LMI com função característica $f_{\mathcal{D}_{1} \cap \mathcal{D}_{2}}=\operatorname{Diag}\left(f_{\mathcal{D}_{1}}, f_{\mathcal{D}_{2}}\right)$.

\subsection{D- ESTABILIZAÇÃO POR REALIMENTAÇÃO DE SAÍDA}

Lembre-se primeiro que a existência de uma matriz de realimentação de saída que estabiliza o sistema em malha fechada pode ser associada á solução das equações de Sylvester acopladas (1), (2) e (3). 
Pelo teorema em [5], essas equações podem ser interpretadas em termos de propriedades geométricas do subespaço $\mathcal{V}=\operatorname{Ker}(T)$.

Uma vez encontrada a matriz de realimentação de saída que estabiliza o sistema, o espectro do circuito fechado é dado por:

$$
\sigma(A+B G C)=\sigma\left(H_{V}\right) \dot{\cup} \sigma\left(H_{T}\right)
$$

Assim, na sequência considera-se que a colocação regional desejada dos polos é definida a partir de LMI regiões associadas_as matrizes HV e HT , da seguinte forma:

$$
\begin{aligned}
& \sigma\left(H_{V}\right) \in \mathcal{D}_{V} \\
& \sigma\left(H_{T}\right) \in \mathcal{D}_{T}
\end{aligned} \quad \Longrightarrow \mathcal{D}=\mathcal{D}_{V} \cup \mathcal{D}_{T}
$$

Então, supondo que $H_{V} \in \Re^{p \times p}$ e $H_{T} \in \Re^{n p \times n p}$,D - condições de estabilizabilidade em LMI, as regiões são substituídas de forma equivalente pelas duas condições a seguir ,:

$$
\begin{array}{r}
\sigma\left(H_{V}\right) \in \mathcal{D}_{V} \Longleftrightarrow \exists \Pi=\Pi^{\prime}>0 \text { tal que } \\
\Delta_{V} \otimes \Pi+\Theta_{V}^{\prime} \otimes\left(\Pi H_{V}^{\prime}\right)+\Theta_{V} \otimes\left(H_{V} \Pi\right)=-Q_{V}<0
\end{array}
$$

onde $\Pi \in \Re^{p \times p}$

$$
\begin{array}{r}
\sigma\left(H_{T}\right) \in \mathcal{D}_{T} \Longleftrightarrow \exists \Gamma=\Gamma^{\prime}>0 \text { tal que } \\
\Delta_{T} \otimes \Gamma+\Theta_{T}^{\prime} \otimes\left(H_{T}^{\prime} \Gamma\right)+\Theta_{T} \otimes\left(\Gamma H_{T}\right)=-Q_{T}<0
\end{array}
$$

onde $\Gamma \in \Re^{n-p \times n-p}$.

O seguinte resultado pode ser obtido de forma semelhante ao resultado da seção anterior, considerando como ponto de partida as equações de Sylvester acopladas sob a colocação regional de polos restritas ao espectro das matrizes $\mathrm{H}_{V}$ e $\mathrm{H}_{T}$, respectivamente.

Para fins algorítmicos, considera-se que $v=p$, As condições necessárias e suficientes são apresentadas para a matriz de realimentação de saída de existência dada pelo seguinte teorema.

As condições necessárias e suficientes são apresentadas para a matriz de realimentação de saída de existência dada pelo seguinte teorema.

Teorema 3.1. Existe uma matriz de realimentação de saída estática $G \in \Re^{m \times p}$ tal 
que $\sigma(A+B G C)=\left\{\lambda_{1}, \lambda_{2}, \ldots, \lambda_{n}\right\} \in \mathcal{D}$, for $\mathcal{D}=\mathcal{D}_{V} \cup \mathcal{D}_{T}$, where $\left\{\lambda_{1}, \ldots, \lambda_{n-p}\right\} \in$ $\mathcal{D}_{T}$ and $\left\{\lambda_{n-p+1}, \ldots, \lambda_{n}\right\} \in \mathcal{D}_{V}$, se e somente se existem matrizes $\left(P=P^{\prime} \geq 0\right.$, $P \in \Re^{n \times n}, Y \in \Re^{m \times n}, S=S^{\prime} \geq 0, S \in \Re^{n \times n}$ and $Z \in \Re^{n \times p}$ ) de modo que as seguintes condiçôes sejam verificadas para qualquer par de matrizes $V \in \Re^{n \times p} e$ $T \in \Re^{n-p \times n}$ tal que $T V=0$ :

i)

$$
\begin{gathered}
\Delta_{V} \otimes P+\Theta_{V} \otimes(A P+B Y)+\Theta_{V}^{\prime} \otimes\left(P A^{\prime}+Y^{\prime} B^{\prime}\right)= \\
-\left(V \otimes I_{p}\right) Q_{V 1}\left(V \otimes I_{p}\right)^{\prime} \text { para } Q_{V 1}>0 \\
V^{\prime} P V=\bar{P}>0 ; T P T^{\prime}=0 \\
Y=W_{\Pi} V^{\prime} \text { para alguns } W_{\Pi} \in \Re^{m \times p}
\end{gathered}
$$

ii)

$$
\begin{gathered}
\Delta_{T} \otimes S+\Theta_{T} \otimes(S A+Z C)+\Theta_{T}^{\prime} \otimes\left(A^{\prime} S+C^{\prime} Z^{\prime}\right)= \\
-\left(T^{\prime} \otimes I_{n-p}\right) Q_{T 1}\left(I_{n-p} \otimes T\right) \text { for } Q_{T 1}>0 \\
T S T^{\prime}=\bar{S}>0 ; V^{\prime} S V=0 \\
Z=T^{\prime} U_{\Gamma} \text { para alguns } U_{\Gamma} \in \Re^{m \times p}
\end{gathered}
$$

iii)

$$
\begin{aligned}
\operatorname{Ker} C P & \subseteq \operatorname{Ker} Y \\
\operatorname{Ker} B^{\prime} S & \subseteq \operatorname{Ker} Z^{\prime}
\end{aligned}
$$

\section{Prova:}

Necessidade: Considere que as equações de Sylvester acopladas (1), (2) e (3) são verificados com as condições (4) e (5). Primeiro mostra a parte de necessidade (i).

Uma vez que a condição quadrática dada por (24) deve ser verdadeira, e usando a definição de produto Kronecker ${ }^{2}$

$$
\begin{array}{r}
\left(\delta_{V}\right)_{k l}\left(V \Pi V^{\prime}\right)+\left(\theta_{V}\right)_{l k}\left(V \Pi H_{V}^{\prime} V^{\prime}\right)+\left(\theta_{V}\right)_{k l}\left(V H_{V} \Pi V^{\prime}\right)= \\
-V\left(Q_{V}\right)_{k l} V^{\prime} \forall 1 \leq k, l \leq n
\end{array}
$$

onde $:\left(Q_{V}\right)_{k l}=\left(Q_{V}\right)_{k l}^{\prime} \in \Re^{p \times p}$.

De (1), também é obtido $A V+B W=V H_{V}$,que pode ser usado em (31) obter 


$$
\begin{array}{r}
\left(\delta_{V}\right)_{k l}\left(V \Pi V^{\prime}\right)+\left(\theta_{V}\right)_{l k}\left(V \Pi V^{\prime} A^{\prime}+V \Pi W^{\prime} B^{\prime}\right)+ \\
\left(\theta_{V}\right)_{k l}\left(A V \Pi V^{\prime}+B W \Pi V^{\prime}\right)=-V\left(Q_{V}\right)_{k l} V^{\prime}
\end{array}
$$

Assim, ao colocar as matrizes $P=P^{\prime}=V \Pi V^{\prime}$ e $Y=W \Pi V^{\prime}$,e considerando que o posto $(V)=p$ e que $\Pi>0 \Longrightarrow\left\{\begin{array}{l}V^{\prime} V \Pi V^{\prime} V>0 \\ T^{\prime} V \Pi V^{\prime} T=0\end{array}\right.$, então (32) pode ser substituído de forma equivalente por (24), (24) e (25).

Usando argumentos semelhantes, mostra a parte de necessidade (ii). Assim, de (2) e (24), é obtido ,:

$$
\begin{array}{r}
\left(\delta_{T}\right)_{k l}\left(T^{\prime} \Gamma T\right)+\left(\theta_{T}\right)_{l k}\left(T^{\prime} H_{T}^{\prime} \Gamma T\right)+\left(\theta_{T}\right)_{k l}\left(T^{\prime} \Gamma H_{T} T\right)= \\
-T^{\prime}\left(Q_{T}\right)_{k l} T \forall 1 \leq k, l \leq n
\end{array}
$$

onde: $\left(Q_{T}\right)_{k l}=\left(Q_{T}\right)_{k l}^{\prime} \in \Re^{n-p \times n-p}$.

Para as matrizes $S=T^{\prime} \Gamma T$ e $Z=T^{\prime} \Gamma U$ : (33) pode ser substituído por (26) e (27). Além disso, desde posto $(T)=q-p$ e $\Gamma>0$, é obtido $\left\{\begin{array}{l}T T^{\prime} \Gamma T T^{\prime}>0 \\ V^{\prime} T^{\prime} \Gamma T V=0\end{array}\right.$.

Necessidade da parte (iii), (28) e (29) seguir as condições $\operatorname{Ker} C V \subseteq \operatorname{Ker} W$ e $\operatorname{Ker} B^{\prime} T^{\prime} \subseteq \operatorname{Ker} U^{\prime}$,respectivamente, levando em consideração as definições acima de $P, Y, S$ e $Z$.

\section{Subciência :}

Considera-se que as partes (i), (ii) e (iii) são verificadas.

\section{ALGORITMO DE ESTABILIZAÇÃO DE REALIMENTAÇÃO DE SAÍDA}

\subsection{ALGORITMO PRIMAL}

Com base no algoritmo primal proposto na seção anterior, o seguinte procedimento básico é proposto para calcular uma matriz de realimentação de saída estática estabilizadora $D$ no sistema de malha fechada quando a condição $m+p>n$ é verificada : 
Passo 1:

1:1) Encontre uma decomposição

$$
C\left[\begin{array}{ll}
M_{1} & M_{2}
\end{array}\right]=\left[\begin{array}{ll}
C_{1} & 0
\end{array}\right]
$$

Onde: $C_{1} \in \Re^{p \times p}, \operatorname{posto}\left(C_{1}\right)=p$.

Para calcular matrizes $A_{12}, A_{22}$ :

$$
\left[\begin{array}{l}
A_{21} \\
A_{22}
\end{array}\right]=\left[\begin{array}{l}
\bar{M}_{1}^{\prime} \\
\bar{M}_{2}^{\prime}
\end{array}\right] A M_{2} \text { onde }\left[\begin{array}{ll}
M_{1} & M_{2}
\end{array}\right]\left[\begin{array}{l}
\bar{M}_{1}^{\prime} \\
\bar{M}_{2}^{\prime}
\end{array}\right]=I_{n}
$$

1:2) Resolva o LMI para encontrar $S_{21}$ e $S_{22}=S_{22}^{\prime}>0$ :

$$
\begin{array}{r}
\Delta_{T} \otimes\left(S_{22}\right)+\Theta_{T} \otimes\left(S_{22} A_{22}+S_{21} A_{12}\right)+ \\
\Theta_{T}^{\prime} \otimes\left(A_{22}^{\prime} S_{22}+A_{21}^{\prime} S_{21}^{\prime}\right)<0 .
\end{array}
$$

1:3) Calcular

$$
T=\left[\begin{array}{ll}
S_{21} & S_{22}
\end{array}\right]\left[\begin{array}{l}
\bar{M}_{1}^{\prime} \\
\bar{M}_{2}^{\prime}
\end{array}\right] \cdot \operatorname{Se}(A, B, T)
$$

não tem zeros invariantes, vá para a Passo 2; ou então, repita o Passo 1 usando uma nova decomposição para C.

\section{Passo 2:}

2:1) Calcule V como uma base ortogonal de $\operatorname{Ker}(T)$ :

$$
T V=0 \quad \text { com } \quad V^{\prime} V=I_{p}
$$

2:2) Resolva a seguinte equação, sob as restrições (24) e (25), para encontrar P e Y :

$$
\begin{array}{r}
\Delta_{V} \otimes P+\Theta_{V} \otimes(A P+B Y)+\Theta_{V}^{\prime} \otimes\left(P A^{\prime}+Y^{\prime} B^{\prime}\right)= \\
-\left(V \otimes I_{p}\right) Q_{V 1}\left(V \otimes I_{p}\right)^{\prime} \leq 0
\end{array}
$$

Passo 3: A D-estabilizante a matriz de realimentação de saída no sistema de malha fechada correspondente verifica 


\section{$G C P=Y \Longleftrightarrow G C V \bar{P}=W_{\Pi}$, desde que $V^{\prime} V=I_{p}$.}

Comentário 4.1. Observe também que a equação (36) é um $L M I$ nas variáveis $S_{21}$ e $S_{22}$. Então, técnicas de programação convexa pode ser usado para encontrar soluções viáveis em [2]. Como um teste de viabilidade para o passo 2 , você deve verificar se o sistema $(A ; B ; T)$ não tem zeros invariantes que pertençam a $D_{V}$. Em seguida, vá para a próximo passo. As condições são geralmente resolvidas no sentido de que o sistema de matriz $P_{\lambda}=\left[\begin{array}{rr}A-\lambda I_{n} & B \\ T & 0\end{array}\right]$ da dimensão $(n+m) \times(2 n-p)$ deve ter posto de linha completa forall lambda. Então, o passo 2 só é viável com soluções estáveis. Considere o sistema $(A ; B ; C)$ com dados em $[11] ; 4.2$ Exemplos

$$
A=\left[\begin{array}{rrrr}
0 & 1 & 0 & 0 \\
1 & 1 & 0 & 0 \\
-1 & 0 & 0 & 0 \\
0 & 0 & 0 & 0
\end{array}\right] ; B=\left[\begin{array}{ll}
0 & 0 \\
1 & 0 \\
0 & 0 \\
0 & 1
\end{array}\right] ; C=\left[\begin{array}{cccc}
1 & 0 & 0 & 1 \\
0 & 0 & 1 & 0 \\
0 & 0 & 0 & 1
\end{array}\right]
$$

O sistema correspondente (C; A;B) é estabilizável e detectável, e $m+p=5>n$.

Os pólos de malha aberta são: 0:0000; 0:0000; 1:6180;-0:6180.

\section{A) Algoritmo Primal:}

A região LMI DT _e uma banda vertical definida por: $\mathcal{D}_{T}=\{x+j y \in \mathcal{C} ;-5<$ $x<-1\}$. A região LMI Dv é definido a partir da intersecção de setores e um disco crônico definido por: $\left\{x+j y \in \mathcal{C} ; x<-5,|x+j y|<10\right.$ and $\left.\tan 45^{\circ} x<|y|\right\}$ Assim, técnicas de programação convexa são aplicadas para encontrar soluções viáveis para as equações de posicionamento regional de pólos sem requisitos adicionais.

A matriz $\left[\begin{array}{ll}M_{1} & M_{2}\end{array}\right]=\left[\begin{array}{rrrr}-0.7071 & 0.0000 & 0.7071 & 0.0000 \\ 0.0000 & 0.0000 & 0.0000 & 1.0000 \\ 0.0000 & -1.0000 & 0.0000 & 0.0000 \\ -0.7071 & 0.0000 & -0.7071 & 0.0000\end{array}\right]$ encontrado no passo 1.1 leva a: $A_{22}=1.0000 \quad A_{12}=\left[\begin{array}{r}-0.7071 \\ 0.0000 \\ 0.7071\end{array}\right]$. 
Uma solução para o passo 1:2 is:

$$
S_{22}=333.3318, S_{21}=\left[\begin{array}{lll}
0.0000 & 0.0000 & -2080.8778
\end{array}\right],
$$

o que resulta no passo $1 ; 3$ :

$$
T=\left[\begin{array}{llll}
-1471.4028 & 333.3318 & 0.0000 & 1471.4028
\end{array}\right] .
$$

Esta solução não tem zeros invariantes e implica na solução 2:1:

$$
V=\left[\begin{array}{rrr}
0.6982 & 0.0000 & 0.1582 \\
-0.0650 & 0.0000 & 0.9853 \\
0.0000 & 1.0000 & 0.0000 \\
0.7129 & 0.0000 & -0.0650
\end{array}\right]
$$

Uma solução para o passo 2:2 é, então, encontrada:

$$
\begin{gathered}
Y=\left[\begin{array}{rrrr}
10887.2550 & 150744.2000 & 304.1292 & 45036.8680 \\
-4945.7042 & 52669.6190 & -336.9173 & -16877.4870
\end{array}\right] \\
W_{\Pi}=\left[\begin{array}{rrr}
49513.0880 & 304.1292 & -149730.1600 \\
-18910.8790 & -336.9173 & 52208.9740
\end{array}\right]
\end{gathered}
$$

A matriz de realimentação de saída correspondente que estabiliza o sistema de malha fechada é:

$$
G=\left[\begin{array}{rrr}
-218.6662 & 348.0591 & 303.7491 \\
52.7244 & -78.8494 & -75.4133
\end{array}\right]
$$

resultando: $\sigma(A+B G C)=\{-3.4142,-5.7409,-6.2669 \pm 4.6210 j\} \subset \mathcal{D}_{V} \cdot$ Onde o autovalor $-3.4142 \subset \mathcal{D}_{T}$ corresponde ao passo 1. $\triangle$

\section{CONSIDERAÇÕES FINAIS}

Neste artigo, uma abordagem de realimentação de saída e um algoritmo foramapresentados para calcular soluções por meio de técnicas LMI. A solução das equações de Sylvester foi relacionada á solução das equações de Lyapunov que permitiram obter a colocação de pólos nas regiões LMI. Assim, foram apresentadas as condições necessárias e suficientes para a existência da matriz estática de 
feedback de saída. Este artigo também foi proposto um algoritmo de solução numericamente eficiente para as equações de Lyapunov acopladas para determinar a matriz de realimentação de saída. 


\section{REFERÊNCIAS}

[1] Alexandridis A. T. and Paraskevopoulos P. N.(1996), \A New Approach to Eigenstruture Assignment by Output Feedback, IEEE Trans. Automatic. Control ,41, number 7,July, pp. 1046-1050.

[2] Boyd S. P., El Ghaoui L., Feron E. and Balakrishhnan V.(1994), \Linear Matrix Inequalities in System and Control Theory, Philadelphia SIAM.

[3] Byers R., Nash S. G. (1989), \Approaches to robust pole assignment, Int. J. Control , 49 (1), pp.97117.

[4] Castelan E. B., Gomes da Silva V. (2005), \On the solution of a Sylvester equation appering in descriptor Systems control theory, Systems e Control Letters 54, pp. 109-117.

[5] Castelan E. B., Hennet J. C.J. C., Villarreal E. R. LI.(2003), \Quadratic Characterization and Use of Output Stabilizable Subspaces, IEEE Trans. Automatic Control , 48, No. 4, pp. 654-660.

[6] Castelan E. B., Villarreal E. R. LI., Tarbouriech S. (2002), \Quadratic characterization and use of output stabilizable subspaces for descriptor systems," in Proceddings of the 15th Triennial World Congress of IFAC (IFAC'02), Barcelona, pp. 7-12.

[7] Castelan E. B., Hennet J. C. and Villarreal E. R. LI. (2002), \Output Feeedback Design by Coupled Lyapunov-Like Equations, in 15 th IFAC(IFAC'02), Barcelona, pp. 7-12.

[8] Chen C. T.(1999), \Linear System Theory and Design, Holt, Rinehart and Winston.

[9] Chilali M. , Gahinet P. (1996), \H1 Design with Pole Placement Constraints: An LMI Approach, IEEE Trans. Automatic. Control , 41,03, pp.358-367.

[10] Darouach M. (2006), \Solution to Sylvester equation assciated to linear descriptor systems, Systems and Control Letters Vol. 55, Issue 10, October, pp. 835-838.

[11] Fletcher L. R., Kautsky J., Kolka G. K. G and Nichols N. K.(1988), \Eigenstructure Assingment by Output Feedback in Descriptor Systems, IEEE Trans. Automatic. Control, vol. 42, pp.1457-1468.

[12] Horn R. A. and Jhonsons C. R.(1991), \Topics in Matrix Analysis,Cambridge University Press.

[13] Syrmos V. L. and Abdallah C. T. and Dorato P. and Grigoriadis K.(1997), \Static Output FeedbackA Survey, Automatica, Vol. 33, No 2, pp. 125-137.

[14] Syrmos V. L.,Lewis F. L. (1994), \Bilinear Formulation for the Output Feedback Problem in Linear System, IEEE Trans. Automatic Control , 39, No. 2, pp. 410-414.

[15] Syrmos V. L., Lewis F. L.(1993), \Output Feedback Eigenstructure Assignment Using Two Sylvester Equations, IEEE Trans. Automatic Control , 38, No. 3, pp.495-499.

[16] Tits A. L., Yang Y. (1996), \Globally convergent algorithms for robust pole assignment by state feedback, IEEE Trans. Automat. Control , 41 (1) pp.1432- 1452.

[17] Villarreal E. R. Ll., Vargas J. A. R., Hemerly E. M.(2009), \Static Ouput Feedback stabilization using Invariant Subspaces and Sylvester Equations, TEMA - Tend. Mat. Apl. Comput., 10, No. 1, pp. 99-110. 
[18] Yagoubi M. (2010), \Static output feedback control design for descriptor system, Journal of Control Science and Engineering, 1, pp.1-8.

[19] Zhoua L., Lub G. (2009), \Detection and stabilization for discrete-time descriptor systems via a limited capacity, Automatica, 45, No. 10, pp. 2272-2277. 


\section{NOTAS}

\section{Nota1}

$(\delta)_{k l}\left((\theta)_{k l}\right)_{\text {representam os elementos da matriz }} \Delta(\Theta) \forall 1 \leq k, l \leq n$

Nota 2

Observe que (24) pode ser substituído por: $\left(\delta_{V}\right)_{k l} \Pi+\left(\theta_{V}\right)_{l k}\left(\Pi H_{V}^{\prime}\right)+\left(\theta_{V}\right)_{k l}\left(H_{V} \Pi\right)=$ $-\left(Q_{V}\right)_{k l} \forall 1 \leq k, l \leq n$ 


\section{do:}

\section{Capítulo 6}

\section{STRATEGIES AND INFECTIOUS DISEASES}


Abstract: To a contagious disease D, on a homogeneous network P of size NO and whose basic reproduction number $R_{0}>1$, we attribute a unique signature consisting of a quadruple $\left(\nu_{0}, \beta, \rho, \tau\right)$ which determines its threshold of herd immunity, the expansion of the volume of its infected in $P$, its lethality and the fragment of asymptomatic infected. Reconstructing the signature of $D$ from data of benchmarks should enable to determine strategies to either mitigate or eradicate $D$ using strategy functions. We show how to define these strategies in the classical models and more recent models dealing with the SARS CoV2 pandemic. A model, coined SEIPAHQ model, which include asymptomatic and quarantine, is developed to be used together with the strategy functions. This model is closer to the reality of the present pandemic. It is shown that in all models strategy functions does not effect the basic reproduction number and hence no sensitivity analyses is needed to implement strategies. This may lead to the use of Control Theory and Dynamic Programming to determine an optimal strategy function to mitigate the effects of a pandemic in sectors suffering from its effects. Finally, we give a new estimate of the basic reproduction number of the SARS CoV2 outbreak in Wuhan, showing that it was greater than one and use this to estimate the herd immunity threshold. 


\section{INTRODUCTION}

The study of contagious diseases, and how to control them if there is an outbreak, is of interest to mankind, in general, and in agriculture and farming, in particular. This is the reason why models that enables one to predict the outcome of an outbreak or permits how to either mitigate or eradicate such diseases are of extreme importance. Since one can not sample a whole population or because conducting experiments with human beings and animals are subject to strict policies, one of the ways to sidestep these hurdles is the mathematical modeling of these diseases. For big networks of big geographical size, one of the well known models is the SIR Model, an example of bins models.

Other variants of this model are known and are important because not always the hypothesis of the SIR Model holds. Among these are the SIS, SEIR and SIRS models $([1,13,15,18])$.

One of the main questions one tries to answer when modeling a contagious disease $D$ on a homogeneous network of size NO is to predict the growth of the volume of infected, I(t), by D. Since it is more interesting to study a $\mathrm{D}$ with the potential to become an epidemic we suppose that $R_{0}>1$. So we are interested in the endemic equilibrium. This was the starting point of the Inflation Model suggested in [10]. The Inflation Model can be considered as a two bin model stemming from the SIR Model. There, we took a different approach arguing that, in this case, the volume of infected on the network satisfies a linear differential equation. The outcome of this was a pair $\left(v_{0}, b\right)$, coined the signature of $D$, the first of which being a number in ]0, 1 [ and the latter a distribution. It was shown how to modify the natural threshold of herd immunity of $D$, defined as $l_{\infty}=v_{0} N_{0}$, with a constant external action on $B(t)$, called the inflation function of $D$.

In this paper, we modify the definition of signature, defining the signature of a contagious disease $D$, on a homogenous network $P$ and with $R_{0}>1$, to be the quadruple $\operatorname{sign}(D)=\left(v_{0}, 6, \rho, \tau\right)$ where the first two entrees are defined as before, $\rho$ is the lethality of $D$ and $\tau$ is the fragment of asymptomatic of $I(t)$. The pair $\left(v_{0}, b\right)$ we shall call the partial signature of $D$ and the pair $(\rho, \tau)$ we shall call the pseudo signature of $D$. We generalize the concept of an external action defining the concept of an external action function or strategy function, resulting from a set of actions impacting directly on the inflation

function $b(t)$, as a function defined on $\left[0,+\infty[\right.$, taking values in $] 0,1\left[\right.$, or more generally in $R_{+}$, and whose derivative is left semi-continuous.

Let $\mathrm{D}$ be the set of contagious diseases $\mathrm{D}$ on a homogenous network $\mathrm{P}$. We say that $D_{1}, D_{2} \in \mathcal{D}$ are equivalent if they have the same signature. This defines a equivalent relation on and we denote the 
equivalent classes by $[D]$. We shall still denote by the quotient space. So a contagious disease is a equivalent class which will still be denoted by $D$ instead of $[D]$. We shall also write $D=\operatorname{sign}(D)$, since the signature determines the class of $D$. We turn $D$ into a metric space defining

$$
\|D\|=\left\|\left(\nu_{0}, \beta, \rho, \tau\right)\right\|=\max \left\{\nu_{0},\|\beta\|_{1}, \rho, \tau\right\}
$$

So the distance between $D_{1}=\left(\nu_{01}, \beta_{1}, \rho_{1}, \tau_{1}\right)$ and $D_{2}=\left(\nu_{02}, \beta_{2}, \rho_{2}, \tau_{2}\right)$ is

$$
\left\|D_{1}-D_{2}\right\|=\max \left\{\left|\nu_{01}-\nu_{02}\right|,|| \beta_{1}-\beta_{2} \|_{1},\left|\rho_{1}-\rho_{2}\right|,\left|\tau_{1}-\tau_{2}\right|\right)
$$

We define a partial order on $D$ by

$$
D_{1} \leq D_{2} \text { if and only if } \nu_{01} \leq \nu_{02}, \beta_{1}(t) \leq \beta_{2}(t), \forall t, \rho_{1} \leq \rho_{2}, \tau_{1} \leq \tau_{2}
$$

In short, $D_{1} \leq D_{2}$ if and only if $\operatorname{sign}\left(\left(D_{1}\right) \leq \operatorname{sign}\left(D_{2}\right)\right.$. This permits to compare contagious diseases and compare actions during one epidemic with actions during another epidemic. This might help to be prepared if a contagious disease $D$ suffers mutations. One should expect that the signatures of the first mutations of $D$ are close to that of $D$. We can now state our main theorem which generalizes the main result of $[10]$ and gives us control mechanisms to either eradicate $D$ or mitigate its effects in sectors it effects.

Theorem 1.1 Let $D$ be a contagious disease defined on a homogeneous network $P$, of size $N_{0}$, with signature $\operatorname{sign}^{(D)}=\left(\nu_{0}, \beta, \rho, \tau\right)$. Let $\delta(t)$ be a strategy function induced by a set of actions $A_{\delta}(t)$ whose impact on the inflation function of $D$ is $\delta$ b. Suppose that $\delta^{\prime}(t) \geq \delta_{0} \geq 0, \forall t \in[0,+\infty[$. Then the new immunity threshold satisfies

$$
I_{\delta \infty} \leq \frac{1}{e^{\delta_{0}}} \cdot\left(I_{\infty}\right)^{\|\delta\|_{\infty}}
$$

and the new inflection points $\pi_{\delta}$, say, of the graph of $I_{\delta}(t)$, are the solutions of the equation

$$
\frac{(\delta(t) \beta(t))^{\prime}}{(\delta(t) \beta(t))^{2}}=-1
$$

Moreover if $D_{1} \leq D_{2}$ then 


$$
\begin{aligned}
& \text { 1. } I_{2 \infty}-I_{1 \infty} \leq\left|\nu_{1}-\nu_{2}\right| \cdot N_{0} \\
& \text { 2. } I_{2}(t) \leq e^{\left\|\beta_{1}-\beta_{2}\right\|_{1}} \cdot I_{1}(t) \\
& \text { 3. }\left|\rho_{1} I_{1}(t)-\rho_{2} I_{2}(t)\right| \leq\left(\rho_{1}\left(-1+e^{\left\|\beta_{1}-\beta_{2}\right\|_{1}}\right)+\left|\rho_{1}-\rho_{2}\right|\right) \cdot N_{0} \\
& \text { 4. }\left|\tau_{1} I_{1}(t)-\tau_{2} I_{2}(t)\right| \leq\left(\tau_{1}\left(-1+e^{\left\|\beta_{1}-\beta_{2}\right\|_{1}}\right)+\left|\tau_{1}-\tau_{2}\right|\right) \cdot N_{0}
\end{aligned}
$$

In case we have a decreasing strategy function $\delta$, then

$$
I_{\delta \infty} \leq\left(I_{\infty}\right)^{\delta^{-}} \cdot e^{-\int \delta^{\prime}(s) G(s) d s}
$$

Where

$$
\delta^{-}=\operatorname{Inf}\{\delta(t): t>0\}
$$

The theorem tells us that there is continuous dependance of the signature and gives control over the impact of external action functions on the ination function so that we can conciliate thevarious sectors that are being e_ected during an outbreak of $D$.

Given $D \in \mathcal{D}$, a strategy sequence to eliminate $\mathrm{D}$ is a sequence of functions $\left(F_{n}\right), F_{n}: \mathcal{D} \longrightarrow \mathcal{D}$ such that $F_{n}(D) \longrightarrow(0,0, \rho, \tau)$ A mutation function is a continuous function $F: \mathcal{D} \longrightarrow \mathcal{D}$ and we say that $F(D)$ is a mutation of $D$. Such an $F$ is a local strategy for $D$ if $\left(F^{(n)}\right)$ is a strategy sequence to eliminate D, where $F^{(n)}=F \circ F^{(n-1)}$ ). A global strategy is one that is a local strategy for all $D \in \mathcal{D}$. Our next result guarantees the existence of global strategies, i.e., the existence of strategies which simultaneously mutates each $\mathrm{D}$ to extinction.

Theorem 1.2 Let $\delta$ be a strategy function, as in Theorem 1.1, with $\|\delta\|_{\infty}<1$ and define F : $D \longrightarrow \mathcal{D}$ by $F(\nu, \beta, \rho, \tau)=\left(\frac{I_{\delta \infty}}{N_{0}}, \bar{\delta} \beta, \rho, \tau\right)$ Then $\mathrm{F}$ is a global strategy.

Since the SEIR and other classical models are not suited to deal with the SARS CoV2 pandemic, we propose, in the last section, a model that, together with strategy functions, can be used to have better mechanisms of mitigation. It has as its starting point results from $[2,16]$ and is a nine bin model whose owchart includes bins for quarantine, asymptomatic infected, hospitalized and super spreaders. 


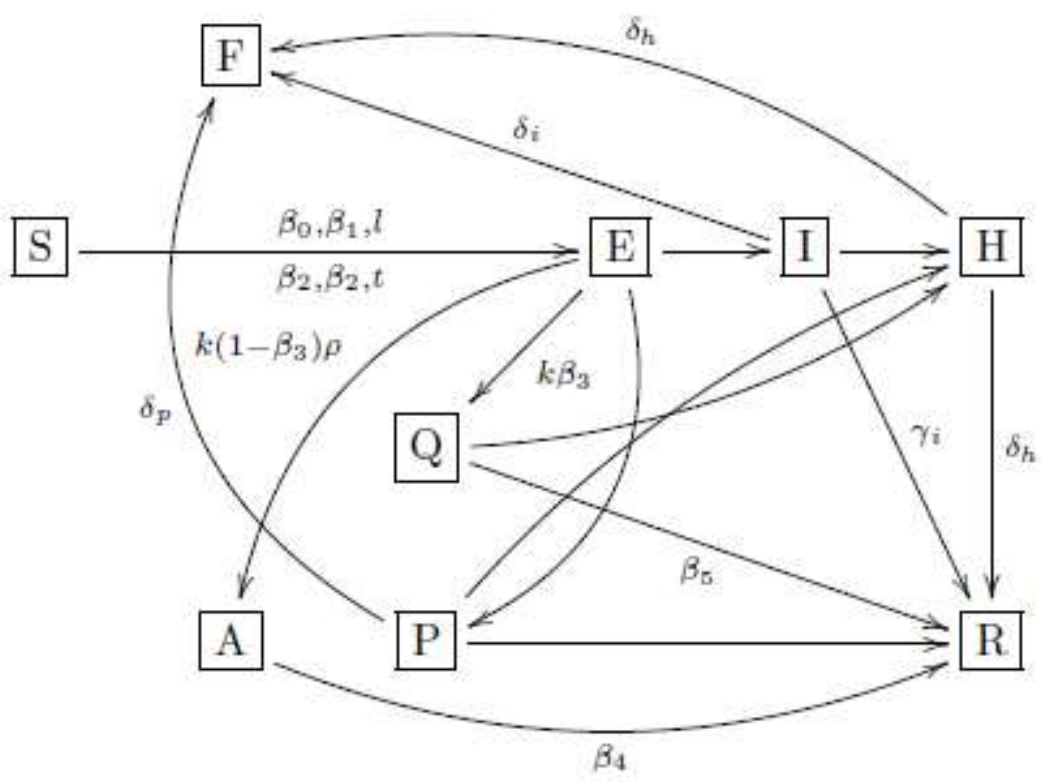

Since our focust is on theoretical development, we do not include applications. These will appear elsewhere. The paper is organized as follows. In the next section, we recall results from [10], define our notion of signature, that of strategy functions, that of strategy and prove our main results. In the third section we generalized results of [10] working also in the SEIR model and a more recent model of SRAS CoV2 given in [2]. In the last section, based on the models and findings in $[2,16]$, we propose a nine bin model to be used together with strategy functions. Using this model, we suggest the correct value of RO in the Wuhan outbreak, correcting its value given in [2]. This leads to an estimate of the herd immunity threshold. Notation is mostly standard and also taken from $[6,10,11]$.

\section{STRATEGY FUNCTIONS OF THE INATION MODEL}

Let $D$ be an outbreak of a contagious disease in a homogeneous network $P$ of size $N_{0}$. In [10] an ination model for $D$ was given which we proceed to describe.

Suppose that herd, or social, immunity is reached when $\nu_{0} \cdot 100 \%$; $] 0,1[$, of $\mathrm{P}$ is infected.

Denote by $\mathrm{I}(\mathrm{t})$ the total number of infected persons and by $\Delta(t)$ the total number of deaths due to infection by $D$ up to that instant. Both are functions of the variable $t$ which is measured in days.

Let $I_{0}=I(0), \Delta_{0}=\Delta(0)$ and $\Delta=\rho$, I where $\left.\rho \in\right] 0,1[$ is a fixed constant (the lethality of P). Up to scaling we may suppose that $I_{0}=1$. The number $I_{\infty}:=\nu_{0} \mathrm{NO}$ is called the natural threshold of herd immunity of $D$. At a fixed instant $t$, each infected contributes to the increase of $I(t)$, this 
contribution may be zero. We denote the sum of these contributions by $\beta(t) I(t)$. If we compare this with, for example, the SIR Model then the recovered up to instant $t$ are included in I(t). We are not looking at the source of the infected but only to the fact that they are inserted in a certain population of finite size, increasing with time, but will never be equal to the whole population. The ination model proposed in [10] supposes that I(t) satisfies a first order linear equation

$$
I^{\prime}(t)=\beta(t) I(t)+f(t)
$$

The function $\beta(t)$ is the ination function of $\mathrm{D}$ and the pair $\left(\nu_{0}, \beta\right)$ was called the signature of $\mathrm{D}$.

Supposing that there is no reinfection by $D$, we have

$$
\begin{aligned}
I^{\prime}(t) & =\beta(t) I(t) \\
\ln \left(I_{\infty}\right) & =\int_{0}^{\infty} \beta(t) d t
\end{aligned}
$$

The fact that $y=I_{\infty}$ is a horizontal asymptote implies that, for $t$ large enough, $\beta^{\prime}(t) \leq 0$, it is an increasing function and $\beta^{\prime}(t) \longrightarrow 0$ as $t \longrightarrow \infty$. Since $I^{\prime \prime}(t)=\left(\beta^{\prime}(t)+\beta^{2}(t)\right) \cdot I(t) I^{\prime \prime}(t)=\left(\beta^{\prime}(t)+\bar{\beta}^{2}(t)\right)$, the inection point $\pi_{0}$, say, of the graph of $\mathrm{I}(\mathrm{t})$, satisfies

$$
\frac{\beta^{\prime}\left(\pi_{0}\right)}{\beta^{2}\left(\pi_{0}\right)}=-1
$$

Now that we have recalled the basics of the Ination Model, we proceed to generalize the concept of external action introduced in [10] and also recall its main theorem.

Definition 2.1 An external action function or a strategy function on $D$ is a function

$$
\delta:[0,+\infty[\longrightarrow[0,1]
$$

whose derivative is left semi-continuous, such that a certain set of external actions on D impact on its ination function as ${ }^{\delta(t)} \beta(t)$. Such a set of actions we shall denote by $A_{\delta}(t)$ so that the dynamics of $D$ becomes

$$
I^{\prime}(t)=\delta(t) \beta(t) I(t)
$$

The resulting solution is denoted by ${ }^{I_{\delta(t)}}$ or $I_{\delta}$ if no confusion arrises. 
In [10] we considered constant external actions and proved the following result.

Theorem 2.2 Let $\mathrm{P}$ be a contagious disease in a population, of size NO, with signature $\left(\nu_{0}, \beta\right)$ and social immunity threshold I1. Let $\infty \delta \in] 0,1\left[\right.$ [ and let $A_{\delta}$ be a set of actions whose impact on the propagation of $\mathrm{P}$ results in the ination function $\delta \cdot \beta$. Then we have.

1. $\nu_{0 \delta}=\frac{\left(\nu_{0}\right)^{\delta}}{\left(N_{0}\right)^{1-6}}$

2. The new social immunity threshold is $I_{\delta \infty}=\left(I_{\infty}\right)^{\delta}$

3. The new inflection points $\pi_{\delta}$ are the solutions of the equation

$$
\frac{\beta^{\prime}\left(\pi_{\delta}\right)}{\beta^{2}\left(\pi_{\delta}\right)}=-\delta
$$

with continuous dependance on $\delta$.

Definition 2.3 Define the signature of a contagious disease $D$, with $\mathrm{RO}>1$, on a homogenous network $\mathrm{P}$ of size No, to be the quadruple sign $(D)=\left(\nu_{0}, \beta, \rho, \tau\right)$ where the first two entrees are defined as before, _ is the lethality of $\mathrm{D}$ and $\_$is the fragment of asymptomatic of $\mathrm{I}(\mathrm{t})$. The pair $\left(\nu_{0}, \beta\right)$ we shall call the partial signature of $D$, denoted by $\operatorname{psign}(D)$, and the pair $(\rho, \tau)$ we shall call the pseudo signature of $D$ and denote it by psdsign(D).

This generalizes the concept of signature introduced in [10]. Since $P$ is homogenous, it should be that the signature of $D$ is an invariant of $D$, i.e., the signature determines $D$. So once we know how $D$ behaves in a homogeneous $\mathrm{P}$ we know how it behaves in any homogeneous $\mathrm{P}$, although there does not exists truly homogeneous networks in reality. This can be of use if $D$ becomes a pandemic. The idea is to reconstruct the signature of $D$ from data of some benchmarks and use it, together with strategy functions, to determine strategies to either mitigating or eradicating $D$.

This can be a protocol to be used to prevent an epidemic to spread elsewhere or to prevent it to become a pandemic. We now prove Theorem 1.1.

\section{PROOF OF THEOREM 1.1}

Proof. Let $G(t)$ be a primitive of $\beta(t)$. Integrating by parts, we that a primitive of $\delta(t) \beta(t)$ satisfies 


$$
\delta(t) G(t)-\int \delta^{\prime}(s) G(s) d s \leq \delta(t) \cdot G(t)-\delta_{0} \cdot \int G(s) d s
$$

Since $I_{\infty}>1$, it follows that there exists $t_{0}>0$ such that $\int_{0}^{t_{0}} G(s) d s>1$ and hence

$$
\delta(t) G(t)-\int \delta^{\prime}(s) G(s) d s \leq\|\delta(t)\|_{\infty} \cdot G(t)-\delta_{0}
$$

From this we get the first estimate. Noting that

$$
I^{\prime \prime}=\left(\delta(t) \beta^{\prime}(t)+\delta^{\prime}(t) \beta(t)+(\delta(t) \beta(t))^{2}\right) I
$$

we obtain the second assertion. Denoting by $\mathrm{G}_{1} ; \mathrm{G}_{2}$ primitives of the respective ination functions, we have that

$$
G_{2}(t)-G_{1}(t) \leq\left\|\beta_{1}-\beta_{2}\right\|_{1}
$$

from which it follows that

$$
I_{2}(t) \cdot\left(I_{1}(t)\right)^{-1} \leq e^{\left\|\beta_{1}-\beta_{2}\right\|_{1}}
$$

and thus

$$
I_{2}(t) \leq e^{\left\|\beta_{1}-\beta_{2}\right\|_{1}} \cdot I_{1}(t)
$$

Using the last inequality, it is easy to prove the rest of the items. Finally, we prove the last statement for a decreasing strategy functions noting that in this case $\delta^{\prime}(t)<0, \forall t>0$. The primitive $F(t)$ of $\delta(t) \beta(t)$ iss $F(t)=\delta(t) G(t)-\int \delta^{\prime}(s) G(s)$ ds and hence

$$
\lim _{t \rightarrow \infty} F(t)=\delta^{-} \cdot \ln \left(I_{\infty}\right)-\int \delta^{\prime}(s) G(s) d s
$$

Hence, it follows that

$$
I_{\delta \infty} \leq\left(I_{\infty}\right)^{\delta^{-}} \cdot e^{-\int \delta^{\prime}(s) G(s) d s}
$$

\section{Proving the Theorem.}

In the last statement of the theorem, one must choose _ to be either a decreasing step function or $\delta$ should first be constant and then $-\delta^{\prime} 0$ should be rapidly decreasing to 0 and designed to minimize the integral in the expression. The theorem is a results about continuous dependency on sign(D) and 
control over the impact of external action functions on the dynamics of D. With a strategy function one can artificially alter the threshold of social immunity while mitigating other sectors.

Given D $2 \mathrm{D}$, a strategy sequence to eliminate $\mathrm{D}$ is a sequence of functions $\left(F_{n}\right), F_{n}: \mathcal{D} \longrightarrow \mathcal{D}$ such that $F_{n}(D) \longrightarrow(0,0, \rho, \tau)$. A mutation function is a continuous function $F: \mathcal{D} \longrightarrow \mathcal{D}$ and we say that $F(D)$ is a mutation of $D$. Such an $F$ is a local strategy for $D$ if $(F(n))$ is a strategy sequence to eliminate $\mathrm{D}$, where $F^{(n)}=F \circ F^{(n-1)}$. A global strategy is one that is a local strategy for all $\mathrm{D} 2 \mathrm{D}$.

\section{WE NOW PROVE THEOREM 1.2.}

\section{Proof of Theorem 1.2}

Proof. We first prove that $\mathrm{F}$ is continuos. Suppose that $\operatorname{sign}\left(D_{n}\right) \longrightarrow \operatorname{sign}(D)$. We shall prove that $F\left(D_{n}\right) \longrightarrow F(D)$. By the definition of $\mathrm{F}$, and the definition of our metric, we just have to prove that psign $p \operatorname{sign}\left(D_{n}\right) \longrightarrow p \operatorname{sign}(D)$, with the obvious meaning. We have that $\left|\int \beta_{n}-\int \beta\right| \leq\left\|\beta_{n}-\beta\right\|_{1}$ and hence

$$
\frac{1}{e^{\left\|\beta_{n}-\beta\right\|_{1}}} \cdot I_{n \infty} \leq I_{\infty} \leq e^{\left\|\beta_{n}-\beta\right\|_{1}} \cdot I_{n \infty}
$$

From this it follows that $I_{n \infty} \longrightarrow I_{\infty}$ and thus $\nu_{0 n}=\frac{I_{n \infty}}{N_{0}} \longrightarrow \frac{I_{\infty}}{N_{0}}=\nu_{0}$. We also have that $\left\|\delta \beta_{n}-\delta \beta\right\|_{1} \leq\|\delta\|_{\infty} \cdot\left\|\beta_{n}-\beta\right\|_{1} \leq\left\|\beta_{n}-\beta\right\|_{1} \longrightarrow 0$. This proves the claim of continuity.

The last part of the proof also shows that $\delta^{n} \beta$, the ination function of $D_{n}=F^{(n)}(D)$ converges to 0 . In fact, $\left\|\delta^{n} \beta\right\|_{1} \leq\|\delta\|_{\infty}^{n} \cdot\|\beta\|_{1} \longrightarrow 0$ since, by hypothesis, $\|\delta\|_{\infty}<1$. Therefore, we are left to prove that $\nu_{0 n} \longrightarrow 0$. In fact, by Theorem 1.1 we have that either

$$
I_{\delta \infty} \leq \frac{1}{e^{\delta_{0}}} \cdot\left(I_{\infty}\right)^{\|\delta\|_{\infty}}
$$

Or

$$
I_{\delta \infty} \leq\left(I_{\infty}\right)^{\delta^{-}} \cdot e^{-\int \delta^{\prime}(s) G(s) d s}
$$

Hence, in both cases, we have that

$$
I_{\delta \infty} \leq 2 \cdot\left(I_{\infty}\right)^{r}, r<1
$$


By induction, we have that

$$
I_{\delta^{n} \infty} \leq\left(I_{\infty}\right)^{r^{n}}<\left(N_{0}\right)^{r^{n}} \longrightarrow 1
$$

From this it follows that

$$
\varlimsup_{n \rightarrow \infty}\left(\nu_{0 n}\right) \leq \frac{1}{N_{0}}
$$

Since we are dealing with a population of big size, we have that $\operatorname{limn} ! \lim _{n \rightarrow \infty}\left(\nu_{0 n}\right)=0$ and the theorem is proved.

\section{STRATEGY FUNCTIONS FOR CLASSICAL MODELS}

In this section we compare the Ination Model, the SIR Model and all other models. We shall be only interested in a contagious disease $\mathrm{D}$ with $\mathrm{RO}>1$ on its way to reaching herd immunity.

Our interest stems from the fact that after this point $D$ can be handled adequately determining whether to eradicate it or whether to let it remain endemic. We recall some classical models that are in use when studying diseases. The scaled SIR model is the System of Non-Linear Diferential Equations

$$
\begin{gathered}
S^{\prime}=-\beta_{0} S I+\mu(N-S) \\
I^{\prime}=\left(\beta_{0} S-\gamma-\mu\right) I \\
R^{\prime}=\gamma I-\mu R
\end{gathered}
$$

Where $R_{0}=\frac{\beta_{0}}{\gamma}$ is the basic reproduction number. In the SIS, SIRS and MSIR models one may also Write $I^{\prime}(t)=\beta(t) I(t)$ Comparing this with the Ination Model, we may use the diferent models to get an expression for $\beta$. For example, in the SIR model we have that $\beta(t)=\gamma \cdot\left(R_{0} \cdot S-1\right)$ This proves that the Ination Model is validated by all these models. Note however that in the Ination Model we work with the total number of infected and so the graph of $\mathrm{I}(\mathrm{t})$ is a logistic curve. In the case of the SIR model, the connection of the ination function $\beta(t)$ and the standard reproduction number $R_{0}$ becomes clear. We choose not to make a network modeling of $D$ (see [18]) but to look how the volume of I(t) grows with time within the network, thus avoiding to make many assumptions. Basically, the only assumption used is that herd immunity is achieved at $I_{\infty}<N_{0}$. In [8] explicit solutions for the SIR Model are given. 
Let us focus on the classical models and de_ne strategy functions in them. These models can be written as $X^{\prime}=F(X)$ Let $\mathrm{D}$ be a contagious disease and denote by $T_{\infty}>0$ the _nite time it takes to reached herd immunity in a homogeneous network $\mathrm{P}$ of size $N_{0}$. We may change the limit $t \longrightarrow \infty$ to $t \longrightarrow T_{\infty}$.

Defnition 3.1 The extended signature of a contagious disease $\mathrm{D}$, with $R_{0}>1$, on a homogenous network $\mathrm{P}$ of size ${ }^{N_{0}}$.is the quintuple sign $(D)=\left(\nu_{0}, \beta, \rho, \tau, T_{\infty}\right)$, where $\left(\nu_{0}, \beta, \rho, \tau\right)$ is the signature of $D$.

De_nition 3.2 An external action function or a strategy function on $\mathrm{D}$ is a function $\delta$ : $\left[0,+\infty\left[\longrightarrow \mathbb{R}_{+}\right.\right.$whose derivative is left semi-continuous, such that a certain set of external ac- tions on $\mathrm{D}$ impact on its ination function as $\delta(t) \beta(t)$. Such a set of actions, denoted by $A_{\delta}(t)$ is such that if the dynamics of $D$ is governed by $X 0=F(X)$ then it is modi_ed such that it is governed by the by $X^{\prime}=F(X)$ modified system $X^{\prime}=\delta(t) F(X)$. The resulting solution of the equation of $\mathrm{I}(\mathrm{t})$ is denoted by $J_{\delta(t)}$, or $J_{\delta}$ if no confusion arrises.

For example, in the SIR model we have $\beta(t)=\beta_{0} S-\gamma-\mu$ and the $\delta$-modified system is

$$
\begin{gathered}
S^{\prime}=-\beta_{0} \delta(t) S I+\mu \delta(t)(N-S) \\
I^{\prime}=\delta(t)\left(\beta_{0} S-\gamma-\mu\right) I \\
R^{\prime}=\delta(t) \gamma I-\delta(t) \mu R
\end{gathered}
$$

Note that an action on the dynamics can be de_ned in other ways but we choose this one because it preserves the phase space of the original dynamics, as we shall see. This action has the property that the basic reproduction number of this system remains the same as the non-modified system. In the Ination Model, we compared the herd immunity threshold of the solutions of both the system and its $\delta$-modification. In our next result we compare the resulting solutions of these two O.D.E.s in the SIR Model showing how they are are linked. The same proof holds for all other models mentioned in this section. Recall that the solution of the Ination Model, after applying an action function $\delta(t)$, is denotes by $I_{\delta}(t)$. 
Theorem 3.3 Let $\delta(t)$ be an external action function. The solution $J_{\delta(t)}$ is induced by a solution $\left(S_{\delta}, J_{\delta}, R_{\delta}\right)$ of the SIR model and we have that $J_{\delta}(t)=I(\hat{\delta}(t))$, where $I(t)$ is the solution in the Ination Model of the $I^{\prime}(t)=\beta(t) I(t)$, with $\beta(t)=\beta_{0} S(t)-\gamma-\mu$ and initial value $J_{\delta}(0)$

Proof. Consider the $\delta$-modified system given above and let $\hat{\delta}(t)$ be a primitive of $\delta(t)$ such that $\hat{\delta}(0)=0$. Let $X_{1}=\left(S_{1}, I_{1}, R_{1}\right)$ be a the solution of the non-modi_ed system, i.e. $\delta=1$ and define $S(t)=S_{\delta}(t)=S_{1}(\hat{\delta}(t)), I(t)=J_{\delta}(t)=I_{1}(\hat{\delta}(t)), R(t)=R_{\delta}(t)=R_{1}(\hat{\delta}(t))$, , i.e., $X(t)=$ $X_{1}(\hat{\delta}(t))$. Since $\hat{\delta}(0)=0$, the initial values of the solution $(S(t), I(t), R(t))$ is $\left(S_{1}(0), I_{1}(0), R_{1}(0)\right.$ and $\left(X_{1}(\hat{\delta}(t))\right)^{\prime}=\delta(t) X_{1}^{\prime}(\hat{\delta}(t))=\delta(t)=F\left(X_{1}(\hat{\delta}(t))=\delta(t) F(X(t)\right.$, we have the solutions are the same. The last statement follows since $\left(I_{\delta}(\hat{\delta}(t))\right)^{\prime}=\delta(t) \cdot I_{\delta}^{\prime}(\hat{\delta}(t))=\delta(t) \cdot \beta(\hat{\delta}(t)) I(\hat{\delta}(t))=J_{\delta}^{\prime}(t)$.

Let $\chi_{n}$ be the characteristic function of the interval $] n, n+1[$. Given a convergent sequence $\left(\alpha_{n}\right)$ in $\mathbb{R}_{+}$we define the associated normalized strategy function as

$$
\chi(t)=\frac{1}{T_{\infty}} \cdot \sum_{n=0}^{\infty} \alpha_{n} \cdot \chi_{n}(t)
$$

It is clear that

$$
\lim _{t \rightarrow \infty} \hat{\chi}(t)=\lim _{n \rightarrow \infty} \alpha_{n}
$$

We are interested in these strategy functions because they are locally constant and thus are suitable to represent actions occurring during a certain period. Recall that these actions impact directly on the ination function. Observe that a strategy function induces a solution of the system whose time we control thus enabling to predict the growth of the ination function. This de_nes a mechanism of control that can be useful in the development of strategies to control the disease. Note that, in the SIR model, the basic reproduction number and the phase space remains unchanged but we control the time of each solution. This means that we still have local stability and that sensitivity analysis is the same as in the unmodified model.

Corollary 3.4 Let $\delta(t)=\chi(t)$ be a normalized strategy function such that 


$$
\left.\lim _{t \rightarrow \infty} \hat{\chi}(t)=\alpha \cdot T_{\infty}, \alpha \in\right] 0,1[
$$

and let $J_{\delta}$ and $\mathrm{l}(\mathrm{t})$ be as in Theorem 3.3. Then

$$
\lim _{t \rightarrow \infty} J_{\delta}(t)=I\left(\alpha \cdot T_{\infty}\right)
$$

and the inection $\pi_{\delta}$ points are given by

$$
\frac{\beta^{\prime}\left(\hat{\delta}\left(\pi_{\delta}\right)\right)}{\beta^{2}\left(\hat{\delta}\left(\pi_{\delta}\right)\right)}=-\delta\left(\pi_{\delta}\right)
$$

The corollary tells us that we can artificially reach herd immunity at $I\left(\alpha \cdot T_{\infty}\right)$ if we use an adequate strategy function. This is the same as was done in the Ination Model. So although we see no change in the phase space $(S ; \mathrm{I})$, when we look at the evolution of $J_{\delta}(t)$ we see it becoming a logistic curve with horizontal asymptote the line $y=I\left(\alpha \cdot T_{\infty}\right)$. Recalling the corresponding herd immunity threshold for the same strategy function in the Ination Model we have that this herd immunity threshold is $I_{\delta \infty}=I_{\infty}^{\alpha}$ but it is not clear if.

$$
I_{\infty}^{\alpha}=I\left(\alpha \cdot T_{\infty}\right)
$$

However, the the idea of artificially controlling the herd immunity level to guide $D$ to attain its natural herd immunity level is the same in both models. We want control either to eradicate or to mitigate D. In [10] we considered that $I^{\prime}(t)=\beta(t) I(t)+f(t)$ but focused on the case when $f=0$. Considering this equation for the Ination Model we can also cover the SEIS, SEIR, MSEIR and MSEIRS models. In all these cases we can de_ne strategy functions and prove results similar to Theorem 3.3 and its corollary, meaning that, also in these cases, we can use strategy functions to control how $D$ reaches its natural threshold of herd immunity. In particular, we look at the SEIR model and a more resent model proposed in [2]. The SEIR model is given by the system

$$
\begin{aligned}
& S^{\prime}(t)=\mu-\mu S-\beta_{0} I S \\
& E^{\prime}(t)=\beta_{0} I S-(\mu+a) E
\end{aligned}
$$




$$
\begin{aligned}
& I^{\prime}(t)=a E-(\gamma+\mu) I \\
& R^{\prime}(t)=\gamma I-\mu R
\end{aligned}
$$

If we apply a strategy function $\delta$ and consider the $\delta$-modified system then, as before, we look at a solution of this system whose infected we denote by $J_{\delta}(t)$. A similar argument gives that $J_{\delta}(t)=I(\hat{\delta}(t))$, where $I(t)$ is the solution of the unmodified system. In this case we have that $R_{0}=\frac{a}{\mu+a} \cdot \frac{\beta_{0}}{\mu+\gamma}$ showing that also in this case Ro remains unmodi_ed and, once more, no sensibility analysis is needed. Actually in both former cases the expression of $R(t)=R_{0} \cdot E(t)$ becomes a function of $\hat{\delta}(t)$ which is continuous.

The model, proposed in [2] for the present SARS CoV2 pandemic is a very general model and, as we shall see in the next section, is closer to reality. The model proposed is given by a system of eight differential equations

$$
\begin{aligned}
& S^{\prime}(t)=-\beta_{0} I S-l \beta_{0} H S-\beta_{1} P S \\
& E^{\prime}(t)=\beta_{0} I S+l \beta_{0} H S+\beta_{1} P S-k E \\
& I^{\prime}(t)=k \rho_{1} E-\left(\gamma_{a}+\gamma_{i}\right) I-\delta_{i} I \\
& P^{\prime}(t)=k \rho_{2} E-\left(\gamma_{a}+\gamma_{i}\right) P-\delta_{p} P \\
& A^{\prime}(t)=k\left(1-\rho_{1}-\rho_{2}\right) E \\
& H^{\prime}(t)=\gamma_{a}(I+P)-\gamma_{r} H-\delta_{h} H \\
& R^{\prime}(t)=\gamma_{i}(I+P)+\gamma_{r} H \\
& F^{\prime}(t)=\delta_{i} I+\delta_{p} P+\delta_{h} H
\end{aligned}
$$

and the constant total population of size $\mathrm{NO}$ is subdivided into eight epidemiological classes: susceptible class $(S)$, exposed class $(E)$, symptomatic and infectious class (I), super-spreaders class (P), infectious but asymptomatic class $(A)$, hospitalized $(H)$, recovery class $(R)$, and fatality class $(F)$. A qualitative analysis is made, $\mathrm{RO}$ is explicited and it is proved that there is a disease-free-equilibrium and an endemic equilibrium, depending on whether $R_{0}<1$ or $R_{0}>1$. The sensitivity analysis for the endemic threshold $\mathrm{RO}$ is also made and the relevant parameters of the model are determined.

They are $\beta_{0}, \rho_{1}$ and $\gamma_{i}$. Our construction applies and we analyze what happens to RO in this case. From [2] we have that 


$$
\begin{gathered}
R_{0}=\frac{\beta_{0} \rho_{1}\left(\gamma_{a} l+\varpi_{h}\right)}{\varpi_{i} \varpi_{h}}+\frac{\left(\beta_{0} \gamma_{a} l+\beta_{0}^{\prime} \varpi_{h}\right) \rho_{2}}{\varpi_{p} \varpi_{h}} \\
\varpi_{i}=\gamma_{a}+\gamma_{i}+\delta_{i}, \varpi_{p}=\gamma_{a}+\gamma_{i}+\delta_{p} \text { and } \varpi_{h}=\gamma_{r}+\delta_{h} \text { Hence, the basic reproduction }
\end{gathered}
$$
number for the $\delta$-modifed model becomes $\dot{R}_{\delta 0}=R_{0}$. Consequently, our strategy method does not effect the basic reproduction number in all models mentioned in this section but effects the growth of the infected population. Note that in general $R_{\delta}(t)=R(\delta(t))$.

Denote by $\mathcal{A}$ the set of action functions (also called admissible controls in Control Theory) and let $G(X, u)=u \cdot F(X), u \in \mathbb{R}$. So given the O.D.E. $X^{\prime}=F(X)$ and $\delta \in \mathcal{A}_{\text {we look the }}$ O.D.E. $X^{\prime}(t)=G(X, \delta(t))$ If we introduce the cost of each sector effected by the pandemic, we can use Dynamical Programming to search for an optional strategy function. Moreover, dividing the population in batches of size at most $10^{5}$, we obtain a collection of systems, coming from the batches, which can be optimally managed as a whole but attending to particular properties of each batch.

\section{THE SEIPAHQ MODEL}

In this section, we revisit the last model in the previous section with the aim to obtain a mechanism of control of the basic reproduction number thus showing the effect of an action function in he Ination Model. Contrary as supposed in [2], we do suppose that asymptomatic persons are infectious and we also include a bin for quarantine, understood in a very general way. That asymptomatic infected are infectious is confirmed by [16] where it it also shown that, however, they are less infectious. But since they accounted of around $90 \%$ of the infected population (see $[5,16])$ they contribute more to the growth of this population. Here quarantine is something very general and include mask wearing, maintaining infected persons in pre-reserved places etc. etc. In the last case, this would make their infectious rate drop close to zero and hence impact directly on the dynamics. We start giving the owchart of our model with some of the parameters. The rest of the parameters can be readout easily from the system and the owchart. 


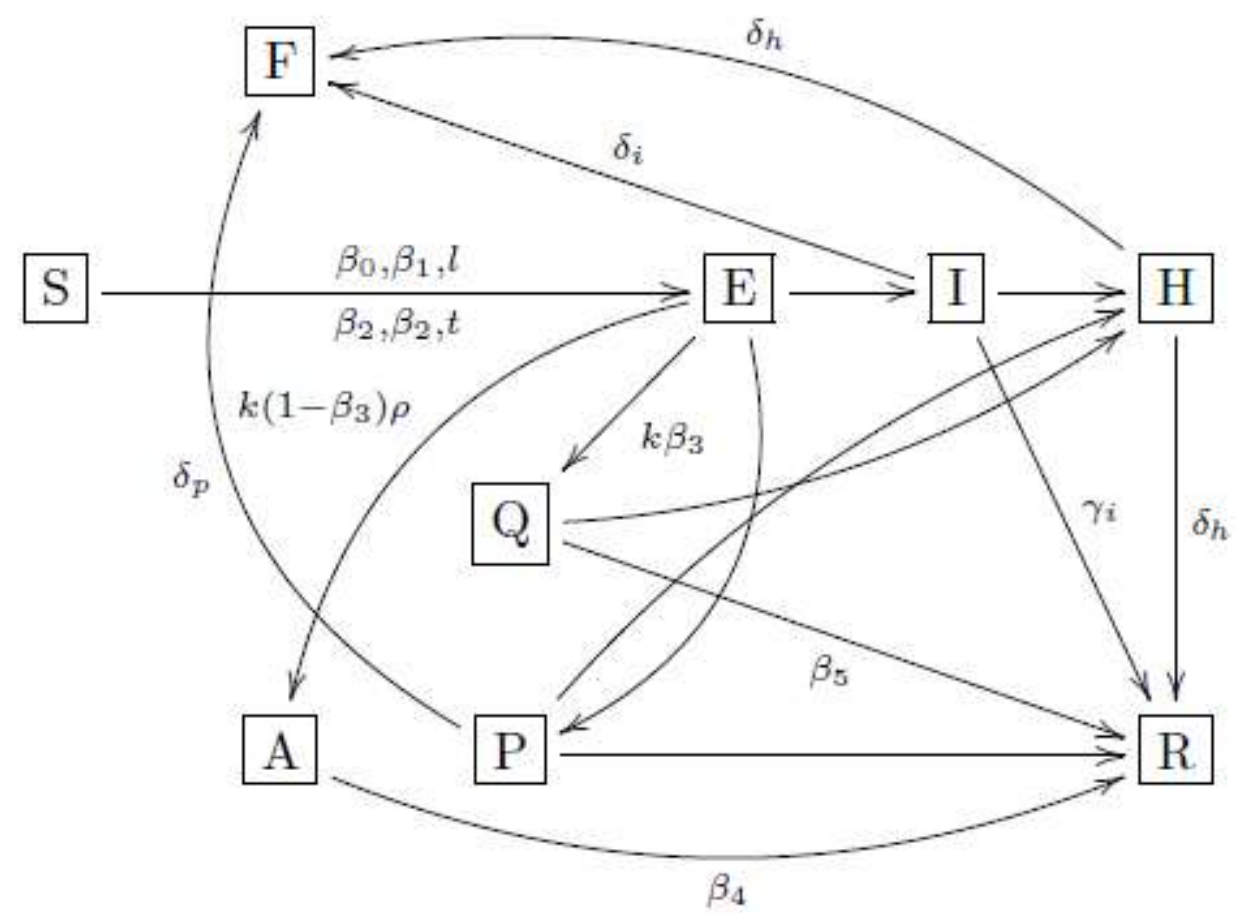

The constant total population, of scaled size $N_{0}=1$ is subdivided into nine epidemiological classes: susceptible class (S), exposed class (E), symptomatic and infectious class (I), superspreaders class (P), infectious but asymptomatic class $(A)$, hospitalized $(H)$, quarantine class $(Q)$, recovery class $(R)$ and fatality class (F). The owchart results in the following nonlinear system of differential equations.

$$
\begin{aligned}
& S^{\prime}(t)=-\beta_{0} I S-\beta_{1} P S-\beta_{2} A S-l H S-q Q S \\
& E^{\prime}(t)=\beta_{0} I S+\beta_{1} P S+\beta_{2} A S+l H S+q Q S-k E \\
& I^{\prime}(t)=k\left(1-\beta_{3}\right)(1-\rho) \rho_{1} E-\left(\gamma_{a}+\gamma_{i}\right) I-\delta_{i} I \\
& P^{\prime}(t)=k\left(1-\beta_{3}\right)(1-\rho) \rho_{2} E-\left(\gamma_{a}+\gamma_{i}\right) P-\delta_{p} P \\
& A^{\prime}(t)=k\left(1-\beta_{3}\right) \rho E-\beta_{4} A \\
& H^{\prime}(t)=\gamma_{a}(I+P+Q)-\gamma_{r} H-\delta_{h} H \\
& Q^{\prime}(t)=k \beta_{3} E-\left(\gamma_{a}+\beta_{5}\right) Q \\
& R^{\prime}(t)=\gamma_{i}(I+P)+\gamma_{r} H+\beta_{5} Q+\beta_{4} A \\
& F^{\prime}(t)=\delta_{i} I+\delta_{p} P+\delta_{h} H \\
& S(t)+E(t)+I(t)+P(t)+A(t)+H(t)+Q(t)+R(t)+F(t)=1
\end{aligned}
$$

To calculate the basic reproduction number we use a result of [6] which also guarantees the existence of a disease free equilibrium if $R_{0}<1$. First we calculate the next generation matrix which is the division of the matrices associated to the rate of appearance of new infections and the net rate out of the corresponding compartments, respectively, 


$$
\begin{gathered}
J_{F}=\left(\begin{array}{cccccc}
0 & \beta_{0} & \beta_{1} & \beta_{2} & l & q \\
0 & 0 & 0 & 0 & 0 & 0 \\
0 & 0 & 0 & 0 & 0 & 0 \\
0 & 0 & 0 & 0 & 0 & 0 \\
0 & 0 & 0 & 0 & 0 & 0 \\
0 & 0 & 0 & 0 & 0 & 0
\end{array}\right) \\
J_{V}=\left(\begin{array}{cccccc} 
\\
-\left(1-\beta_{3}\right) \rho_{1} k(1-\rho) \\
-\left(1-\beta_{3}\right) \rho_{2} k(1-\rho) \\
-\left(1-\beta_{3}\right) k \rho
\end{array} \quad \gamma_{a}+\gamma_{i}+\delta_{i}\right. \\
0
\end{gathered}
$$

The the basic reproduction number is the spectral radius of the matrix

$$
\begin{gathered}
J_{F} \cdot J_{V}^{-1}=\left(\begin{array}{ccccc}
a_{11} & *_{1} & *_{2} & *_{3} & *_{4} \\
0 & 0 & 0 & 0 & 0 \\
0 & 0 & 0 & 0 & 0 \\
0 & 0 & 0 & 0 & 0 \\
0 & 0 & 0 & 0 & 0
\end{array}\right) \\
a_{11}=\left(1-\beta_{3}\right) \cdot\left[(1-\rho)\left[\rho_{1} \cdot \frac{\beta_{0} \varpi_{h}+l \gamma_{a}}{\varpi_{h} \varpi_{i}}+\rho_{2} \cdot \frac{\beta_{2} \varpi_{h}+l \gamma_{a}}{\varpi_{h} \varpi_{p}}\right]+\rho \cdot \frac{\beta_{2}}{\beta_{4}}\right]+\beta_{3} \cdot\left(\frac{q \varpi_{h}+l \gamma_{a}}{\varpi_{h} \varpi_{q}}\right)
\end{gathered}
$$

with $\varpi_{i}=\gamma_{a}+\gamma_{i}+\delta_{i}, \varpi_{p}=\gamma_{a}+\gamma_{i}+\delta_{p}, \varpi_{h}=\gamma_{r}+\delta_{h}$ and $\varpi_{q}=\gamma_{a}+\beta_{5}$.

The characteristic polynomial of the matrix $\mathrm{J}_{\mathrm{F}}, J_{V}^{-1}$ is $\lambda^{4}\left(\lambda-a_{11}\right)$, From this we get that the basic reproduction number is

$$
R_{0}=\left(1-\beta_{3}\right) \cdot\left[(1-\rho)\left[\rho_{1} \cdot \frac{\beta_{0} \varpi_{h}+l \gamma_{a}}{\varpi_{h} \varpi_{i}}+\rho_{2} \cdot \frac{\beta_{1} \varpi_{h}+l \gamma_{a}}{\varpi_{h} \varpi_{p}}\right]+\rho \cdot \frac{\beta_{2}}{\beta_{4}}\right]+\beta_{3} \cdot\left(\frac{q \varpi_{h}+l \gamma_{a}}{\varpi_{h} \varpi_{q}}\right)
$$

This shows the important role played by adopting a quarantine of only infected individuals in certain pre-reserved places. The SARS CoV2 pandemic showed that the asymptomatic infected constitute around $90 \%$ of the infected persons and hence are very important in the count of the herd immunity threshold (see $[11,12,16,5])$. So, although they are also responsible for infections, they are the driving force behind the protection of the herd which shows that any model in which they are not build in is, at least, incomplete. The expression also suggests that $\beta_{4}$ should be more closer to 1 and that $\beta_{2}$ should be smaller than $\beta_{0}$. This would make the asymptomatic infected the driving force behind herd 
immunity. So in the SARS CoV2 pandemic, children and teenagers are more protecting than infecting suggesting that a vertical exposition to the disease might not be a bad strategy.

The expression of R0 also shows that much of this pandemic may not yet be well understood. If we suppose that there is no quarantine, i.e., $\beta_{3}=0$, and since we know that $\rho \approx 0.9, \beta_{2} \approx 0.5 \cdot \beta_{0}$ and $\beta_{4} \approx 1$, we conclude that

$$
R_{0} \approx \frac{1}{10} \cdot\left[\rho_{1} \cdot \frac{\beta_{0} \varpi_{h}+l \gamma_{a}}{\varpi_{h} \varpi_{i}}+\rho_{2} \cdot \frac{\beta_{1} \varpi_{h}+l \gamma_{a}}{\varpi_{h} \varpi_{p}}\right]+\frac{9 \beta_{0}}{20}
$$

Showing that the asymptomatic infected contribute with a around $\frac{\beta_{0}}{2}$ to the value of Ro but reduces the contribution of the others by a factor of 10 . So they infect yet protect the herd. They play both the roles of dark matter and a gravitational constant. If there is a quarantine in which infected are segregated, then $\mathrm{RO}$ is yet more reduced. This shows that, in our model, the relevant parameters are those of [2], already mentioned in the previous section $\beta_{2}, \beta_{3 \text { and }} \rho$.

We look at the stability of our model in terms of R0. First we determine the Jacobean matrix of our model relative to the variables $\mathrm{E} ; \mathrm{I} ; \mathrm{P} ; \mathrm{A} ; \mathrm{H}$ and $\mathrm{Q}$, obtaining

$$
J_{M}=\left(\begin{array}{cccccc}
-k & \beta_{0} & \beta_{1} & \beta_{2} & l & q \\
\left(1-\beta_{3}\right) \rho_{1} k(1-\rho) & -\varpi_{i} & 0 & 0 & 0 & 0 \\
\left(1-\beta_{3}\right) \rho_{2} k(1-\rho) & 0 & -\varpi_{p} & 0 & 0 & 0 \\
\left(1-\beta_{3}\right) k \rho & 0 & 0 & -\beta_{4} & 0 & 0 \\
0 & -\gamma_{a} & -\gamma_{a} & 0 & -\varpi_{h} & -\gamma_{a} \\
\beta_{3} k & 0 & 0 & 0 & 0 & -w_{q}
\end{array}\right)
$$

Using row operations, we put $J_{M}$ in row echelon form concluding that $J_{M}=(I d+E) B$, where the matrices $B$ and $E$ are, respectively, 


$$
\begin{gathered}
B=\left(\begin{array}{cccccc}
-k\left[1-R_{0}\right] & 0 & 0 & 0 & 0 & 0 \\
\left(1-\beta_{3}\right) \rho_{1} k(1-\rho) & -\varpi_{i} & 0 & 0 & 0 & 0 \\
\left(1-\beta_{3}\right) \rho_{2} k(1-\rho) & 0 & -\varpi_{p} & 0 & 0 & 0 \\
\left(1-\beta_{3}\right) k \rho & 0 & 0 & -\beta_{4} & 0 & 0 \\
\frac{k \gamma_{a} \beta_{3}}{\varpi_{q}} & -\gamma_{a} & -\gamma_{a} & 0 & -\varpi_{h} & 0 \\
k \beta_{3} & 0 & 0 & 0 & 0 & -\varpi_{q}
\end{array}\right) \\
E=\frac{\beta_{0} \varpi_{h}+\gamma_{a} l}{\varpi_{h} \varpi_{i}} E_{12}+\frac{\beta_{1} \varpi_{h}+\gamma_{a} l}{\varpi_{h} \varpi_{p}} E_{13}+\frac{\beta_{2}}{\beta_{4}} E_{14}+\frac{l}{\varpi_{h}} E_{15}+\frac{q}{\varpi_{q}} E_{16}+\frac{\gamma_{a}}{\varpi_{q}} E_{56}
\end{gathered}
$$

Hence det $\left(J_{M}\right)=\operatorname{det}(B)=k\left[1-R_{0}\right] \varpi_{i} \varpi_{p} \varpi_{h} \varpi_{q} \beta_{4}$ showing that it is negative if $R_{0}>1$ After a small perturbation if necessary, we may suppose that $k\left[1-R_{0}\right], \varpi_{i}, \varpi_{p}, \varpi_{h}, \varpi_{q}$ and $\beta_{4}$ are all distinct and positive. If $\chi(x)$ denote the characteristic polynomial of $\mathrm{J}_{\mathrm{M}}$, then $\chi(0)=\operatorname{det}\left(J_{M}\right)<0$ If $R_{0}>1$ showing the existence of a positive eigenvalue, and hence, that of an endemic equilibrium. If $R_{0}=0$ we have that the matrix $E=0$ and hence $J_{M}=B$ has only negative eigenvalues. Consequently, there exists $\delta>0$, such that if $0 \leq R_{0}<\delta$ then $\mathrm{J}_{\mathrm{M}}$ has six distinct negative eigenvalues proving that the disease free equilibrium of this model is asymptotically stable. Setting $x=\left(x_{1}, x_{2}, x_{3}, x_{4}, x_{5}, x_{6}, x_{7}, x_{8}, x_{9}\right)=(E, I, P, A, H, Q, S, R, F)$, the disease free equilibrium is $(0,0,0,0,0,0,1,0,0)$ and we may write our system as $x^{\prime}=f(x)$ with $f=\left(f_{1}, \cdots, f_{9}\right)$ With this notation, since in [6, Lemma 1] the matrix $J_{4}=0$ in the disease free equilibrium, we proved that conditions $(A 1)-(A 5)$ of [6, Section 2] are, somehow, all satisfied. Applying [6, Theorem 2] and [19, page 37], it follows that for each set of nonnegative initial conditions there exists a unique solution for our system and that $\mathrm{RO}$ is a threshold parameter for the stability of the disease free equilibrium.

Moreover, if $R_{0}<1$ than the disease free equilibrium is asymptotically stable and unstable if $R_{0}>1$. If $R_{0}=1$ then $\lambda=0$ is a simple eigenvalue of $\mathrm{B}$ and, since $J_{M}=(I d+E) B$ it follows that $B$ and $J_{M}$ have the same null eigenvectors. Since $B$ is in row echelon form, we can explicit An eigenvector $v=\left(1, \frac{k\left(1-\beta_{3}\right)(1-\rho) \rho_{1}}{\varpi_{i}}, \frac{k\left(1-\beta_{3}\right)(1-\rho) \rho_{2}}{\varpi_{p}}, \frac{k\left(1-\beta_{3}\right) \rho}{\beta_{4}}, k \gamma\left(\frac{\beta_{3}}{v a r p i_{q}}+\frac{\rho_{1}}{\varpi_{i}}+\frac{\rho_{2}}{\varpi_{p}}\right), \frac{k \beta_{3}}{\varpi_{h}}\right)$. We calculate that $\chi^{\prime}(0)$ is given by the expression 
$k\left(1-R_{0}\right)\left[\varpi_{i} \varpi_{p} \varpi_{h} \beta_{4}+\varpi_{i} \varpi_{p} \varpi_{q} \beta_{4}+\varpi_{i} \varpi_{p} \varpi_{h} \varpi_{q}+\varpi_{i} \varpi_{h} \varpi_{q} \beta_{4}+\varpi_{p} \varpi_{h} \varpi_{q} \beta_{4}\right]+2 P+S+\varpi_{i} \varpi_{p} \varpi_{h} \varpi_{q} \beta_{4}$, where $P=\gamma_{a} l k\left(1-\beta_{3}\right)(1-\rho) \rho_{1}\left(\varpi_{p} \beta_{4}+\varpi_{p} \varpi_{q}+\varpi_{q} \beta_{4}\right)+\gamma_{a} l k\left(1-\beta_{3}\right)(1-\rho) \rho_{2}\left(\varpi_{i} \beta_{4}+\varpi_{i} \varpi_{q}+\right.$ $\left.\varpi_{q} \beta_{4}\right)+\gamma_{a} l k \beta_{3}\left(\varpi_{i} \varpi_{p}+\varpi_{p} \beta_{4}+\varpi_{i} \beta_{4}\right)$ and $S=\beta_{0} k\left(1-\beta_{3}\right)(1-\rho) \rho_{1}\left(\varpi_{p} \varpi_{h} \beta_{4}+\varpi_{p} \varpi_{q} \beta_{4}+\varpi_{p} \varpi_{h} \varpi_{q}+\right.$ $\left.\varpi_{h} \varpi_{q} \beta_{4}\right)+\beta_{1} k\left(1-\beta_{3}\right)(1-\rho) \rho_{2}\left(\varpi_{i} \varpi_{h} \beta_{4}+\varpi_{i} \varpi_{q} \beta_{4}+\varpi_{i} \varpi_{h} \varpi_{q}+\varpi_{h} \varpi_{q} \beta_{4}\right)+\beta_{2} k\left(1-\beta_{3}\right) \rho\left(\varpi_{p} \varpi_{i} \varpi_{h}+\right.$ $\left.\varpi_{p} \varpi_{i} \varpi_{q}+\varpi_{p} \varpi_{h} \varpi_{q}+\varpi_{i} \varpi_{h} \varpi_{q}\right)+q k \beta_{3}\left(\varpi_{p} \varpi_{i} \beta_{4}+\varpi_{p} \varpi_{i} \varpi_{h}+\varpi_{p} \varpi_{h} \beta_{4}+\varpi_{i} \varpi_{h} \beta_{4}\right)$. From this, it is clear that $\chi^{\prime}(0)>0$ if $R_{0}=1$ and thus, $\lambda=0$ is also a simple eigenvalue of $J_{M}$ for $R_{0}=1$. Using the notation of [6, Section 2], we have that $f_{1}=-f_{7}-k x_{1}$ and that only $f_{1}$ and $f_{7}$ are nonlinear. We can now easily calculate that the expression of $a$ in [6, Section 5.1 Lemma 3] is

$a=\frac{v_{1} w_{7}\left(\beta_{0}+\beta_{1}+\beta_{2}+l+q\right)}{n}$ Since $v_{1}=1$ and $w_{i} \geq 0$ it should be that the bifurcation is subcritical.

Sensitivity analysis must be data driven. For each parameter $\theta$ which R0 depends, its sensitivity index is given by $\frac{\partial R_{0}}{\partial \theta} \cdot \frac{\theta}{R_{0}}$ Note that $\mathrm{R}_{0}$ is a linear affine function of $\theta$ if $\theta \notin\left\{\gamma_{a}, \varpi_{i}, \varpi_{p}, \varpi_{\underline{h}}, \varpi_{q}, \beta_{4}\right\}$ Recall also that all the parameters $\tau \in\left\{\varpi_{i}, \varpi_{p}, \varpi_{h}, \varpi_{q}, \beta_{4}\right\}$ depend on $\gamma_{a}$ and thus $\frac{\partial}{\partial \gamma_{a}}\left(\frac{\gamma_{a}}{\tau}\right)=\frac{\tau-\gamma_{a}}{\tau^{2}}$ From this we get the sensitivity index of all parameters of which $\mathrm{R}_{0}$ depends.

We now look at the e_ect of a strategy function on our model. Setting $X=(S, E, I, P, A, H, Q, R, F)$ and $G(X)$ to be given by the righthand side of our system of equations, we may write the system as $X^{\prime}=G(X)_{\text {If }} \delta(t)$ is an action function then the $\delta$-modified system can be written as $X^{\prime}(t)=\delta(t) G(X)$, or explicitly,

$$
\begin{aligned}
& S^{\prime}(t)=-\left(\delta \beta_{0}\right) I S-\left(\delta \beta_{1}\right) P S-\left(\delta \beta_{2}\right) A S-(\delta l) H S \\
& E^{\prime}(t)=\left(\delta \beta_{0}\right) I S+\left(\delta \beta_{1}\right) P S+\left(\delta \beta_{2}\right) A S+(\delta l) H S-(\delta k) E \\
& I^{\prime}(t)=(\delta k)\left(1-\beta_{3}\right) \rho_{1} E-\left(\left(\delta \gamma_{a}\right)+\left(\delta \gamma_{i}\right)\right) I-\left(\delta \delta_{i}\right) I \\
& P^{\prime}(t)=(\delta k)\left(1-\beta_{3}\right) \rho_{2} E-\left(\left(\delta \gamma_{a}\right)+\left(\delta \gamma_{i}\right)\right) P-\left(\delta \delta_{p}\right) P \\
& Q^{\prime}(t)=(\delta k) \beta_{3} E-\left(\delta \beta_{5}\right) Q \\
& A^{\prime}(t)=(\delta k)\left(1-\beta_{3}\right)\left(1-\rho_{1}-\rho_{2}\right) E-\left(\delta \beta_{4}\right) A \\
& H^{\prime}(t)=\left(\delta \gamma_{a}\right)(I+P+Q)-\left(\delta \gamma_{r}\right) H-\left(\delta \delta_{h}\right) H \\
& R^{\prime}(t)=\left(\delta \gamma_{i}\right)(I+P)+\left(\delta \gamma_{r}\right) H+\left(\delta \beta_{5}\right) Q+\left(\delta \beta_{4}\right) A \\
& F^{\prime}(t)=\left(\delta \delta_{i}\right) I+\left(\delta \delta_{p}\right) P+\left(\delta \delta_{h}\right) H \\
& S(t)+E(t)+I(t)+P(t)+A(t)+H(t)+Q(t)+R(t)+F(t)=1
\end{aligned}
$$

From this it follows that $R_{\delta 0}=R_{0}$ and $R_{\delta}(t)=R_{0} E(\hat{\delta}(t))_{\text {Hence an action function does }}$ not effect the basic reproduction number. Note that this defines an action of the set of action functions on the dynamics of G. We can now apply Control Theory to find an optimal strategy function mitigating 
all the sectors effected by the epidemic. First we should determine the cost function and the function giving the terminal cost of each sector. Once this is done, one can apply Dynamic Programming to obtain an optimal strategy function. In [9] we propose to divide the population in batches of up to 105 and optimally control the set of batches. Assumptions and conditions that hold in one batch are not necessary true in other batches. One can use parameter $\beta_{3}$ as an external action to achieve multiple goals where other external actions have lesser effects.

Since $R(t)=R_{0} E(t)$ and remembering the effect of the asymptomatic infected on $R_{0}$, one can maybe deduce why in most countries the curve of infected starts to level after around a certain period of days. The explanation, once again, might be the asymptomatic infected: they reduce quickly $E(t)$ and this, together with also their effect on Ro makes that $R(t) \leq 1$ quicker. Comparing the expression of $R_{0}$ in this section with its expression in [2] gives a clear view of the importance of the asymptomatic infected. More about this at the end of the section.

The basic reproduction number informed in [2] in the Wuhan case is $R_{0}=0.945$. However, using this value and the data in $[2,16]$, our model formula for $R_{0}$ shows that the correct value is near to $\frac{1}{10} \cdot 0.945+9 \cdot \frac{2.55}{20}=1.242$. This shows that the surge was contained by the lockdown. It would be interesting to see what the percentage of infected in Wuhan is today. For the same reason, the value of $R_{0}$ reported in [14] should also be corrected. In NYC, where there was also a lockdown, we showed in [9] that the percentage of infected doubled between April 20th and May 8th. A similar occurrence was noticed in the UK and the USA. This suggests that the asymptomatic infected should play a bigger role in infecting when con_ned. We also found that the percentage of infected in the population of Sweden was unusually low, compared to other European countries. It suggests that the adopted strategy made the di_erence. This raises the question whether asymptomatic infected should or not be con_ned. Our model suggests that estimations, and the calculated basic reproduction number, should not correspond to reality if the SEIR model is used. Non of these classical models give the correct dynamics of the SARS CoV2 pandemic. Caution should be taken when they are used.

Finally, using the herd immunity threshold formula from Smith and Dietz (see $[4,7,17]$ ) and the Wuhan $\mathrm{R}_{0}$ calculated above, we obtain that the herd immunity threshold is $\nu=1-\frac{1}{R_{0}}=$ $1-\frac{1}{1.242}=0.195$. Hence around twenty percent of the population need to be infected for SARS 
CoV2 to be controlled. This is consistent with the observations in the _ndings of $[9,10,11]$. This is the reason why in most places, where there was no strict confinement, we see the appearance of the inection point in the curve of total infected individuals when around ten percent of the population is infected. So our findings suggest that there should be no second wave if around 10 percent of the population is already infected. Since the asymptomatic infected are around ninety percent of the number of infected individuals, it follows that when the officially informed number of infected is around two percent of the population, the epidemic should start to decline gradually. If there is a higher percentage of officially informed infected, then this should be a consequence of confinement strategies applied aiming to control the pandemic. This must have been the case, for example, in NYC where around forty percent of the population is already infected. In NYC there should be no second wave.

Final Remark: This paper was finished and made available online in June 2020. That is the reason why we did not look at how mutations influence the dynamics, although they were considered in the paper. But since, probably, they are responsible for the observed waves, one may apply the theory here developed to them, as explained in the text. Until late November 2020 our findings agreed with data observed in many cities. We did not check data to see if subsequent waves behaved similarly since we expect them to do so if the signatures of the mutations were close to the original signature. The difference in signature should explain the different behaviors of these subsequent waves but strategies goes along the same lines. Stressors are important to consider when looking at mutations since they can effect the signature. 


\section{REFERENCES}

[1] Anderson, R.M., May, R.M., Infectious Diseases of Humans: Dynamics and Control, OUP (1992).

[2] Faiçal Ndairoua,b, Iván Areab, Juan J. Nietoc, Delfim F.M. Torresa, Mathematical modeling of COVID- 19 transmission dynamics with a case study of Wuhan, Chaos, Solitons and Fractals 135 (2020).

[3] Arenas, A., Costa, W., Gómez-Garde nes, J., Gómez, S., Granell, C., Matamalas, J.T., Soriano, D., Steinegger, B., A mathematical model for the spatiotemporal epidemic spreading of COVID19, preprint.

[4] Dietz K., Transmission and control of arbovirus diseases. In: Ludwig D, Cooke KL, eds. Epidemiology. Philadelphia PA: Society for Industrial and Applied Mathematics, 1975: 104-21.

[5] Dissel, van, J., RIVM, the Netherlands, to appear.

[6] Driessche, van den, P., Watmough J., Reproduction numbers and sub-threshold endemic equilibria for compartmental models of disease transmission, Math. Biosci. 2002 Nov; 180(1 -2) : 29- 48.

[7] Fine, P., Eames, K., Heymann, D. L., Herd Immunity: A Rough Guide, Clinical Infectious Diseases, Volume 52 (7) - Apr 1, 2011.

[8] Harko, T., Lobo, F.S.N, Mak, M.K., Exact analytical solutions of the Susceptible-Infected- Recovered (SIR) epidemic model and of the SIR model with equal death and birth rates, arXiv:1403.2160v1, 2014.

[9] Juriaans, S.O., Signature of Contagious Diseases and Global Strategies, RESEARCHGATE. NET, DOI: 10:13140=RG:2:2:29623:14243, May 2020.

[10] Juriaans, S.O., An Ination Model for Contagious Diseases, RESEARCHGATE.NET, DOI: 10:13140=RG:2:2:26895:12968=2; April2020.

[11] Juriaans, S.O., Number of Infected in Pandemics, RESEARCHGATE.NET, DOI: 10:13140=RG:2:2:36807:44969, April 2020.

[12] Juriaans, S.O., Lopes, J.R., Estimating the Number of Infected in Pandemics, preprint, April 2020.

[13] Keeling, M.J., Rohani, P., Modeling Infectious Diseases in Humans and Animals, Princeton (2007).

[14] Kim, L. et al., The Characteristics of Middle Eastern Respiratory Syndrome Coronavirus

Transmission Dynamics in South Korea , Osong Public Health Res. Perspect 2016 7(1), 49-55.

[15] Li, M.Y. (2018), Basic Mathematical Tools and Techniques. In: An Introduction to Mathemat- ical Modeling of Infectious Diseases. Mathematics of Planet Earth, vol 2. Springer, Cham.

[16] Li, R. et al., Substantial undocumented infection facilitates the rapid dissemination of novel coronavirus (SARS-CoV-2), Science, 10:1126/ Science abb 3221(2020).

[17] Smith, C.E.G., Prospects of the control of disease, Proc Roy Soc Med 1970; 63:1181-90. 
[18] Wells, C.R., The impact of psychology on the effectiveness of voluntary vaccination against infectious diseases in networks, Ph.D. Thesis, University of Guelph, 2012.

[19] Wiggins, S., Introduction to Applied Nonlinear Dynamical Systems and Chaos , Springer, Berlin, 1990. 